


\section{Propositions}

1. It is the degree of plasticity rather than being plastic itself that determines plant competitiveness for light.

(this thesis)

2. Variation in plastic responses result from selection on signal intensity and site of signal perception.

(this thesis)

3. The use of computers may have improved data analysis, it has not improved data interpretation.

4. Research is always biased, however, more in the social than in the natural sciences.

5. International diversity prevents tunnel-vision in all research domains.

6. A non-hierarchical relationship between supervisor and $\mathrm{PhD}$-candidate is required to develop new ideas in scientific research.

7. Propositions should be part of the PhD thesis itself and thus printed within, or discarded altogether.

Propositions belonging to the $\mathrm{PhD}$ thesis entitled:

"How Virtual Shade Sheds Light on Plant Plasticity"

Franca J. Bongers

July $4^{\text {th }}, 2017$ 



\section{How Virtual Shade Sheds Light on Plant Plasticity}

Franca J. Bongers 


\section{Thesis committee}

\section{Promotor}

Prof. Dr N.P.R Anten

Professor of Crop Weed Ecology

Wageningen University \& Research

\section{Co-promotors}

Prof. Dr R. Pierik

Professor of Plant Photobiology

Utrecht University

Dr J.B. Evers

Assistant professor, Centre for Crop Systems Analysis

Wageningen University \& Research

\section{Other members}

Prof. Dr B. Zwaan, Wageningen University \& Research

Dr K. Kahlen, Hochschule Geisenheim University, Germany

Dr H. Huber, Radboud University Nijmegen

Dr M.C.G. Proveniers, Utrecht University

This research was conducted under the auspices of the C.T. de Wit Graduate School for Production Ecology and Resource Conservation 


\title{
How Virtual Shade Sheds Light on Plant Plasticity
}

\section{Franca J. Bongers}

\author{
Thesis
}

submitted in fulfilment of the requirements for the degree of doctor at Wageningen University

by the authority of the Rector Magnificus,

Prof. Dr A.P.J. Mol,

in the presence of the

Thesis Committee appointed by the Academic Board

to be defended in public

on Tuesday 4 July 2017

at 1.30 p.m. in the Aula. 
Franca J. Bongers

How virtual shade sheds light on plant plasticity 140 pages.

PhD thesis, Wageningen University, Wageningen, the Netherlands (2017) With references and with summaries in English and Dutch

ISBN: 978-94-6343-204-7

DOI: $10.18174 / 414191$ 


\section{Abstract}

Phenotypic plasticity is the ability of a genotype to express multiple phenotypes in accordance with different environments. Although variation in plasticity has been observed, there is limited knowledge on how this variation results from natural selection. This thesis analyses how variation in the level of plasticity influences light competition between plants and how this variation could result from selection, driven by light competition, in various environments. As an exemplary case of phenotypic plasticity, this thesis focusses on phenotypic responses of the annual rosette plant Arabidopsis thaliana (Brassicaceae) in response to the proximity of neighbour plants, as signalled through the red : far-red (R:FR) ratio, which are responses associated with the shade avoidance syndrome (SAS).

Plant experiments were conducted to measure variation in these plastic responses and a functional-structural plant (FSP) model was created that simulates plant structures in 3D and includes these organ-level plastic responses while simulating explicitly a heterogeneous light environment. Simulating individual plants that explicitly compete for light, while their phenotype changes through plasticity, gave insights in the role of the level of phenotypic plasticity and site of signal perception on plant competitiveness. In addition, an analysis on how natural selection in different environments acts on the level of plasticity was performed by combining FSP simulations and evolutionary game theoretical (EGT) principles.

Keywords: Arabidopsis, competition, functional-structural plant model, game theory, natural selection, phenotypic plasticity, shade avoidance 



\section{Contents}

CHAPTER 1 General introduction

9

CHAPTER 2 From shade avoidance responses to

plant performance at vegetation level:

using virtual plant modelling as a tool

CHAPTER 3 Subtle variation in shade avoidance responses have profound consequences for plant competitiveness

CHAPTER 4

Tissue specific R:FR perception induces

different adaptive plastic responses

CHAPTER 5

Different environments select for variation in plastic responses

CHAPTER 6 General discussion

References

English summary

Nederlandse samenvatting

Acknowledgements

Curriculum vitae

Publications 

CHAPTER 1

General introduction 
The functioning and performance of organisms is determined by the environment as characterized by the climate determined by among others temperature and water availability and by interactions with other organisms. These environmental characteristics can differ between different locations, such as a tropical rainforest as opposed to a desert, but also in time, like through sudden floods, droughts or insect attacks. The foundation of ecological and evolutionary thinking is that organisms have evolved specific traits to deal with a given environment and its fluctuations. These traits can be fixed per organism, but can also change in response to changes in the environmental conditions, a phenomenon known as phenotypic plasticity. The common definition of phenotypic plasticity is the potential for a genotype to produce multiple phenotypes (i.e. traits) across a range of environments (see Glossary; Bradshaw, 1965; Sultan, 2000; DeWitt \& Scheiner, 2004). Phenotypic plasticity is a common phenomenon for both plants and animals, and has ecological benefits in heterogeneous environments: organisms with phenotypic plasticity can express phenotypes that better fit to the changing environment compared to organisms without plasticity (reviewed in van Kleunen \& Fischer, 2005; Nicotra et al., 2010). Especially for plants, phenotypic plasticity can be crucial, as plants are sessile organisms and are therefore extremely limited in the extent to which they can move away from unfavourable or towards favourable conditions. The extent to which plants need to be plastic depends on the variable environment and the cost related to plasticity. Plasticity evolves in highly variable environments and when inducing plasticity has low costs (Via et al., 1995; DeWitt et al., 1998; Ernande \& Dieckmann, 2004). Variation in the level of plasticity also exists (reviewed in Schmitt et al., 2003; Valladares et al., 2006), suggesting that selection for different levels of plasticity occurs. However, it remains unclear how these different levels of plasticity are the result of selection in different environments.

The main objective in this thesis is to determine to what extent the level of plasticity affects competition between organisms and to analyse how this level of plasticity could result from selection in different environmental conditions. To reach this objective several aspects are important to consider. First of all, it is required to measure and describe variation in plasticity and determine the physiological processes underlying these plastic responses and variation therein. Secondly, as plastic responses occur at the organ level, scaling up is needed from organ-level plasticity to whole-plant phenotype and performance 
in various environments. Finally, it is important to consider that the role of organ-level plasticity for plant performance depends on the environment which is created by the presence of other plants. Considering the presence of multiple organisms competing for a common resource determines how natural selection will act on the level of plasticity. In this thesis I consider the above mentioned aspects by combining (physiological) plant experiments, 3D functional-structural plant (FSP) modelling and evolutionary game theoretical (EGT) principles (see Glossary). As a case study I focus on plastic responses exhibited by the model plant Arabidopsis thaliana to neighbour proximity. In this general introduction I will introduce phenotypic plasticity from a physiological, ecological, evolutionary and environmentally dynamic perspective and introduce important concepts that are required to reach the main objective.

\section{Phenotypic plasticity from an ecological perspective}

Phenotypic plasticity is often related to changes in abiotic environmental conditions (reviewed in Sultan, 2000;

\section{Glossary}

Environmental signal: a physical entity, such as a chemical, wavelength or sound, that gives information and can elicit a response (e.g. R:FR)

Environmental cue: environmental signal that has a meaning regarding the environment and is therefore used to identify an environmental condition (e.g. R:FR signal used as cue for neighbour proximity)

Evolutionary game theoretical (EGT) principles: the application of game theory to explain evolving populations of organisms in biology. In general EGT principles test if a monoculture population with a given strategy can be invaded by an initially rare individual with a different strategy.

Evolutionarily stable strategy (ESS): the strategy that individuals of a monoculture population have by which the monoculture cannot be invaded by an initially rare individual with any alternative strategy

Functional-structural plant (FSP) model: a type of model that simulates plants by combining plant architecture (e.g. 3D structure of organs) with physiological processes (e.g. photosynthesis) and an environmental factor (e.g. R:FR ratio)

Phenotypic plasticity: the ability of an individual to express multiple phenotypes in accordance with different environments

(Plastic) response curve: mathematical description of the trait change upon the perception of an environmental signal (see Box 1.1)

Shade avoidance syndrome (SAS): a set of phenotypic responses displayed by plants that are subjected to shade conditions (e.g. stem elongation) 
Valladares et al., 2007; Nicotra et al., 2010). In addition, plasticity is also of great importance for invasive species, in which phenotypic plasticity could stabilize fitness in unfavourable conditions or increase fitness in favourable conditions (reviewed in Richards et al., 2006; Davidson et al., 2011). Phenotypic plasticity is also related to biotic interactions such as competition for resources. For plants this is mostly competition for light, water and nutrients with other plants. As resource availability to a plant is affected by other plants, it is important to be aware of the presence of these neighbour plants. During the development of a vegetation stand, several signals from both the below- and aboveground environment can indicate the presence of other organisms (reviewed in among others Chen et al., 2012; Pierik \& de Wit, 2014): these include nutrient concentrations, root exudates, volatiles and the spectral light composition. Plants should use these signals to induce phenotypic plasticity in order to increase the plants ability to capture the most limiting resource (based on the functional equilibrium reasoning of Brouwer (1963)). For example, the presence of root exudates can induce changes in root formation or increases carbon allocation to the root (Biedrzycki et al., 2010), so uptake of water and nutrients can be improved, optimizing competitiveness for these resources. The change in the ratio between red $(R, \lambda=600-700$ $\mathrm{nm}$ ) and far-red (FR, $\lambda=700-800 \mathrm{~nm}$ ) light is the best established signal for neighbour proximity above ground, and is related to competition for light. When plants are in close proximity (i.e. high population density), leaves and other green organs will mainly absorb $\mathrm{R}$ light and reflect FR light therefore decreasing the R:FR ratio (Morgan \& Smith, 1978; Ballaré et al., 1987). Plants that grow under conditions with competition for light (e.g. grasslands and crop fields) are known to induce phenotypic responses to a decrease in R:FR light, also referred to as the shade avoidance syndrome (SAS see Glossary; Smith \& Whitelam, 1997; Franklin, 2008; Casal, 2012; Ballaré \& Pierik, 2017).

\section{Shade avoidance syndrome (SAS)}

SAS responses are typical phenotypic changes to reduce shading by neighbours by placing the leaves in a better place of the canopy to capture more light. Depending on the species, SAS responses include increased stem, internode and petiole elongation, upward movement of the leaves (hyponasty), reduced branching or tillering and accelerated flowering (Casal et al., 1986; Ballaré et al., 1987; Pierik et al., 2003; de Wit et al., 2012). For example, Arabidopsis thaliana exhibits upward leaf movement and increased petiole elongation when 


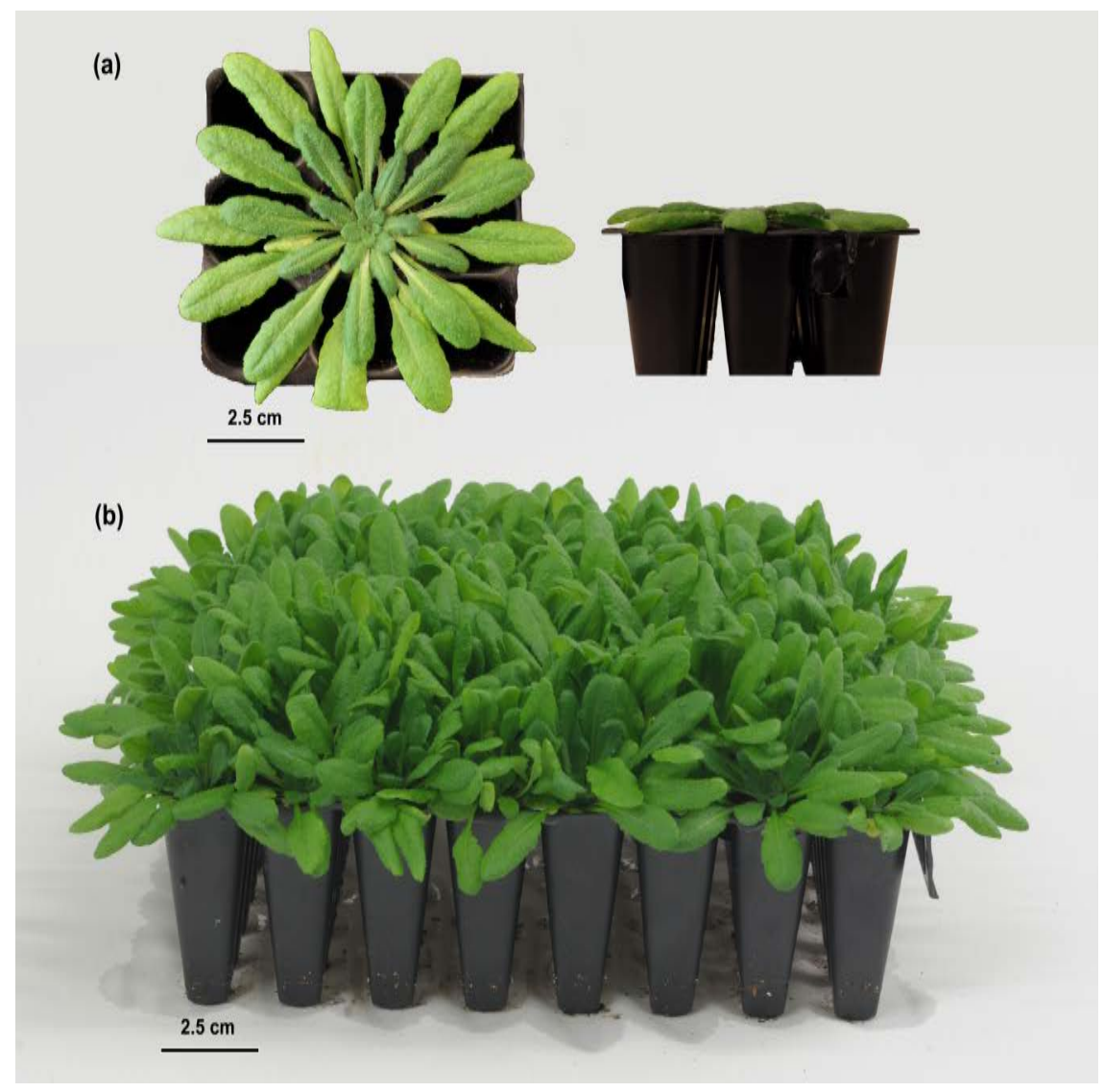

Figure 1.1 Representation of Arabidopsis thaliana plants that grew in a low (a, e.g. 100 plants $\left.\mathrm{m}^{-2}\right)$ or high (b, 1600 plants $\mathrm{m}^{-2}$ ) population density for 46 days. (a) Plants in low density have flat leaf angles and relative short petioles. (b) Plants in high density have elevated leaf angles and longer petioles.

growing in close proximity of neighbours (Figure 1.1). The benefit of inducing these SAS related phenotypes in the high density was proven to depend on the ability to perceive the R:FR ratio: plants that can perceive R:FR changes have long stems in high density and short stems in low density resulting in higher performance than plants with constitutively long or short stems in both high and low density because these latter could not sense R:FR changes (Schmitt et al., 1995; Dudley \& Schmitt, 1996; Ballaré \& Scopel, 1997). Understanding the physiological processes that are responsible for inducing SAS responses in response to the perception of R:FR is outlined in the paragraph Phenotypic plasticity from a physiological perspective.

Although many plant species exhibit these SAS responses, plants from open grasslands have been shown to exhibit stronger shade-induced plastic responses than plants from closed-canopy forests (Schmitt, 1993; Dudley \& Schmitt, 1995; Donohue et al., 2000; 
Anten et al., 2009). This observation of variation in the levels of plasticity in different environments suggests that environments may select for different levels of plasticity (reviewed in Schmitt et al., 2003). In open grasslands where species of similar growth forms compete, low R:FR indicates early neighbour proximity, and inducing SAS responses can therefore put the leaves in a better position of the vegetation to increase light capture. In contrast, in closed-canopy forests low R:FR conditions may imply full shade created by tall trees. This means that inducing SAS responses to low R:FR conditions will not increase light capture. In this ecosystem low R:FR can induce other (plastic) responses that enable plants to deal with shaded conditions. This reasoning implies that selection for the level of phenotypic plasticity depends on the environment; a low R:FR signal is reliable as cue for neighbour proximity in open grasslands but less so in closed-canopy forests. To analyse this kind of environment-dependent selection for variation in phenotypic plasticity, it is required to use a method that regards the variable environment (e.g. R:FR) as consequences of the vegetation itself (i.e. the presence of neighbour plants) and can estimate how selection depends on this environment. The paragraphs Phenotypic plasticity from an evolutionary perspective and Phenotypic plasticity from an environmentally dynamic perspective, will outline the methods that can approach this. In addition, it is required to treat phenotypic plasticity as a quantitative trait, expressing the level of plasticity by variation in a trait value.

\section{Phenotypic plasticity described by a reaction norm}

A common way to express phenotypic plasticity in ecological studies is by a so-called "reaction norm" (Via et al., 1995). In a reaction norm, a set of phenotypes (trait) produced by a genotype are related to a range of environments; a horizontal line illustrates a genotype without plasticity and an increasing or decreasing line illustrates a genotype that is plastic (Figure 1.2a). Different slopes of the reaction norms indicate variation in plasticity (Figure 1.2b), by which the value of this slope can be used as quantitative trait for the level of plasticity. Based on the slope of the curves, genotypes or species are identified as being "more" or "less" plastic (can also be referred to as higher or lower plasticity). If this difference in plasticity is genetic (i.e., different genotypes exhibiting different reaction norms) then this is essentially the same as the genotype $x$ environment ( $G \times E$ ) interaction term (Pigliucci, 2005; Nicotra et al., 2010). In addition, the calculation of plasticity indices has been applied to quantify the level of plasticity (reviewed in Valladares et al., 2006). This 


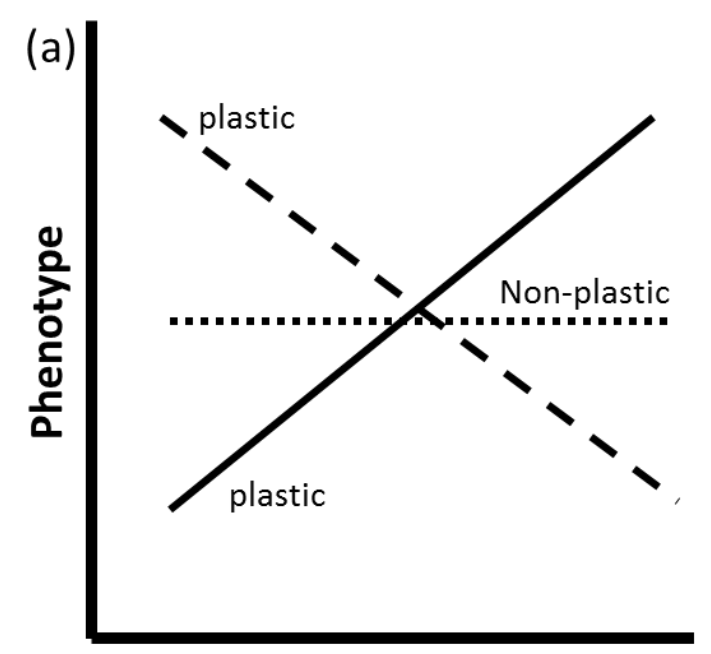

Environment

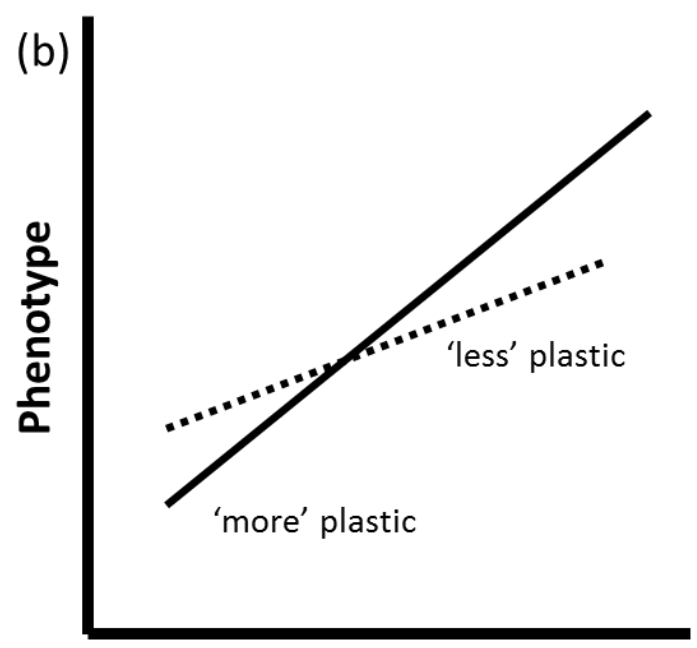

Environment

Figure 1.2 Representation of reaction norms that illustrate phenotypic plasticity. (a) plastic versus nonplastic genotypes. (b) 'more' versus 'less' plastic genotypes. These panels often depict absolute and discrete trait values expressed in different environments. Adapted from (Sultan, 2000; DeWitt \& Scheiner, 2004; van Kleunen \& Fischer, 2005; Pigliucci, 2005; Nicotra et al., 2010).

quantification of the variation in plasticity has been used to explain specific ecological systems such as changes in species composition. For example, studies have concluded that the ability of species to become invasive in new habitats depends on their level of plasticity (Davidson et al., 2011). Although these reaction norms and indices may be useful to express different levels of phenotypic plasticity based on static ecological observations, it is less useful in dynamic eco-physiological studies that consider phenotypic plasticity as plastic responses based on physiological processes that are affected by changing environmental conditions. Therefore, when considering dynamic environmental changes and plant growth explicitly, which will be the case in this thesis, phenotypic plasticity should be expressed by a trait change in response to the perception of an environmental signal instead of the absolute trait value itself. The relationship between these trait changes and a range of environmental conditions will from here on be described by a function that is referred to as a plastic response curve (for further explanation see Box 1.1).

\section{Phenotypic plasticity from a physiological perspective}

Physiological processes are needed to translate the perception of an environmental signal into the induction of a phenotypic response. Extensive molecular and physiological research 


\section{Box 1.1 The difference between a reaction norm and a plastic response curve}

The most common way to express phenotypic plasticity is through a reaction norm that relates the absolute phenotypic trait to variation in the environment (Schmitt, 1993; Dudley \& Schmitt, 1996; Sultan, 2001; Pigliucci et al., 2003; Weijschede et al., 2008); for example long stems in high density and short stems in low density (Figure 1.3a). As it has been established that the ratio of red to far-red (R:FR) light is often low in high density and high in low density (Ballaré et al., 1990), the reaction norm can also express stem length to a continues R:FR environment (Figure 1.3b). These reaction norms describe static trait observations made at a specific moment in time. However, when considering dynamic plant growth, traits should be expressed as change in time (e.g. stem growth rate). Differences in growth rate will eventually determine the final stem length in two environments which are determined by distinct environmental conditions or signals. Here we present a plastic response curve that described plasticity as plastic responses over a range of environmental signals. When the trait change can only express two values, the plastic response can be described by a step-wise response curve (Figure 1.3c). When the trait change can expresses continues values in relation to a continues environment, plastic responses can be expressed by a gradual response curve (Figure $1.3 d)$.
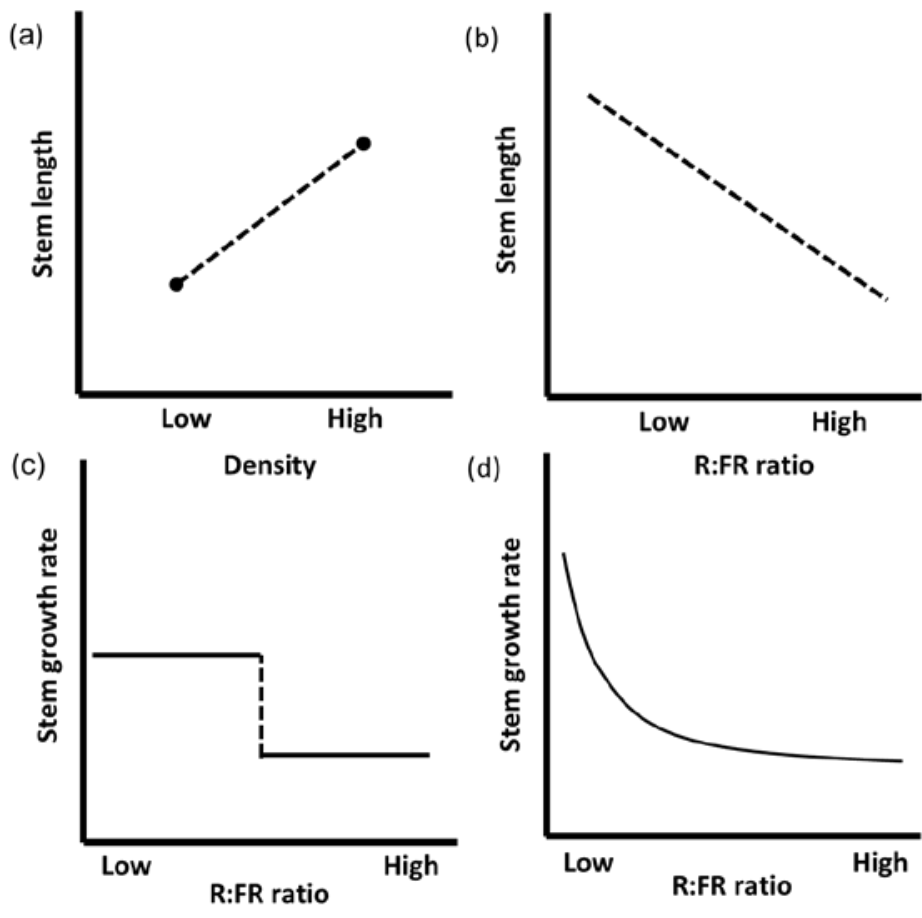

Figure 1.3 The difference between a reaction norm and a plastic response curve. (a-b) Phenotypic plasticity expressed by a classic reaction norm; absolute trait value at a given moment in time related to discrete environments (density (a)) or a continues environment R:FR (b)). (c-d) Phenotypic plasticity expressed by a plastic response curve; trait change related to a continues range of environments, identified by the R:FR ratio. The response curve can be a step-wise (c) or a gradual (d) response curve. 
has been performed on low R:FR-induced SAS responses (reviewed in among others Casal, 2013; Ballaré \& Pierik, 2017). The R:FR ratio is perceived by phytochrome photoreceptors, and inactivating these receptors, as has been done in phytochrome knockout mutants, has been shown to inhibit the ability of plants to induce appropriate phenotypic changes in response to different population densities (among others Schmitt et al., 1995; Ballaré \& Scopel, 1997). Phytochrome photoreceptors exist in an active and inactive isoform (Smith, 2000). As the active form absorbs $F R$ light and the inactive form absorbs $R$ light, the overall activity of these photoreceptors depends on the R:FR environment. In normal sunlight (R:FR ratio around 1.2), a relatively large fraction of active phytochrome is present that triggers among others the inactivation and/or degradation of PHYTOCHROME INTERACTION FACTORSs transcription factors, notably PIF4, PIF5 and PIF7, (PIFs; Duek \& Fankhauser, 2005; Leivar et al., 2008; Leivar \& Quail, 2011). When the R:FR ratio decreases below 1, relatively more inactive phytochrome will be present and the above-mentioned degradation of PIFs is not triggered. If PIFs are not degraded, they can trigger expression of growth-promoting genes (reviewed in Leivar \& Monte, 2014). These include, but are not limited to, genes encoding negative SAS regulators (Sessa et al., 2005; Hornitschek et al., 2009) and genes encoding proteins involved in hormone signalling and biosynthesis, such as auxin (Tao et al., 2008; Li et al., 2012; Hornitschek et al., 2012; de Wit et al., 2015). Altogether, the proteins encoded by these genes interact with again other genes and/or proteins creating a complex signalling pathway that eventually leads to a phenotypic change (reviewed in Casal, 2013; Ballaré \& Pierik, 2017).

Auxin has been demonstrated as key regulator in shade avoidance responses through increased auxin biosynthesis upon low R:FR perception (Tao et al., 2008; Li et al., 2012; de Wit et al., 2015) and decreased phenotypic responses when auxin transport was inhibited (Keuskamp et al., 2010). In addition to auxin, also other hormones such as ethylene, gibberellin and brassinosteroid have been shown to be involved in shade induced responses (reviewed in Casal, 2013; de Wit et al., 2016a; Ballaré \& Pierik, 2017). Knowing the factors involved in the signalling pathway is important when studying selection for phenotypic plasticity because any change within this pathway can result in a different phenotypic response. This means that the use of mutants can be a useful tool to determine variation in plasticity. This variation in plasticity can then be useful to determine the adaptive value of 
signal regulation for plant competitiveness, by letting these mutants compete among each other (Dudley \& Schmitt, 1995; Ballaré \& Scopel, 1997; Pierik et al., 2003; Keuskamp et al., 2010). In addition, knowledge on the factors in the signalling pathway can help optimize plastic responses of specific species to achieve the best plant phenotype to optimize food and flower production.

\section{Phenotypic plasticity from an evolutionary perspective}

Mathematical models have been used to determine how and when plants with phenotypic plasticity are selected for compared to plants with no plasticity. It is theorized that selection for phenotypic plasticity occurs when; i) environments are heterogeneous, ii) environmental signals are reliable, iii) benefits of inducing plasticity are larger than the costs, and iv) plasticity has a genetic basis (reviewed in Scheiner, 1993; Berrigan \& Scheiner, 2004; Ernande \& Dieckmann, 2004). Especially quantitative genetic and optimality models have been used to analyse the evolution of phenotypic plasticity. Where quantitative genetic models focus on the role of genetic constraints, optimality models focus on the ecological conditions (environments) affecting the evolution of phenotypic plasticity (reviewed in Berrigan \& Scheiner, 2004; Ernande \& Dieckmann, 2004). Although both methods have their strength and weaknesses, optimality models are especially useful to study selection for phenotypic plasticity while considering the effect of the environment, which is the focus in this thesis.

Optimality models have been used by evolutionary (plant) biologist to analyse trait selection in different environments (Parker \& Maynard Smith, 1990). This theory assumes that the individual, with its associated traits, that has the highest fitness in a given environment will be selected for. When individuals differ in traits and in fitness among environments, different environments will select for different individuals with their specific traits. As phenotypic plasticity can enable an individual to express different phenotypes in different environments, optimality models could analyse if and what kind of phenotypic plasticity will be selected for. In general, optimization theory can be divided into two categories; simple optimization and competitive optimization (Parker \& Maynard Smith, 
1990). The main differences between these two is that competitive optimization assumes that the surrounding individuals (with their traits) affect the fitness of a focal individual, where as in simple optimization only the trait of the focal individual determines its own fitness. When regarding competition for light, considering competitive optimization is required as the performance of one individual clearly depends on the traits of the others (Weiner, 1990). A common way to apply this competition optimization is performing an evolutionary game theoretical (EGT, see Glossary) analysis (Parker \& Maynard Smith, 1990; Falster \& Westoby, 2003; McNickle \& Dybzinski, 2013).

\section{Evolutionary game theoretical principles}

In general, EGT models take into account that the fitness of an individual depends on the presence of other individuals in the population and selection occurs if the fitness of an individual is higher than that of others. EGT models often reason from a perspective that a population consist of genetically similar individuals (also referred to as resident population) but that there can be an invader (also referred to as mutant) that has a different trait because it is genetically different. A mutant, which is present in low frequency in the population, could change the composition of the population if it has a higher fitness than individuals from the resident population. The way natural selection will be analysed depends on the assumptions made about how this mutant invasion affects the next generation. This can be done by choosing a given (replicator) rule. The simplest replicator rule is that of the winner takes all. This assumes that if the mutant individual with a different trait has a higher fitness than an individual of the resident population, the population will change into a monoculture population with the trait of the mutant (new resident population). The population with a given trait that cannot be invaded by a mutant with another trait is referred to as an evolutionary stable population and the trait itself is referred to as an evolutionary stable strategy (ESS, see Glossary; Parker \& Maynard Smith, 1990). In this case strategy is a synonym for trait (trait value). It is important to note that the ESS will often not optimize fitness at the population level, which is referred to as a tragedy of the commons (reviewed in Rankin et al., 2007; Anten \& Vermeulen, 2016).

EGT models have been used extensively to determine ESS-traits regarding light competition (Iwasa et al., 1984; Anten, 2002, 2005; Pronk et al., 2007; Dybzinski et al., 2011, 
2013; Farrior et al., 2013; van Loon et al., 2014). Some of these models considered trait plasticity (Dybzinski et al., 2011, 2013; Farrior et al., 2013). However, these models focussed mainly on the expressed traits, not on selection for the plastic response itself. To explicitly study if different environments will select for different levels of plasticity, plasticity has to be regarded as a quantitative trait that describes the physiological response that is responsible for the phenotypic changes, as mentioned before. Consequently, applying EGT principles could analyse to what extent natural selection would favour a given plastic response that is responsible for expressing the best phenotypes in accordance with the environment.

\section{Phenotypic plasticity from an environmentally dynamic perspective}

As outlined in the previous paragraphs, phenotypic plasticity is an interesting phenomenon that can be studied from the perspectives of ecology, physiology and evolution. Especially, to determine to what extent variation in plasticity affects competition and in turn to what extent the variation in plasticity results from selection in different environments, these three perspectives need to be combined. One challenge linking these different perspectives is the ability to scale up from organ-level responses to consequences on whole-plant and population-level. Another challenge is that the induced changes in phenotype (due to the plastic response) can influence the environment that elicit the plastic responses themselves, resulting in a feedback loop. In the case of SAS responses, the R:FR ratio that induces the plastic responses is created by the (vertical) structure of the plants within a vegetation (Ballaré et al., 1987; Chelle et al., 2007; de Wit et al., 2012; Crepy \& Casal, 2015). The decrease in R:FR will induce plastic response that change the phenotype (i.e. structure) of the plants. This change in turn will change the R:FR ratio in the vegetation, therefore creating a feedback loop between phenotype and environment. In addition, the plant phenotype determines resource capture, such as light for photosynthesis. Capturing light will influence growth and therefore the phenotype of the plants. Thus, in a developing vegetation stand, plants capture light to grow and perceive R:FR that both drive phenotypic changes, which in turn change light for photosynthesis and R:FR conditions. This dynamic feedback will go on and on, and finally determine plant performance within a given environment. Functional-structural plant (FSP) models are able to capture these types of 
feedback systems regarding the light environment (reviewed in Vos et al., 2010).

\section{Functional-structural plant model}

In general, FSP models simulate plant and vegetation structures in three dimensions (3D) interacting with the above- and belowground environment (reviewed in Vos et al., 2010; Dunbabin et al., 2013). FSP models have their origin in models of plant architecture, which started as purely mathematical descriptions, but over the years gained more biological functionality (Prusinkiewicz \& Lindenmayer, 1990; Prusinkiewicz \& Runions, 2012). In FSP models, depending on the research focus, detailed organ structures, physiological processes and interactions between plant architecture and environmental conditions are taken into account. Plant architecture consist of different organs that have a specific 3D structure and orientation in the environment and interact with an environmental factor. This interaction with the environmental factor is based on physiological processes such as photosynthesis and water-driven organ extension. A distinction can be made between descriptive and mechanistic FSP models. In descriptive models, plant growth is described according to fully empirical rules directly derived from experimental data (among others Da Silva et al., 2014; Zhu et al., 2015). In contrast, plant growth in mechanistic FSP models is based on the interaction with the environment and the physiological processes driving growth (Fournier \& Andrieu, 1998). Usually, even in mechanistic FSP models not all traits are simulated mechanistically; for instance leaf appearance rates and organ orientation angles may still be modelled empirically (Evers \& Bastiaans, 2016). In both types of FSP models however, structural traits and physiological processes at the organ level are scaled up to whole-plant phenotype and performance.

To explicitly simulate the light environment within a canopy and calculate the level of absorbed and perceived light per organ, FSP models can use a raytracing method (Chelle \& Andrieu, 1999; Hemmerling et al., 2008). In general, the raytracing method traces all light rays that travel from the light source into the virtual vegetation, up to every organ. When a light ray hits an object (e.g. a leaf), it can be absorbed, reflected or transmitted, depending on the optical properties of the object and on the waveband the ray represents. For example, $\mathrm{R}$ light will be mostly absorbed whereas $\mathrm{FR}$ light will be mostly reflected, leading to the R:FR ratio within the vegetation that can decrease based on the (vertical) structures of 


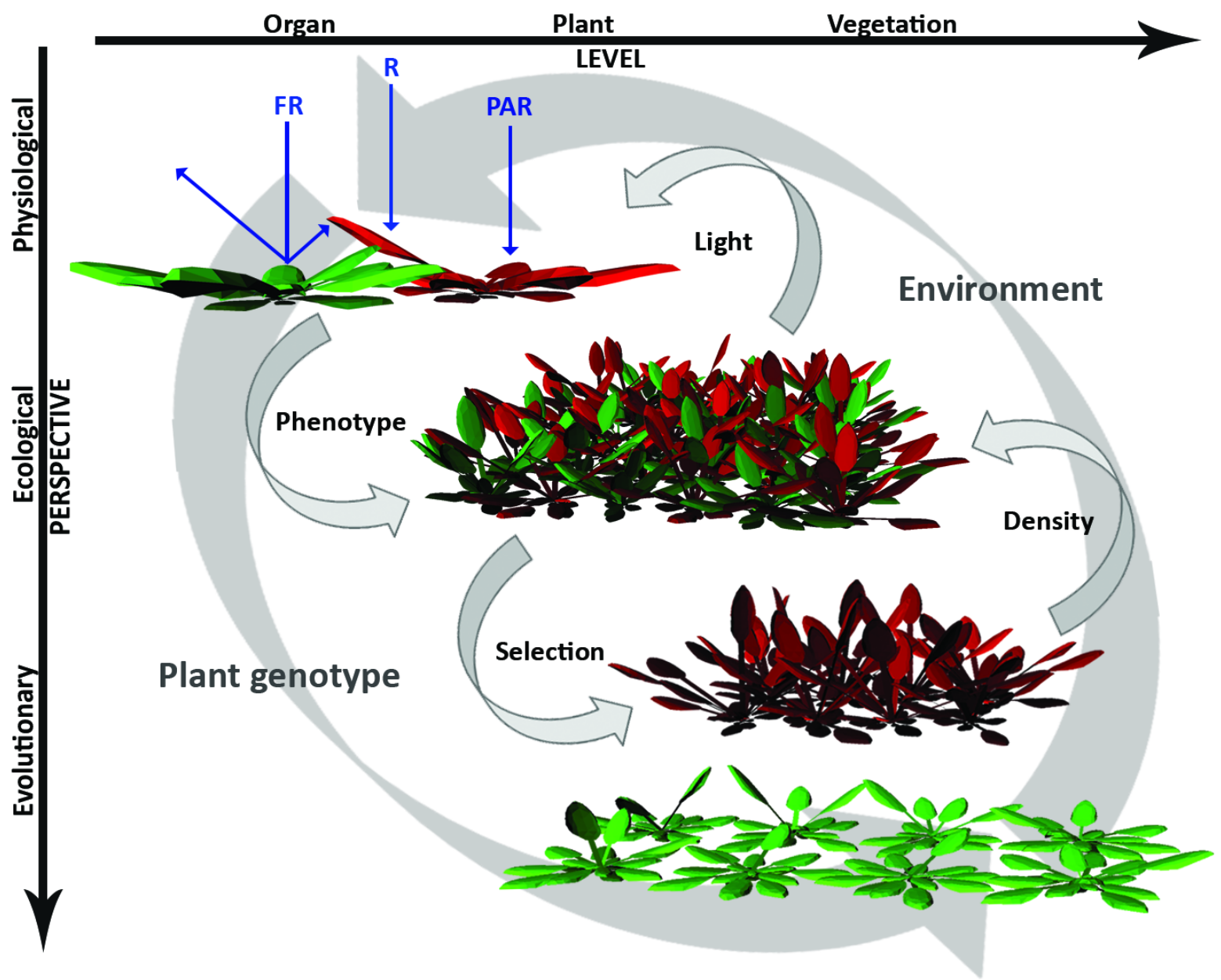

Figure 1.4 Visualisation on how a functional-structural plant (FSP) model scales up from organ level to plant and vegetation level and therefore links different plant research perspectives. Plants represent Arabidopsis plants consisting of petioles and laminas, and green and red coloured plants illustrate different genotypes. The FSP model simulates organ-level structures that interact with the light environment (representing photosynthetic active radiation (PAR), red ( $R$ ) and far-red (FR) light). The interaction between organ structures and the light environment represents physiological processes that drive phenotypic growth. The heterogeneous light environment is created by the developing phenotypic structures of the plants in the vegetation itself. Through the $3 \mathrm{D}$ orientation of the plant structures within the vegetation the degree of light competition is simulated explicitly, which determines final plant performance. Therefore, plant performance is assessed by the plant's own R:FR responses and that of the neighbour plants in the vegetation, and is the driving factor of selection. Depending on the population density, plants with different plastic response curves will be selected for (illustrated by the red genotype that is selected for in a higher density than the green genotype). Thus, the (physiological) responses at the organ level define how plants compete for light, and the competition processes at the vegetation level determine which responses are selected for in a given environment. Eventually the FSP model simulates light competition between plant genotypes (with specific plastic responses) that create their own (light) environment, and in turn, this environment determines the selection process for the plant genotypes. This way the FSP model can link physiological, ecological and evolutionary processes and scaled up from organ to plant and vegetation level. 
the plant organs. Light for growth is often described by a waveband that represents photosynthetic active radiation (PAR, $\lambda=400-700 \mathrm{~nm}$ ). PAR absorption will then drive photosynthesis and eventually determine biomass accumulation per individual plant. Studies have determined the role of structural traits during light competition (Barillot et al., 2014; Zhu et al., 2015; Evers, 2016) or simulated how R:FR light signals determine plant phenotype (Gautier et al., 2000; Evers et al., 2007; Kahlen \& Stützel, 2011). However, no study yet has combined plant growth based on light capture and phenotypic changes due to R:FR signalling at the same time.

\section{This thesis}

In this thesis, I analyse how variation in the level of phenotypic plasticity affects competition and how this variation could result from selection in various environments. As case study I focus on the petiole elongation and upward leaf movement (hyponasty) responses in the annual rosette plant Arabidopsis thaliana (Brassicaceae) in response to the proximity of neighbour plants, signalled through the R:FR ratio. Growing Arabidopsis in low versus high population density results in two distinct phenotypes: a flat rosette with short petioles in low density and increased leaf angels and increased petiole lengths in high density (Figure 1.1). Working with Arabidopsis allows me to use mutants that could express variation in plastic responses and identify players regarding the underlying physiological processes.

To link physiological, ecological and evolutionary perspective and scale from plastic responses at the organ level to whole-plant performance at the vegetation level, I use a FSP model that regards the dynamic feedback between light environment (PAR, $R$ and $F R$ ) and plant phenotype. Results of competition between plants at the vegetation level will define the selection processes, which determines the level of phenotypic plasticity that will be selected for, illustrating how evolutionary processes affect selection for a plastic responses that are underlined by physiological processes (Figure 1.4).

First, Chapter 2 outlines my view on why FSP models are an appropriate tool to scale from organ-level shade avoidance responses to plants in vegetation stands and thus assess implications of variation in these responses for plant and vegetation performance. I propose 
that understanding the ecological relevance of physiological processes and organ-level responses can be better understood through the feedback between plant experiments and model simulations.

In Chapters 3 and $4 \mathrm{I}$ focus on how variation in plastic responses determine light competition between plants by combining plant experiments and FSP modelling. In Chapter 3 the Arabidopsis FSP model is presented that scales from organ-level plasticity to whole plant performance while regarding the dynamic feedback between light environment and plant phenotype. The SAS responses included in the model were described by a plastic response curve and based on physiological plant experiments. By model simulations, and additional plant experiments for model validation, the effect of variation in plastic responses on plant competitiveness for light is analysed.

Chapter 4 presents the relationship between R:FR perception and induction of the two different phenotypic responses. Physiological experiments illustrate that different sites of R:FR perception are required to induce petiole elongation versus hyponasty. Simulations with the FSP model subsequently illustrate why these two plastic responses should be induced by their respective site of perception based on competition for light in various population densities.

In Chapter 5 I focus on how variation in plastic responses could be the result of selection at different environments by combining FSP modelling and evolutionary game theoretical (EGT) analysis. In this chapter, the Arabidopsis FSP model simulates light competition between plants with different plasticity levels, represented by different shapes of the plastic response curve. Based on the EGT principles I analyse if selection in different vegetation densities results in one plastic response curve that could dominate different vegetation densities.

In the general discussion (Chapter 6), I discuss the conclusions of this study by emphasizing on the role of the FSP model in combination with the plastic response curve to reach the main objective in this thesis. In addition, I discuss further ideas regarding analysing selection for phenotypic plasticity which depends on environmental conditions. 
CHAPTER 2

\title{
From shade avoidance responses to plant performance at vegetation level: using virtual plant modelling as a tool
}

\author{
Franca J Bongers ${ }^{1,2}$, Jochem B Evers ${ }^{1}$, Niels PR Anten ${ }^{1}$, Ronald Pierik ${ }^{2}$ \\ ${ }^{1}$ Centre for Crop Systems Analysis, Wageningen University, Wageningen, the Netherlands. \\ ${ }^{2}$ Plant Ecophysiology, Utrecht University, Utrecht, the Netherlands.
}




\section{Introduction}

The Shade Avoidance Syndrome (SAS) is one of the best-studied forms of plant phenotypic plasticity. The suite of SAS responses enables plants to accurately match their phenotype to the light conditions determined by neighbouring plants, especially the decrease in the ratio of red (R) and far-red (FR) light intensity (R:FR) (Ballaré et al., 1990).In recent years, significant progress has been made in understanding the physiological and molecular regulation of SAS (among others reviewed in Casal, 2013; Gommers et al., 2013; Pierik \& de Wit, 2014). In addition, several studies showed that SAS is adaptive because inappropriate elongation due to inaccurate estimation of neighbour proximity or deficiency in the capacity to respond to neighbour presence, is disadvantageous for fitness (Dudley \& Schmitt, 1995, 1996; Weinig, 2000; Pierik et al., 2003; Weijschede et al., 2008; Keuskamp et al., 2010). However, it is difficult to assess the consequences of detailed physiological and molecular regulations of SAS for whole-plant and whole-vegetation performance. In addition, the wide variety of different cues involved in plant-plant interactions, including light quality and quantity (Ballaré et al., 1990; Smith, 2000), mechanical interaction (i.e. touch and wind shielding; de Wit et al., 2012; Anten et al., 2005) and various volatiles (Pierik et al., 2003; Kegge et al., 2013) pose questions about their relative importance for plant performance. Here, we argue that the consequences of physiological regulations and the complexity of natural systems can be addressed by using virtual plant simulation modelling in combination with experimental studies. So called functional-structural plant (FSP) models have been applied in a broad range of research questions in the field of plant sciences (reviewed in Vos et al., 2010; Guo et al., 2011; DeJong et al., 2011; Prusinkiewicz \& Runions, 2012) and simulate plant development over time in three dimensions (principles are outlined in Box 2.1). These models can include responses to environmental conditions such as light (Figure 2.1) and can be used to study how the interplay between physiology, architecture and environment scale from plant organ to whole-plant performance. FSP models can generate and test hypotheses about the influence of different environmental components on plant growth and development by including one component at a time. By comparing model predictions with naturally developed vegetation stands, the contribution of the different components of environmental factors for plant performance can be assessed. 
In this letter we outline how FSP models can improve experimental designs, and that data collected from these experiments in turn can improve the mechanistic description of regulation of shade avoidance in the model. Ultimately, this feedback process results in a modelling tool that can scale up from plant organ responses to whole-plant performance and address ecologically relevant questions such as the adaptive significance of variation in SAS.

\section{Box 2.1 Creating a functional-structural plant (FSP) model}

An FSP model simulates development of plants and vegetation in three dimensions. In FSP models, plant architecture is defined by physiological processes that are affected by environmental conditions. Plant architecture is typically described at the (sub-)organ level (leaf, petiole, etc.). These components are organized in phytomers, the repeating basic building block of plant architecture. The connection, orientation and spatial location of the organs within a phytomer and between the phytomers results in a threedimensional architecture of the plant.

To create a dynamic model of plant development, data on organ size, position and orientation at different time points during development are required (Mundermann et al., 2005, Vos et al., 2010). Plant growth and development can be simulated descriptively (based on input data) or mechanistically (based on processes such as light absorption, photosynthesis and biomass growth) (e.g. Evers et al., 2010). To include photosynthesis, appropriate models such as a photosynthesis-light response functions can be implemented at the leaf level, or even parameters such as dark respiration, light-saturation, nitrogen content, water content, $\mathrm{CO}_{2}$ and $\mathrm{O}_{2}$ concentration and efficiency of the electron transport pathway can be implemented (Vos et al., 2010). To simulate light, a light source is virtually included, which can be a single or diffuse light source with light rays of different wavelengths. Eventually, to be able to create organ plasticity such as SAS responses, quantified dose-response curves of petiole length to R:FR ratios have to be determined and implemented at the organ level. To simulate light absorbance and reflection at the organ level, leaf optical properties (absorbance, reflectance and transmittance for red, far-red and photosynthetically active radiation (PAR)) are required as input. Finally, FSP modelling can be extended to include e.g. calculation of the leaf energy balance (e.g. Chelle, 2005) or the distribution pathogens (Everhart et al., 2011) factors that are known to interact with SAS. 


\section{Signal-response feedback}

Scaling from individual shade responses to the structure and performance of plants under natural conditions is complicated partly because it involves a complex feedback between cue and response.The architecture of a plant determines the light conditions in a canopy. These light conditions, e.g. R:FR, will in turn affect plant physiological processes that regulate plant architecture, which in turn modifies the light conditions and associated cues. The degree to which this interplay between perception of cues and associated responses are spatially integrated in plants is important for this process, but it is still poorly understood. For example, the extent to which signal detection and responses are spatially and temporally segregated remains debated (Casal \& Smith, 1988a,b; Ballaré et al., 1990; Kozuka et al., 2010). It is also unknown how plants integrate neighbour-derived signals with signals that are associated with other stressors such as defence against pathogens and herbivores (Izaguirre et al., 2006; de Wit et al., 2013). FSP models can quantify the strength and distribution of different light signals in plant canopies and consider different scenarios for spatial patterns of perception of these signals during the development of the vegetation (de Wit et al., 2012). This can help design experimental setups and treatment levels (e.g. R:FR) to reliably represent those under natural vegetation conditions, thereby improving the ecological relevance of laboratory and growth chamber experiments. Incorporating the understanding provided by results of better-designed experiments in FSP models can capture the feedback between plant architecture and environment, therefore in turn improving simulations of plant architecture in developing stands (Gautier et al., 2000; Evers et al., 2007). In addition, sensitivity analysis of FSP models can be used to test if the degree of signal detection and signal integration influence whole-plant performance in different environmental settings, linking mechanistic knowledge to ecological relevance. In summary, iterative cycles of experimentation and modelling have the potential to facilitate the analysis of the dynamics of complex vegetation development and combine mechanistic knowledge at different levels of organization, allowing a more holistic understanding of plant-plant interactions. 
(a)

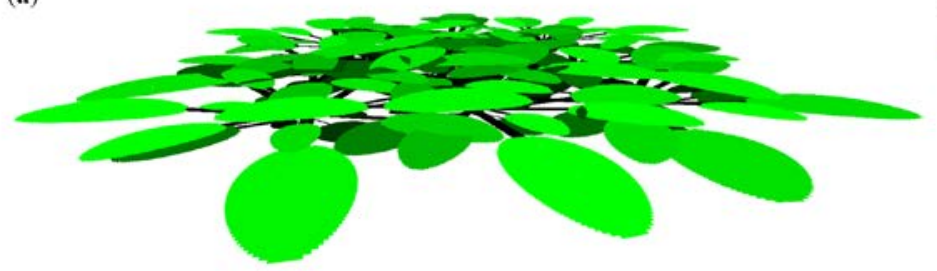

(b)

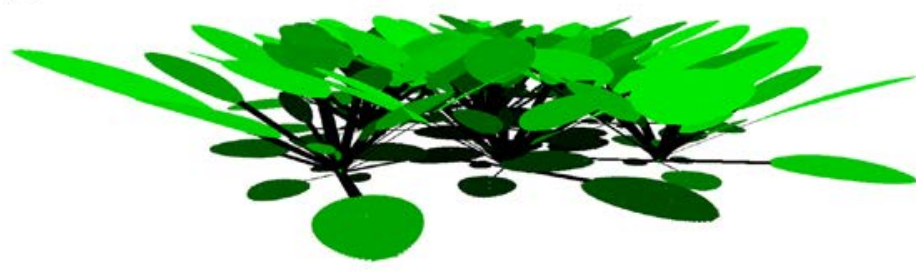

(c)

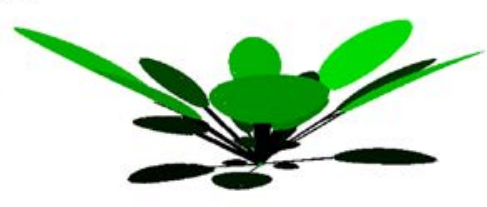

(d)

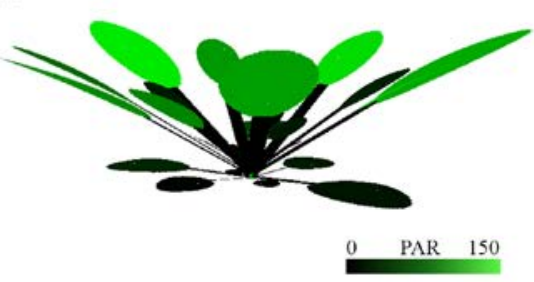

Figure 2.1 Simulated Arabidopsis plants after 35 days of growth using an FSP model (see Box 2.1 for a brief explanation on creating FSP models). Leaf Area Index is 2.8 in all cases. Shown are stands of $3 \times 3$ plants (a-b) and, for visual purposes, the middle plant isolated from a stand (c-d), resulted from different scenarios in which different SAS responses, increase petiole angle and increased petiole elongation, are simulated. (a) illustrates no response to neighbour plants. (b) illustrates only increased petiole angle in response to neighbour plants. (c) illustrates an isolated plant from a stand in which plants only increased their petiole angle in response to neighbour plants. (d) illustrates both increased petiole angle and increased petiole elongation in response to neighbour plants. Plant architecture was based on data from de Wit et al., 2012, Mundermann et al., 2005 and experiments conducted by FJB (19 mL pots, 9 hours light at $200 \mu \mathrm{mol} \mathrm{m} \mathrm{m}^{-2}$ PAR and R:FR of $1.8,15$ hours dark, $20{ }^{\circ} \mathrm{C}$ and $70 \% \mathrm{RH}$ ). In this model leaf area development was independent of absorbed light and leaf angles were simulated in accordance with experimentally observed changes over time. No feedback relations on various physiological parameters, such as photosynthesis characteristics, leaf anatomy, nutrients and more are incorporated in this highly simplified model example. Petiole length was calculated from empirical petiole growth data of isolated plants and multiplied by a factor derived from a dose-response curve relating increased elongation to perceived R:FR ratio. Light is simulated using the ray-tracing method (Chelle \& Andrieu, 1999), in which the fate of each light ray from a number of light sources representing diffuse light (Evers et al., 2007) is determined. Colour brightness of the leaves corresponds to the PAR absorbed by the leaves, ranging from 0 to $150 \mu \mathrm{mol} \mathrm{m} \mathrm{s}^{-1}$.

\section{Different SAS components}

SAS, being a syndrome, contains multiple coordinated structural and functional responses to changes in the light environment, e.g. upward leaf movement and increased petiole elongation in Arabidopsis thaliana (Pierik \& de Wit, 2014). It has been shown that plants that are impaired in SAS responses have a fitness disadvantage when competing for light with plants that show wild type shade avoidance responses (Schmitt et al., 1995; Pierik et al., 2003; Keuskamp et al., 2010). However, the relative contributions and roles of different components of SAS in determining competitive power remain poorly understood. Genotypes 
used in these studies are typically impaired in multiple SAS components simultaneously. Using FSP models, plants can be simulated that differ in specific SAS components, which can help to identify the impact of the specific responses on competition in vegetation stands (Figure 2.1 and 2.2), as well as their combinations. As a highly simplified example, Figure 2.2a illustrates how increased petiole angle slightly reduces total absorbed photosynthetic active radiation (PAR) by the whole rosette, whereas increased petiole elongation in response to decreasing R:FR ratio does not have a substantial effect. The slightly reduces PAR absorption at higher leaf angle is probably the result of inefficient capture of light photons due to vertical leaf position (e.g. reviewed in van Zanten et al., 2010), rather than perpendicular to the directions of the light. In this simulation all plants in the vegetation stand respond similarly to the environmental signals they perceived. When assuming that there is variation between plants and surrounding plants have different trait values, an increased petiole angle will crucially determine individual plant PAR absorption (Figure 2.2b). Having a slightly increased petiole angle compared to the surrounding plants has a considerable benefit in terms of light absorption. This probably results from this plant overtopping its neighbour plants, thereby preventing it from being shaded and this benefit is apparently stronger than the slightly reduced photon capture of elevated leaves. Here, we show that FSP model simulations can help to better understand the effects of (variation in) different SAS components on whole-plant performance, and in response to the environment. This will provide information useful for the design of later ecophysiological experiments.

\section{Adaptive significance of natural variation in SAS}

Ecological theoretical studies indicate that there is strong natural selection for finely tuned SAS responses that enable plants to optimally adjust to the complex temporal and spatial dynamics of their surrounding vegetation (see reviews by Falster \& Westoby 2003; Anten \& During 2011). However, the question as to what would be an optimal combination and intensity of SAS responses under different environmental conditions has been seldom assessed quantitatively, and thus little is known about how natural selection may have acted on variation in SAS responses. We propose that the combination of FSP modelling and evolutionary game theory (EGT) can help address this problem. EGT is a mathematical tool to 

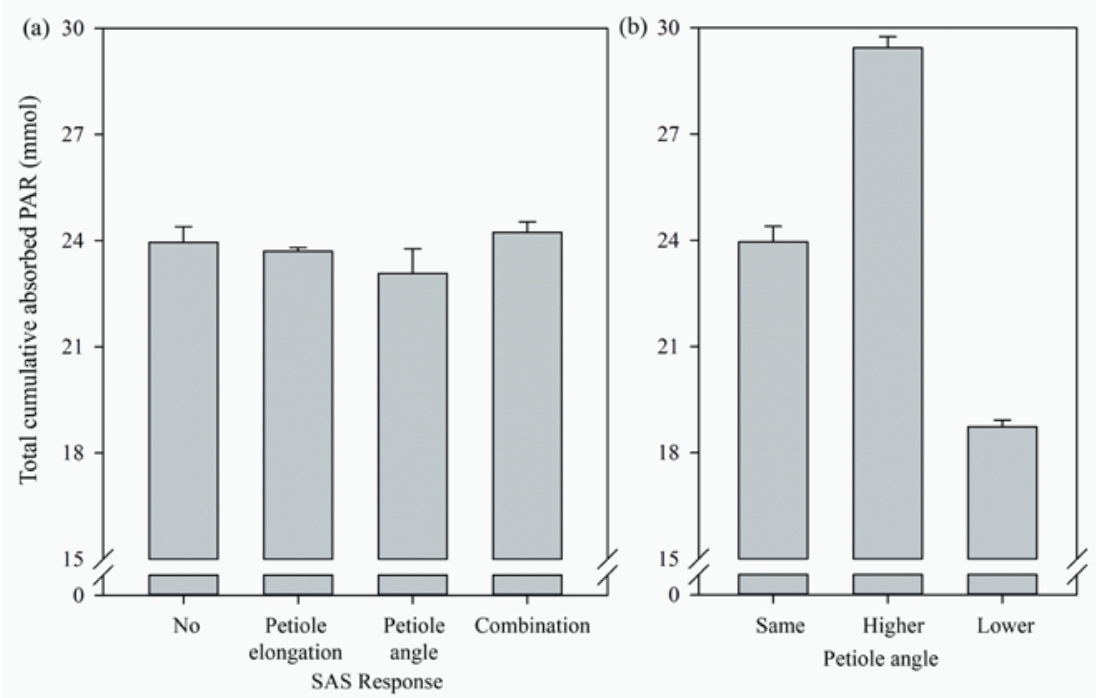

Figure 2.2 The impact of variation in shade avoidance properties on light interception: illustrating the mechanics of a simplified model. Total cumulative absorbed photosynthetic active radiation (PAR) by the plant after 35 days (total plant leaf area $11.8 \mathrm{~cm}^{2}$ ) was calculated. Different scenarios were simulated in which all plants in the stand had the same physiological characteristics (a) or in which the middle plant was different from the surrounding plants (b). In (a) 'No' indicates no response to neighbour plants resulting in a flat stand (corresponding to Figure 2.1a).'Petiole elongation' indicates only increased petiole elongation in response to perceived R:FR ratios. 'Petiole angle' indicates only increased petiole angle in response to neighbour plants (see Fig. 1b,c). 'Combination' indicates both increased petiole elongation and petiole angle in response to neighbour plants and perceived R:FR ratios (see Figure 2.1d). In (b) 'Same' petiole angle indicates that the focal plant had the same petiole angle as its neighbours. 'Higher' and 'Lower' indicates that the petiole angle of the focal plant was slightly more or less inclined than its neighbours, respectively. The simulated vegetation stands consisted of a stand of $3 \times 3$ plants (interplant distance was $2 \mathrm{~cm}$ ), the middle plant (focal plant) was used for analysis, and the average of six simulations was reported (error bars show SE). The model was run at hourly time steps, included day and night periods (9 hours light, 15 hours dark), and simulated light at an intensity of $200 \mu \mathrm{mol} \mathrm{m} \mathrm{m}^{-2}$ PAR and an R:FR ratio of 1.8. Further model details are described in the caption of Figure 2.1

analyse natural selection on traits in situations where organisms interact with each other (Iwasa et al., 1984; Parker \& Maynard Smith, 1990; Falster \& Westoby, 2003; Anten \& During, 2011). EGT considers that the payoff of a given strategy for a plant depends on the characteristics of its neighbours, and can derive so-called evolutionary stable strategies (ESS) whereby a potential invader with a different trait value cannot invade the population. Going back to the example depicted in Figure $2.2 \mathrm{~b}$ where plants with slightly inclined leaves where favoured in light competition. Such plants could thus invade creating a new population with more inclined and as shown in Figure 2.2a less light capture. Using FSP modelling this procedure can repeated until an ESS is reached at which no unilateral change in leaf angle can give a plant competitive example. Thus a combination of FSP and EGT can be used to simulate how natural selection acts on variation in SAS and how this in turn affects vegetation functioning. Summarizing, FSP modelling, experimental research and EGT could 
be combined to assess how physiological and molecular mechanisms of shade avoidance might affect plant performance and be targets for natural selection to drive the development of stable vegetation stands.

\section{Conclusion}

SAS is one of the best-studied examples of phenotypic plasticity and impressive progress has been made in understanding its physiological regulation. In addition, several studies showed that SAS is beneficial in competitive environments. We propose that the link between physiological regulation of shade avoidance at the organ level and whole-plant performance in vegetation settings can be better understood by iterative cycles of FSP modelling and experimentations. Eventually, such models can be used to analyse the effect of natural variation in SAS on the performance of different plant phenotypes in natural vegetation stands.

\section{Acknowledgements}

Funding is provided by the Netherlands Organisation for Scientific Research (ALW grant $\mathrm{nr}$ 821.01.014 to NPRA, VIDI grant nr 864.12.003 to RP). 
CHAPTER 3

\title{
Subtle variation in shade avoidance responses have profound consequences for plant competitiveness
}

\author{
Franca J Bongers ${ }^{1,2}$, Ronald Pierik ${ }^{2}$, Niels PR Anten ${ }^{1}$, Jochem B Evers ${ }^{1}$ \\ ${ }^{1}$ Centre for Crop Systems Analysis, Wageningen University, Wageningen, the Netherlands. \\ ${ }^{2}$ Plant Ecophysiology, Utrecht University, Utrecht, the Netherlands.
}




\section{Abstract}

Although phenotypic plasticity has been argued to be beneficial for plant performance, there is limited knowledge on how variation in plastic responses has consequences for plant performance in competition for light. We used a novel combination of detailed plant experiments and functional-structural plant modelling that captures the complex dynamic feedback between the changing plant phenotype and the within-canopy light environment in time and 3D space. As plastic trait we regarded plastic responses to an environmental signal. As a case study for plant plasticity, we focussed on leaf angle increase and petiole elongation rates to changes in light quality, which are two shade avoidance responses in Arabidopsis. We measured and then implemented these responses into a 3D functional structural model, by which plant phenotype was an emergent property of the model simulations. Our results showed that substantial differences in competitiveness may arise between genotypes with only marginally different hyponasty or petiole elongation response levels, due to the amplification of plant growth differences by small changes in plant phenotype. These results indicate that selection pressure is likely to have played a role in fine-tuning the sensitive shade avoidance responses found in plants. As our modelling approach simulates the dynamic interaction between phenotype and environmental conditions and regards variation in plastic responses allows to step towards analysing natural selection for plasticity. 


\section{Introduction}

Plants compete for resources with their neighbours, which influences species composition and vegetation dynamics in both natural (Kiaer et al., 2013; Kunstler et al., 2016) and managed plant communities (Olsen et al., 2005; Yu et al., 2015). Plants experience both above and belowground competition, and the relative importance of the degree of competition for plant performance depends on the availability of resources, e.g nutrient level or light availability (Kiaer et al., 2013). The degree of competition for resources and therefore of plant functioning and performance is influenced by differences in plant phenotype, created by its component traits and the values of those traits (Kunstler et al., 2016). These trait values can be genotype specific but may also be modulated by environmental factors through phenotypic plasticity. Phenotypic plasticity has commonly been defined as the ability of a genotype to express multiple phenotypes in various environments (see e.g. Bradshaw 1965; Sultan 2000; DeWitt \& Scheiner 2004). Here we emphasize that expression of different phenotypes in different environments is mediated by dynamic organ-level responses to signals evoked by the environment. From an evolutionary perspective one can argue that plants have evolved to optimize plastic responses to maximize resource acquisition in different environments (Sultan, 2000). Plastic responses to changes in vegetation density and the associated light conditions constitute a well-known form of phenotypic plasticity in plants, called the shade avoidance syndrome (SAS; reviewed in Franklin, 2008; Casal, 2012; Gommers et al., 2013; Ballaré \& Pierik, 2017). An increase in stem or petiole extension rate, a reduction in branch production, an increase in leaf inclination (hyponasty) and an advanced flowering time are typical SAS responses that plants exhibit when encountering increased competition for light, though the combination of responses differ between species.

Relations between species, component traits and their values, and their relationship with competitiveness have been studied intensively and used to understand ecosystem processes (Dybzinski et al., 2011; Farrior et al., 2013; Bardgett et al., 2014; Kunstler et al., 2016). For instance, game theoretical studies suggest that because plants compete for resources, plants can evolve traits that are related to relative more investment in resource harvesting (e.g. leaves, stems and roots) instead of reproduction. This means that under 
competition, natural selection can result in plant traits that will not optimize performance of the whole-plant population, also referred to as tragedy of the commons (reviewed in Falster \& Westoby, 2003; Rankin et al., 2007; McNickle \& Dybzinski, 2013). The existence of a tragedy of the commons may have great consequences for vegetation performance and functioning (Anten \& Vermeulen, 2016). However, studies that evaluate the role of resourceharvesting traits for competition often not take phenotypic plasticity into account. Analysing how plastic responses affect competition is challenging because plastic responses affect trait values that influence the dynamic interaction between plant phenotype and environmental conditions and signals. Environmental signals elicit plastic responses that induce small trait changes which in turn change the light climate and thus modify the environmental signals. Furthermore small changes early in plant development eventually can be amplified into substantial consequences for competitiveness. Although phenotypic plasticity can be beneficial for plant performance, illustrated by adequate stem or petiole length matching to different environments (Schmitt et al., 1995; Dudley \& Schmitt, 1996; Pierik et al., 2003; Weijschedé et al., 2008), it is unknown to what extent variation in the plastic response itself has consequences for plant performance in competitive settings.

Our main objective is to determine to what extent differences in trait plasticity between neighbouring plants affect the outcome of competition for light, considering the dynamic feedback between plant phenotype and environment. We use SAS responses in Arabidopsis (Arabidopsis thaliana) as a case study for phenotypic plasticity. Arabidopsis rosettes show two major SAS responses: increased leaf angle (hyponasty) and petiole elongation (reviewed in Pierik \& de Wit, 2014). When Arabidopsis plants are grown in dense population stands, leaf angles will first increase due to physical touching among growing leaves (de Wit et al., 2012). This resulting vertical stand structure will change the ratio of red to far-red (R:FR) light scattered by the elevated leaves. This decrease of R:FR light is the most important signal for the subsequent induction of leaf hyponasty and petiole elongation (Pierik \& de Wit, 2014). To quantify the effect of differences in shade avoidance responses on plant performance in competitive settings, we used a novel combination of detailed plant experiments and functional-structural plant (FSP) modelling (outlined in Figure 3.1). FSP models can capture the complex dynamic feedback between the changing plant phenotype and the surrounding light environment by simulating plant phenotypic development and 
biomass growth over time in three dimensions at the organ level (Vos et al., 2010; Evers, 2016). In this model, the ability of plants to exhibit plastic responses is regarded by using response curves; dose-response relationships or step-wise relationships that relate organ trait change to a range of R:FR conditions (Gautier et al., 2000; Evers et al., 2007; Evers \& Vos, 2013). By simulating the R:FR distribution as a function of the dynamic 3D plant phenotypes that are created by the interaction of resource acquisition and growth at the organ level, the plastic responses at the organ level are quantitatively linked to whole-plant performance during competition (Chapter 2; Bongers et al., 2014).

\section{Material and Methods}

\section{Plant experiments}

Three independent experiments were conducted to obtain organ level growth data, petiole elongation response curves, and plant phenotype and performance of Arabidopsis genotypes, for model design and validation (outlined in Figure 3.1). To obtain organ level growth data, wild-type Col-0 plants were used, for the other two experiments also the genotypes rot3-1 (Kim et al., 1998) and hfr1-5 (Sessa et al., 2005) were used because of their distinct levels of petiole elongation (illustrated in Figure 3.2). Arabidopsis seeds were sown on potting soil (mix Z2254, Primasta B.V., the Netherlands), stratified for 4 days at $4^{\circ} \mathrm{C}$ in the dark after which they germinated and grew in a growth chamber with 9-hour photoperiod of $200 \mu \mathrm{mol} \mathrm{m} \mathrm{m}^{-2}$ PAR and R:FR ratio of $2.3,20{ }^{\circ} \mathrm{C}$ and $70 \%$ relative humidity. Ten days after germination, seedlings were transplanted to individual $19 \mathrm{ml}$ pots $(\varnothing 2.5 \mathrm{~cm})$ and plants grew in the same growth chamber with bottom up watering for soil water saturation.

\section{Experiments for model design}

To obtain organ-level growth data, Arabidopsis wild-type Col-0 was grown solitarily (referred to as 'low density' in the results) or in high density stands of $7 \times 7$ plants with inter plant distance (IPD) of $2.5 \mathrm{~cm}$, until bolting. Between day 21 and day 46, plants were harvested every 2-4 days, and per harvest two high density stands and 10 individually grown plants were selected. Per stand the outer two rows of plants were excluded from the harvest to diminish possible border-effect. Before every harvest, leaf angle of leaf rank number 8 and 10 were measured with a protractor. Per harvested plant, laminas and petioles were 
scanned (at $600 \mathrm{dpi}$ ), while the order of rank was preserved. For all leaves with a rank higher than 6 and with a distinct petiole, all laminas were pooled separately from the petioles and dried in the stove for a minimum of 48 hours for respectively specific lamina and petiole dry weight. The remaining aboveground plant material was pooled and dried in the stove to finally get total aboveground biomass. Root material was not harvested. Leaf scans were analysed with open-source software ImageJ to collect petiole length and width and lamina area, length, width and shape. Petiole length and lamina area were used to determine parameter values for the organ growth function (see below Organ-level substrate allocation). Data of all harvested plants per developmental stage and density were used to calculate trait value averages. In Supporting Information Table S3.1 an overview is listed of all parameter values used in the model and extracted from this experiment.

To obtain specific petiole elongation response curves for three Arabidopsis genotypes, 10-day-old seedlings were transplanted in $70 \mathrm{ml}$ pots $(\varnothing 5 \mathrm{~cm})$ and grown until 28-day-old plants were subjected to one of eight R:FR ratio $(2.3,1.6,1.2,1.0,0.7,0.5,0.2$ and 0.1 ) for 24 hours. There were 12 replicated plants per R:FR level. These eight different $R: F R$ ratios were created by supplementing the normal light ( $R: F R ~ 2.3)$ with FR LEDs (730 nm; Philips Green Power, The Netherlands). Per plant, two petioles (start length 4 - $6 \mathrm{~mm}$ ) were measured at the start and end of the experiment with a digital calliper ( 3 decimals). Per petiole the relative elongation was calculated and the mean of the two petioles per plant was used for further analysis. The relative elongation values of all genotypes at every R:FR value were described with the following function:

$$
\mathrm{P}=\mathrm{b} * \mathrm{R}: \mathrm{FR}^{-a}
$$

where $\mathrm{P}$ is the relative petiole elongation $\left(\mathrm{mm} \mathrm{mm}^{-1} 24 \mathrm{~h}^{-1}\right)$, $a$ determines the slope of the function and $b$ is the elongation rate at a R:FR of 1 . Per genotype separately, the specific parameter values for this function were fitted.

\section{Experiments for model validation}

Three different Arabidopsis genotypes were grown solitarily (low density) or in high density stands of $8 \times 8$ plants (IPD of $2.5 \mathrm{~cm}$ ) composed of plants of the same genotype (monoculture) or plants of two genotypes grown in a checker board pattern (mixtures) 


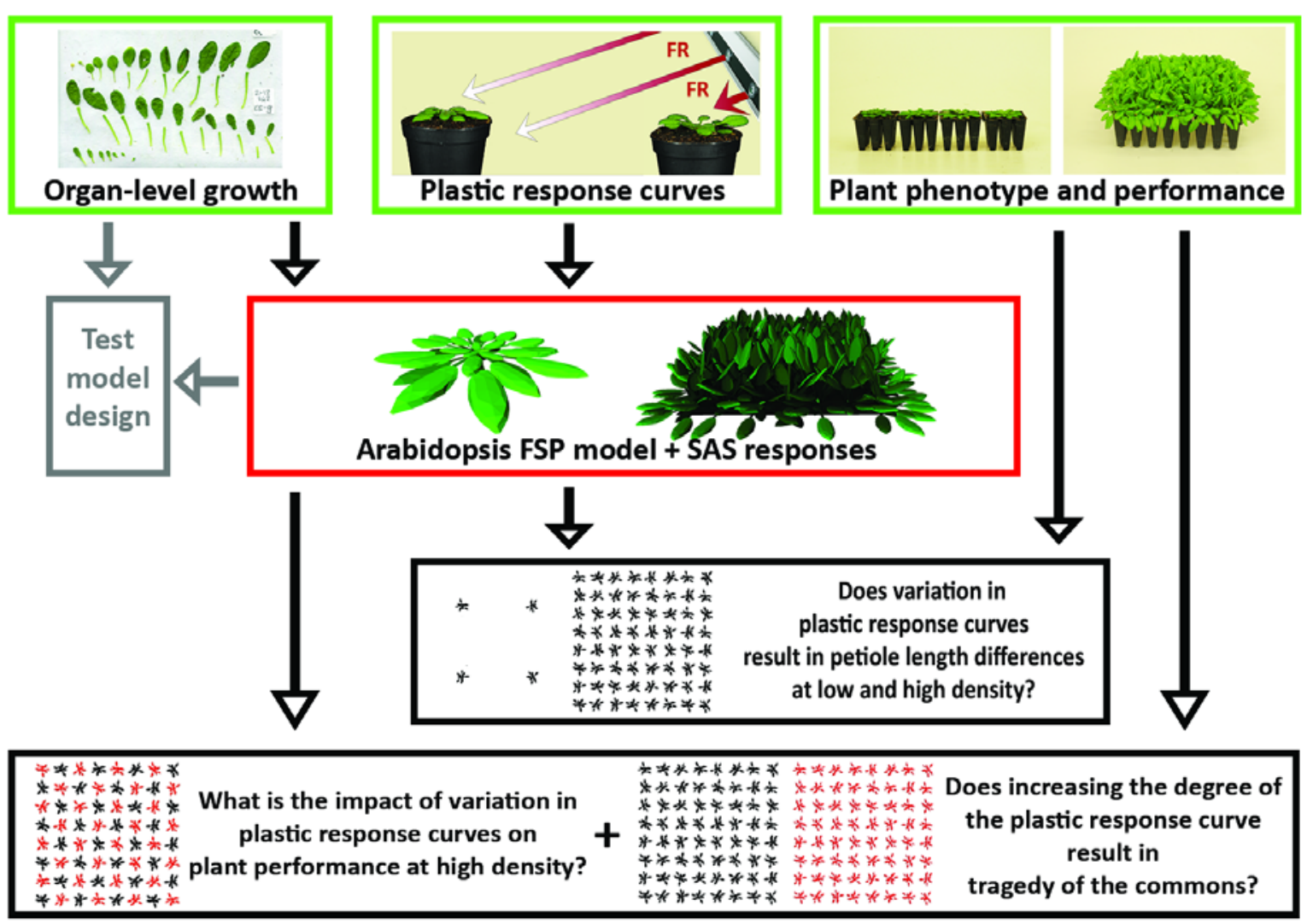

Figure 3.1 Overview of the experimental design, in which three independent experiments (bordered in green) are combined with functional-structural plant (FSP) modelling (bordered in red) to address three questions (bordered in black). Data of organ growth and detailed plastic responses of Arabidopsis were used to develop an FSP model that included two plastic responses of the shade avoidance syndrome (SAS); hyponasty and petiole elongation. The FSP model simulated plant growth in time and space, capturing the dynamic feedback of changes in plant phenotype and the surrounding light environment. The model design was tested by comparing data plant phenotypic and performance data from a plant experiment and model simulation (Scenario 1; bordered in grey). To answer three question, the model simulated plants with different SAS response values in low and high density (Scenario 2) or in monocultures and mixtures at high density (Scenarios 3-5). In addition, an independent plant experiment was conducted to obtain plant phenotype and performance data for three plant types with distinct petiole elongation response curves to validate model output. Detailed information about the experimental setup, and model description and scenarios is outlined in Material and Methods.

(Keuskamp et al., 2010). In total five replicated plots per genotype and stand and five individual plants per genotype were grown and harvested 46 days after germination. For all solitary plants and three plants per genotype per plot, the laminas and petioles were scanned (at $600 \mathrm{dpi}$ ), while the order of rank was preserved, and petiole lengths were analysed with ImageJ. For the remaining plants, only total lamina area per plant was scanned. All laminas were pooled separately from the petioles and dried in the stove for a minimum of 48 hours for respectively total lamina and petiole dry weight. Per plot the outer two rows of plants were excluded from the harvest to diminish a possible border effect. The 
mean value of the middle 16 or 8 individuals per genotype per plot respectively was calculated and used as independent value for further analysis. Paired student's T-test was used to test significant difference between genotypes within the mixture, and unpaired student's T-test was used to test significant difference between monocultures.

\section{Model description}

A functional-structural plant (FSP) model (Vos et al., 2010; Evers, 2016) of Arabidopsis rosette growth and development was constructed using the simulation platform GrolMP v1.5 (Hemmerling et al., 2008). In our FSP model, the rosettes were represented as a collection of leaves that were composed of petioles and laminas. The leaves individually grew in time in three dimensions based on light interception, photosynthesis and plant-wide carbon allocation principles. Therefore, simulated plant growth depended on the level of competition for light individual plants experienced with neighbouring plants: plant size and biomass were thus an emergent property of the model. To construct this plant model, parameter values for organ structure, physiological processes and environment signals have been obtained from dedicated experiments (described above, outlined in Figure 3.1) and from literature (see Supporting Information Table S3.1 for all parameters values). The complete model is available upon request from the corresponding author.

\section{Plant and organ structure}

All leaves that make up the rosette were constructed of an individually represented petiole and lamina. An additional root compartment functioned only as an invisible sink for assimilates, simulated by allocating a fixed ratio of total available substrates to the roots (i.e., nutrient and water uptake was not included in the model). Leaf initiation rate was described empirically using a plastochron value and a phyllotactic pattern. The leaves were provided with values for reflectance, transmittance and absorbance of PAR, R and FR light, which were used by the radiation model to simulate the light environment and calculate the absorption of PAR and perception or R:FR by the leaves. The petiole was represented by a flat rectangle with a fixed length : width $(\mathrm{L}: \mathrm{W})$ ratio until a maximal petiole width was reached and the petiole length increased without increase in width. The lamina was described by an oval shape with a ratio between the maximum width and the length, and curved edges representing realistic Arabidopsis leaf shape. The L:W ratio values did not 
change in time or in response to the environment. L:W ratio for the lamina was specified per rank number, to match observed variation. Each leaf was provided with an inclination angle sensitive to the R:FR ratio of the intercepted light, further specified in Shade avoidance responses below.

\section{Plant photosynthesis and substrates}

Plant growth was based on substrates provided by photosynthesis by every leaf organ independently, through PAR absorbed by the organ itself. Organ photosynthesis rate was calculated with a rectangular hyperbolic light response curve (Goudriaan \& van Laar, 1994) assuming a fixed photosynthetic capacity identical for all organ ranks and age. The photosynthesis rate per unit organ area per second was converted into daily $\mathrm{CO}_{2}$ assimilated per organ per day to match the model time step of one day (Evers \& Bastiaans, 2016). A daily time step is justifiable for climate chambers since the amount angular distribution of incoming radiation on the plants is constant during the day. The sum of assimilates produced by all organs resulted in whole-plant $\mathrm{CO}_{2}$ assimilation per day and these were converted into growth substrates per day by using $\mathrm{CO}_{2}$ molar mass and carbon biomass fraction values (Evers et al., 2010). Finally, deducting maintenance cost and adding any reserves from the previous time step (see Organ-level substrate allocation) resulted in the total amount of substrates for growth available each day (Evers \& Bastiaans, 2016).

\section{Organ-level substrate allocation}

The partitioning of substrates to growing organs was based on the relative sink strength principle (Heuvelink, 1996), assuming a central pool of substrates and consequently nonhierarchical assimilate allocation to sinks. The relative sink strength of an organ was expressed as a fraction of total plant sink strength, and determined the demand for substrates for each organ in relation to its age. When the total available substrates exceeded total plant sink strength, the remaining available substrates were saved as reserves and used in the next time-step (see above Plant photosynthesis and substrates). Organ sink strength was defined as its potential growth rate, and based on the beta growth function (Yin et al., 2003), a bell-shaped function of organ age which is the first derivative of a sigmoid function with three parameters representing the potential size, the growth duration, and the organ age at which the growth rate is maximal. These parameters were estimated independently 
for lamina and petiole, based on experimentally obtained organ-level growth data of Arabidopsis grown in low and high density (see Experiments for model design). Potential size was set to the maximal observed organ size, for lamina in area $\left(\mathrm{cm}^{2}\right)$ and petiole in length $(\mathrm{cm})$. Parameter values for growth duration and age at maximum growth rate were deducted from fitting the beta function to the obtained organ sizes that were normalized for organ age and maximal size. Only leaf rank numbers 7 up to 25 were used for analysis because for these leaves we had complete growth curves. During model simulations, the potential growth rate in size was converted into potential growth substrates by using values for specific leaf area (SLA) and specific petiole length (SPL), for respectively lamina and petiole. These SLA and SPL values were also used to convert the simulated accumulated organ biomass into organ dimensions. SLA was constant with size and age, and SPL decreased with petiole length.

\section{Shade avoidance responses}

Two shade avoidance responses of Arabidopsis were included in the model: hyponasty (by touching and by R:FR) and petiole elongation (by R:FR). Hyponasty by leaf touching was induced upon mechanical interaction at the tips of two growing leaves before the R:FR in a canopy decreases significantly (de Wit et al., 2012). Touch-induced hyponasty was simulated to occur when the distance between lamina tips of neighbouring leaves was smaller than 2 $\mathrm{mm}$. Hyponasty induced by R:FR perception was simulated to happen when the perception of R:FR by the lamina was below a threshold value of 0.5 . Every model time-step ( 24 hours) when these leaf touch or low R:FR thresholds were crossed the leaf's angle increased by a fixed number, for which either a default value of 16 degrees (based on measurements on Col-0) was used or another value depending on the model scenario (see below Model scenarios). The leaf angle over time was therefore a function of the number of time steps in which touch or low R:FR perception occurred, with a maximum leaf angle of 80 degrees. Leaves with rank number up to six could not become hyponastic.

The second SAS response incorporated in the model was relative petiole elongation related to the R:FR perceived. As default setting the petiole response curve based on Arabidopsis type Col-0 was used and was responding to R:FR ratio's perceived at leaf lamina level (Kozuka et al., 2010), for other settings see Model scenarios below. The fitted function 

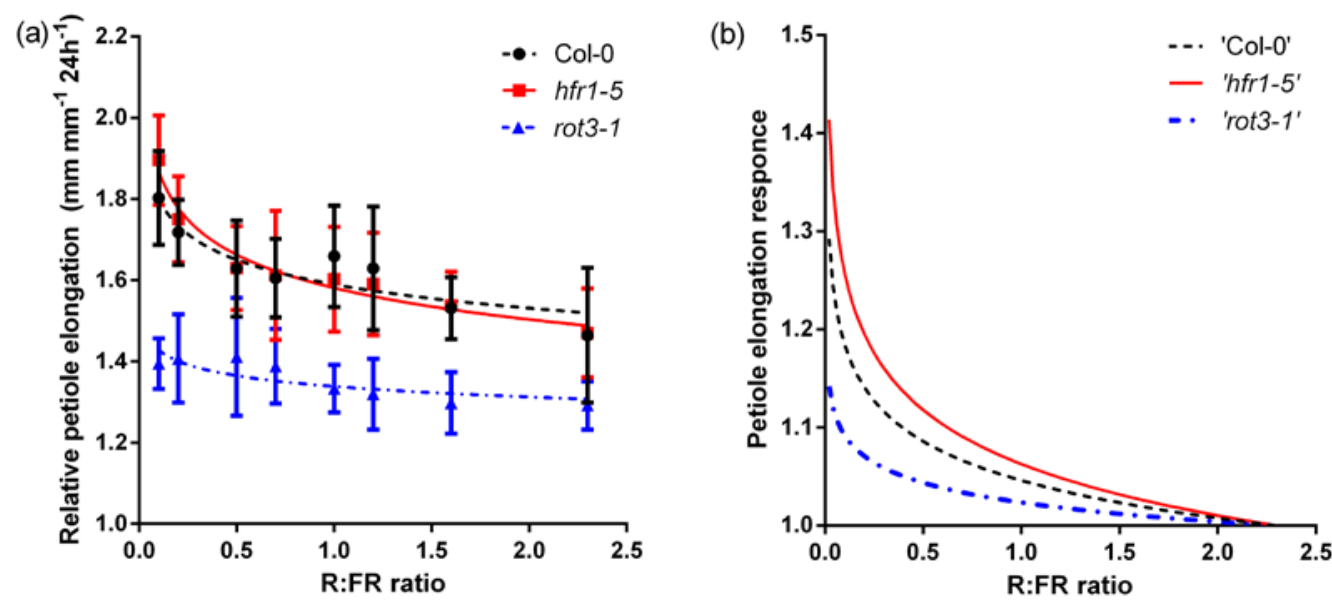

Figure 3.2 Petiole elongation response curves from three Arabidopsis genotypes. (a) Measured relative petiole elongation at different R:FR ratios for Col-0 (black - dotted), hfr1-5 (red-solid) and rot3-1 (bluedotted) with genotype specific fitted curves $\left(P=b * R: F R^{-a}\right)$. Experimental data represents mean $\pm S D(n=12)$. (b) Petiole elongation response curves for the corresponding Arabidopsis genotypes as used in the model, obtained by normalizing the fitted petiole elongation response curves for an R:FR of 2.3.

for the relative petiole elongation obtained from the petiole elongation experiment was normalized for growth at control R:FR light (R:FR 2.3). This way the relative petiole elongation rate could be simulated in addition to petiole growth by carbon allocation. Petiole elongation and related extra investment of substrates was modelled in two steps. First the petiole could elongate by multiplying the petiole length with the relative petiole elongation curve (representing cell expansion without extra biomass demand (Sasidharan et al., 2010; Huber et al., 2014)). Second, the longer elongated petiole increased its sink strength with the amount of substrates that were missing to fit the SPL value corresponding to length (representing increased biomass allocation to the petiole (Poorter et al., 2012; de Wit et al., 2015)). Petioles could only show the elongation response during the actual growth-phase of the beta growth function, i.e. when the petiole growth duration had passed, R:FR-induced elongation of that petiole did not occur anymore. Petiole length over time was therefore a result of daily calculated carbon allocation and petiole elongation based on R:FR perception.

\section{Model scenarios}

In all scenarios, plants were simulated solitarily (representing low density) or in dense monocultures or mixtures (consisting of $8 \times 8$ plants and IPD of $2.5 \mathrm{~cm}$ ) for 46 days, and 
different plant types were created by adjusting relevant SAS response values. In Scenario 1, three plant types were simulated solitarily and in monocultures to test the extent to which the model could simulate Arabidopsis phenotype and growth: two plant types had either no hyponastic responses ('noHypo') or no petiole elongation response ('noPE'). The third plant type had default SAS response values based on Arabidopsis wild-type (referred to as 'Col-0'). Dynamic changes of leaf angle, petiole length and total aboveground biomass of these plant types were compared with data from experimentally grown Col-0 Arabidopsis grown in low or high density stands (see Experiments for model design). In Scenario 2, to validate if variation in the petiole elongation response curve could result in petiole length differences at low and high density, we simulated two plant types with different values for their petiole elongation curves ( 0.073 for ' $h f r 1-5$ ' and 0.028 for 'rot3-1' plant type, based on the $h f r 1-5$ and rot3-1 Arabidopsis genotypes) in low and high density stands. Of these simulated plant types the petiole lengths per rank after 46 days of growth were compared with measured petiole lengths after 46 days of two Arabidopsis genotypes (see Experiments for model validation).

To quantify the impact of variation in plastic response curves on plant performance in competitive settings, and to determine if stronger response curves would result in tragedy of the commons, three additional scenarios were simulated. In these scenarios, mixtures of two plant types, placed in a checkerboard design, and their monocultures, were simulated for 46 days and the organ specific growth, light absorption and total aboveground biomass during the development of the stands were recorded as model output. In Scenario 3, two plant types were only different in their petiole elongation response curve; 'Col-0' having a slope of 0.054 and ' $h f r 1-5$ ' of 0.073 (respectively matching the measured Col-0 and hfr1-5 Arabidopsis genotypes). Simulated total aboveground biomass was compared with total aboveground biomass measured from the validation experiment. In Scenario 4 and 5, two plant types had different hyponastic responses but similar petiole elongation response curves. In Scenario 4 the plants increased their angle with 10 (plant type '10deg') or 15 ('15deg') degrees per time step, whereas in Scenario 5, plant types increased their angle with 30 and 35 degrees per time step (respectively '30deg' and '35deg' plant type). These hyponasty values are chosen based on observed variation in hyponastic values of different Arabidopsis genotypes (see also (de Wit et al., 2012). All model simulations were replicated 
10 times to capture the variation in plant growth created by the stochastic nature of the light model and the random plant rotation angle. The mean value of the middle 16 (monocultures) or 8 (mixtures) individuals per genotype per plot was calculated and used as independent replica for further analysis.

\section{Results}

\section{Variation in the petiole elongation response curve}

The three Arabidopsis genotypes showed a gradually increasing relative petiole elongation with decreasing R:FR (Figure 3.2a). Col-0 and hfr1-5 showed only a marginally different elongation response, where rot3-1 clearly had a lower relative petiole elongation rate at the same R:FR conditions compared to the other two. However, all the fitted curves had distinct slope values for their response curves: 0.054 for Col-0, 0.073 for $h f r 1-5$ and 0.028 for rot3-1. The normalization procedure resulted in three response curves with distinct slopes that all increased with decreasing R:FR ratio (Figure 3.2b).
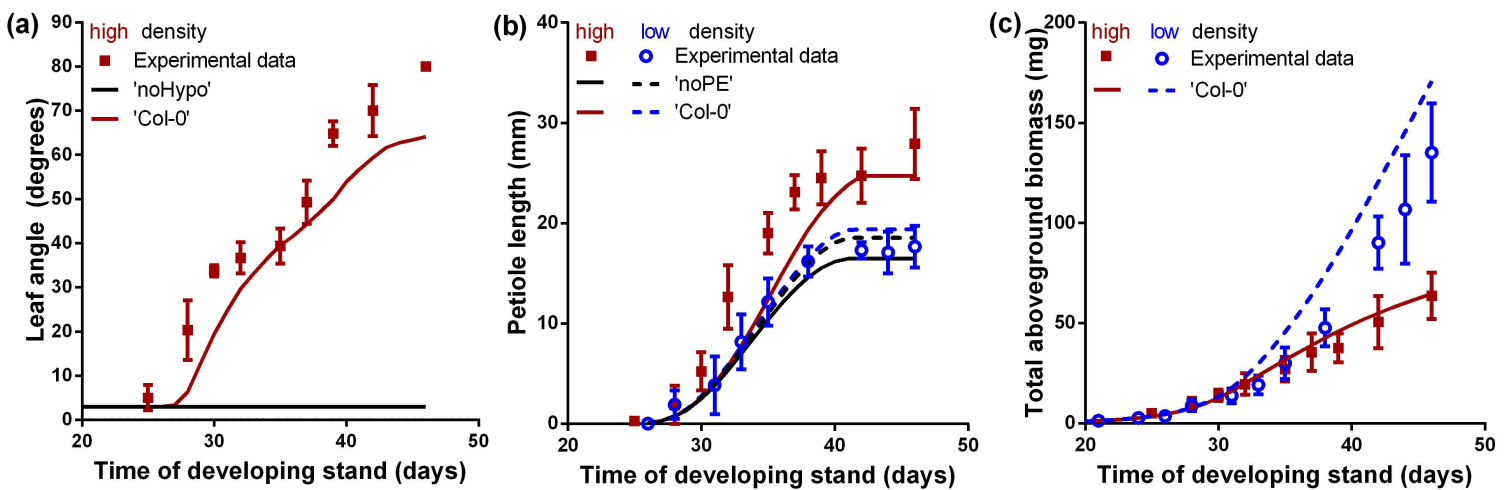

Figure 3.3 Comparison of experimentally obtained phenotypic and plant performance data with data from different simulated plant types to test the model design. (a) Leaf angle during the development of a high-density stand obtained from experimental data (square) or simulated for a plant type that does not show hyponastic responses ('noHypo' - black line) and a plant type that does show hyponastic responses ('Col-0' - red line). Mean of leaf rank number 8 and 10 are illustrated. (b) Petiole length increase during plant development growing in low (open/dotted) and high (solid) density, from experimental data (symbols) and simulated by a plant type that does not show petiole elongation ('noPE' - black line) and a plant type that does show petiole elongation ('Col-0' - red line). Petiole rank number 12 is used and is representative for other leaf ranks. (c) Total aboveground biomass during plant development growing in low (open/dotted) and high (solid) density stands, from experimental data (symbols) and simulated by the default plant type 'Col-O' (lines) that included both hyponastic and petiole elongation responses. Experimental data represent mean \pm SD with $n=10$ for low and $n=18$ for high density). Simulated data represents mean $(n=10)$. 

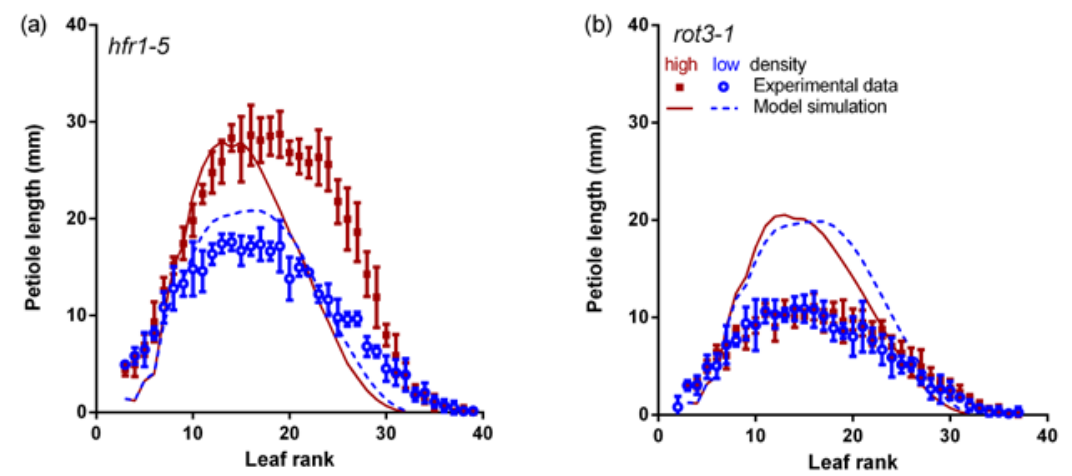

Figure 3.4 Petiole lengths of all leaf ranks per plant after $\mathbf{4 6}$ days of growth of two Arabidopsis genotypes. (a) Petiole lengths of $h f r 1-5$ and (b) rot3-1 plant from experimental data (symbols) or simulated by the model (lines) in two different environments; high (solid red) and low (dotted blue) population density stands. Experimental data represent mean \pm SD with $n=10$ for low and $n=18$ for high density). Simulated data represents mean $(n=10)$.

\section{Test model design (Scenario 1)}

The dynamic change of leaf angle and petiole length of experimentally grown plants in low and high density stands were best simulated by the plant type that included both SAS responses (referred to as 'Col-0') (Figure 3.3). When the hyponastic responses were set to zero ('noHypo'), plants did not become hyponastic in high density compared to the 'Col-0' type, although these simulated 'Col-0' plants increased their leaves slightly later during stand development. Plants that had no petiole elongation response ('noPE') could not grow longer petioles in high density compared to low density, illustrating that the petiole elongation response curve included in 'Col-0' plant type is needed to simulate long petiole lengths in high density population stands. Overall, when including the SAS response values based on wild-type Col-0 ('Col-0'), the model predicted in good agreement the accumulated aboveground biomass during stand development in low and high density stands (Figure 3.3c).

\section{Validate the role of petiole elongation response curve (Scenario 2)}

Validation of the petiole elongation response curve (Scenario 2 ) revealed that the magnitude of the experimentally observed petiole length difference between $h f r 1-5$ plants grown in low or high density stands was predicted by the model that used the ' $h f r 1-5$ ' response curve, although petiole lengths of leaves with high ranks were underestimated (Figure 3.4a). In addition, the model predicted no petiole length difference when using the 'rot3-1' response curve, which is in agreement with the experimentally observed petiole lengths of rot3-1 
plants grown in low or high density stands (Figure 3.4b). In absolute terms the model overestimated petiole lengths due to the higher constitutive growth of the simulated Arabidopsis plants compared to the natural rot3-1 plants.

Test the impact of variation in plastic response curves on plant performance (Scenarios 3-5) 'Col-0' and ' $h f r 1-5$ ' plant types had different simulated aboveground biomass after grown 46 days together in a mixture but not when simulated separately in monocultures (Scenario 3; Figure 3.5a). This difference of plant performance in monocultures compared to mixtures was also observed in the experimental data with respectively Col-0 and $h f r 1-5$ Arabidopsis genotypes (Figure $3.5 b)$. In this scenario, the ' $h f r 1-5$ ' type had slightly longer petioles than 'Col-0' both in the monocultures and mixture, but the laminas of ' $h f r 1-5$ ' absorbed more PAR than 'Col-0' only in the mixture (Figure 3.6a,b). The higher PAR absorption at the individual lamina level resulted in higher simulated whole-plant PAR absorption for ' $h f r 1-5$ ' compared to 'Col-0' in the mixture, whereas in the monocultures there was no difference between the two plant types for lamina or whole-plant PAR absorption (Figure 3.6c). Thus, in direct mixed competition the plant type with the slightly stronger petiole elongation response had higher

(a)

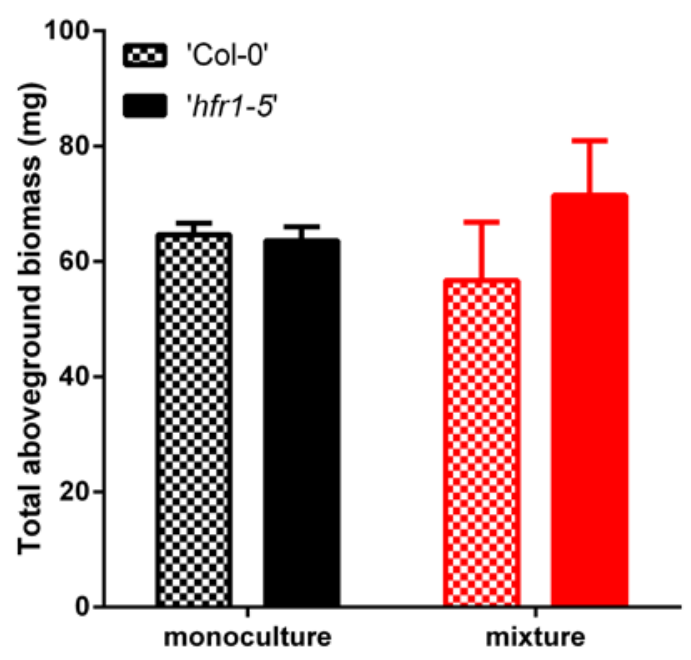

(b) Experimental data

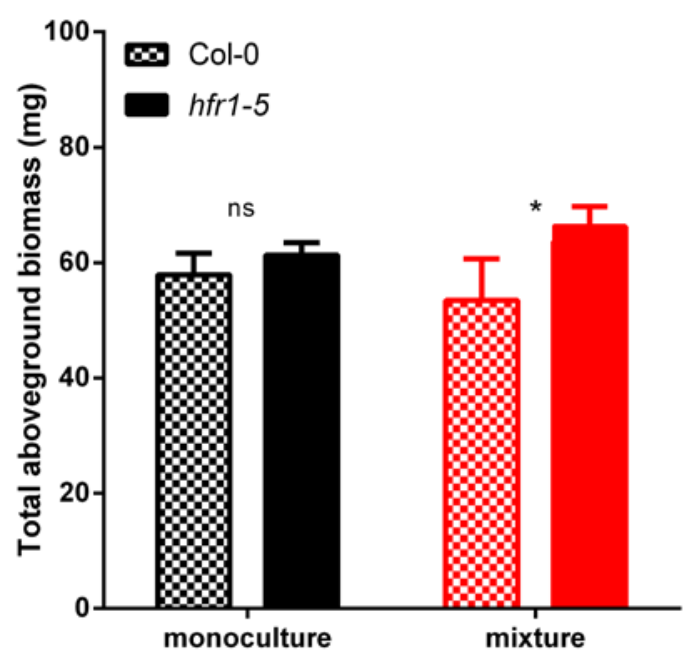

Figure 3.5 Total aboveground biomass of an individual Arabidopsis plant growing in a monoculture or mixture for $\mathbf{4 6}$ days. Plant biomass simulated by the model (a, Scenario 3) or obtained from experimental data (b). Simulated plant types 'Col-0' (dotted) and ' $h f r 1-5$ ' (solid) were based on respectively the Col-0 and hfr1-5 genotypes of Arabidopsis with different slopes for their response curves of respectively 0.054 and 0.073 . Simulated data represents mean $\pm S D(n=10)$. Experimental data represent mean $\pm S D(n=5)$ and $n s ; n o t$ significant and *; $\mathrm{P}<0.05$. 

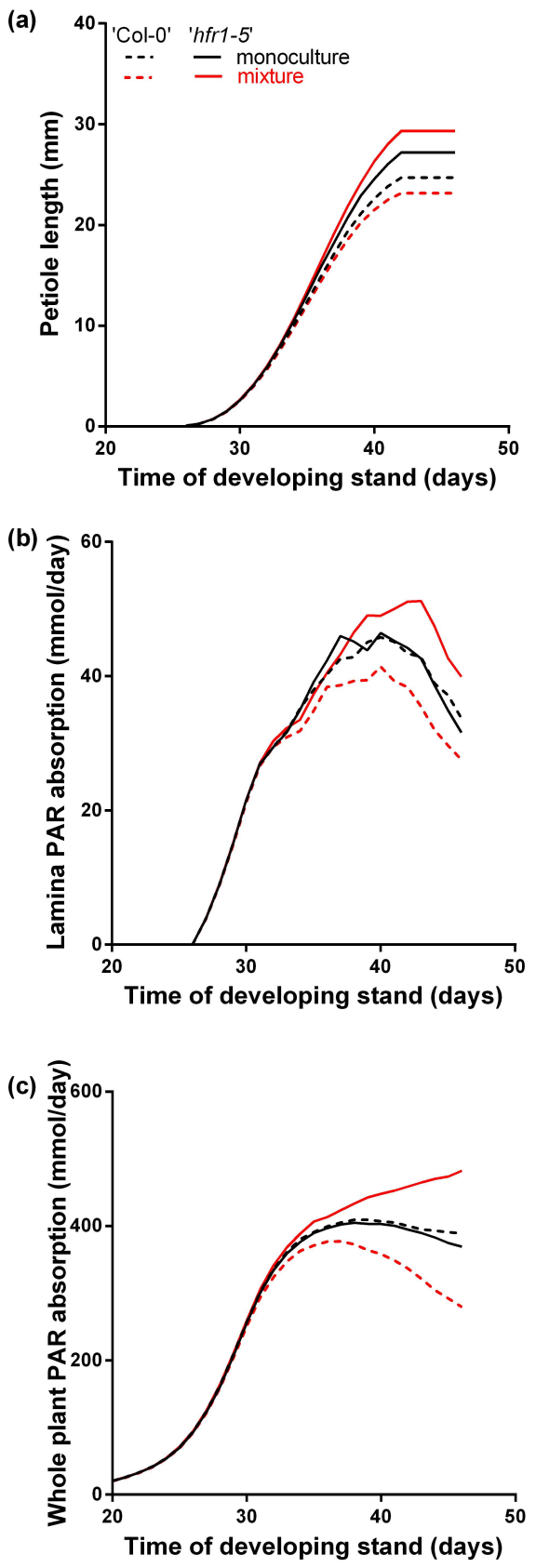

Figure 3.6 Simulated leaf and plant characteristics during the development of Arabidopsis monocultures (black) or mixtures (red) existing by two genotypes with distinct petiole elongation response curves (Scenario 3). ' $h f r 1-5$ ' type (solid line) had a stronger petiole elongation response curve than 'Col-0' type (dotted line), shown in Figure 3.2b. (a) Petiole length, (b) lamina absorbed PAR and (c) whole plant absorbed PAR during Arabidopsis stand development. Leaf rank number 12 is used to visualise petiole length and lamina PAR absorption and is representative for other leaf ranks. performance because it created slightly longer petioles that could put laminas in a better lit part of the canopy.

In the monocultures and the mixture of Scenario 4, in which the strength of the hyponastic response was tested, both plant types showed increased leaf angles at the same developmental stage during stand development, but the '15deg' plant type increased its leaf angle faster (Figure 3.7a). In the mixture, this faster increase resulted in higher lamina PAR absorption that also resulted in higher whole-plant PAR absorption, compared to the weaker '10deg' plant type (Figure 3.7b,c). In the monocultures, the slightly higher leaf angle of the stronger ' $15 \mathrm{deg}^{\prime}$ type did not result in higher lamina or whole plant PAR absorption compared to the '10deg' type. In Scenario 5, the difference in hyponastic response between the two plant types also resulted in a performance difference in the mixture and a similar performance for monocultures (Supporting Information Figure S3.1). However, in contrast to Scenario 4, the plant type with the weaker hyponastic plasticity ('30deg') outcompeted the plant type with the stronger response ('35deg').

\section{Discussion}

In this study we showed that small differences in hyponasty or petiole elongation responses to 
changes in R:FR conditions strongly affect plant phenotype and competitiveness. Model simulations illustrated that subtle variation in SAS response curves influenced competitiveness for light because a small change in a structural trait (petiole length or leaf angle) affected the interaction between plant phenotype and light environment, which directly had consequences for PAR absorption and subsequently growth (Figures 3.6 and 3.7). These model simulations were validated with a plant competition experiment that resulted in biomass accumulation in monocultures and mixture stands for two Arabidopsis genotypes with similar petiole elongation response curves as used in the model simulations that was consistent with model simulations.

\section{Model assumptions}

For the simplicity of the model, only touch and R:FR ratio were the environmental cues that induced the studied SAS responses. It is, however, known that additional canopy-related light cues, especially the decrease of blue and PAR light intensity, are involved in shade avoidance (e.g. reviewed in Casal, 2012; Pierik \& de Wit, 2014) and can strengthen low R:FR responses (de Wit et al., 2016b). In all scenarios, parameters related to leaf optical properties and photosynthesis were set to be independent of light conditions or leaf developmental stage, which is unlike earlier work in which a decrease of the maximal potential of photosynthesis with increased canopy depth is (a) ${ }^{90} 7$ '10deg' '15deg'

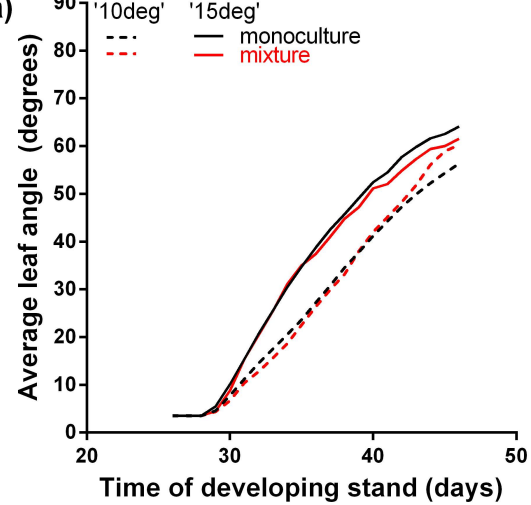

(b)

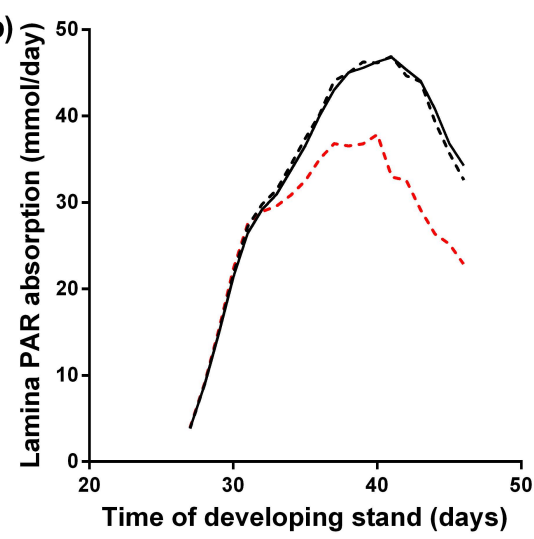

(c)

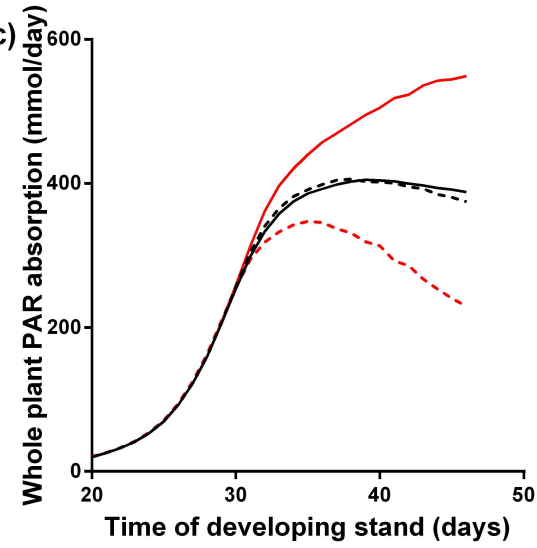

Figure 3.7 Simulated leaf and plant characteristics during the development of Arabidopsis monocultures (black) or mixtures (red) existing by two genotypes with distinct hyponastic responses (Scenario 4). The '15deg' plant type (solid line) had a stronger hyponastic response than the '10deg' plant type (dotted line), respectively 15 and 10 degrees. (a) Leaf angle, (b) lamina absorbed PAR and (c) whole plant absorbed PAR during Arabidopsis stand development. Leaf rank number 12 is used to visualise petiole length and lamina PAR absorption and is representative for other leaf ranks. 
assumed (Anten et al., 1995). We assumed that such acclimations of photosynthetic parameters would be negligible in relative young and quick developed Arabidopsis leaves compared to the role of phenotypic change due to the studied SAS responses. In addition, we assumed that chloroplasts in the petioles contributed to resource harvesting (PAR absorption and photosynthesis). Other light competition models (Anten, 2005; Dybzinski et al., 2011), however, make a clear distinction between height growth through investments in stems and branches that were considered to not contribute directly to $\mathrm{CO}_{2}$ fixation and light harvesting organs (leaves) that do fix carbon. We checked the photosynthetic contribution of petioles, and our model simulations showed that also without petiole photosynthesis plants with a slightly different plastic response curve have different performances in mixture but equal performances in monocultures (Supporting Information Figure S3.2). Modelling the induction of both SAS responses was based on R:FR perception at the lamina (Kozuka et al., 2010). However, details on site of perception versus site of response may differ between species, organs and responses (Casal \& Smith, 1988a,b; Maddonni et al., 2002). The kind of organ level plant modelling presented in this paper makes it possible to explore what would be the environmental context of R:FR distributions and functional implications of localized signalling.

\section{Cost related to plasticity}

The balance between costs and benefits of plant responses to environmental cues is an important determinant of plant competitiveness. In our simulation model we regarded the direct consequences of phenotypic changes for light capture and substrate allocation as costs for expressing plastic responses: Substrates invested in petiole length were consequently not available for lamina growth and inclined leaf angles can potentially absorb less light. Because increased leaf angles can lead to decreased light capture, strong hyponastic responses can have negative consequences for plant performance (illustrated in Figure 3.7 and Figure S3.1). We assumed the relationship between specific petiole length (SPL; petiole length per mass) to be a negative function of petioles length. This implies that biomass costs of petioles increased disproportionately with length, which has progressively negative effect on carbon availability for lamina growth. Other indirect costs, such as vulnerability of strongly hyponastic leaves and long petioles to mechanical damage (e.g. by trampling) or hydraulic limitations, were not taken into account in our model simulations. 
However, these indirect costs may exert a particular selection pressure for dampened SAS responses. Overall, it is worth noting that the model predicted the observed differences in biomass production between genotypes with different petiole elongation responses well (Figure 3.5), suggesting that costs and benefits of the petiole elongation response were reasonably well captured in the current model regarding Arabidopsis responses. In follow-up studies, specific costs of phenotypic plasticity, for example active down regulation of lamina area upon low R:FR perception (de Wit et al., 2015) or the costs of having a sensory machinery to sense environmental conditions (also referred to as maintenance cost of plasticity (Ernande \& Dieckmann, 2004)), or some other degree of indirect cost, could be implemented to further analyse the sensitivity of adaptive significance of plasticity for resource competition.

\section{Tragedy of the commons}

Tragedy of the commons in light competition assumes that plants investing relatively more in light harvesting compared to its neighbour plants are the most successful competitors, but because of the costs associated with this investment, such plants will perform less when growing as monocultures (Rankin et al., 2007). Thus, the tragedy lies in the observation that on the level of monocultures (the commons) plants collectively make investments that cannot pay off since all neighbours act the same way. This conflict between individual based selection and population performance has been proposed to have mayor consequences for vegetation functioning and knowledge of this phenomenon may provide input for crop management and breeding systems (Anten \& Vermeulen, 2016). Contrary to mostly theoretical studies that evaluate tragedy of the commons, our results do not provide evidence for a tragedy of the commons in light competition (see Figure 3.5). In our study the plant type with the stronger petiole response and thus a higher petiole investment, outcompeted the individual with the weaker response in the mixtures but had equal performance in monoculture. This suggests that selection on plastic responses that favour competition does not necessarily result in lower population-level performance. The extent to which these results can be extrapolated to other plant types such as forest trees or crops that often have different growth forms and associated SAS responses than Arabidopsis, still needs to be explored. For instance, costs associated with petiole elongation could be different from those of stem elongation; e.g. failure of the stem that supports the entire 
shoot would be more detrimental than failure of a petiole that supports one lamina. However if the pattern that small genetic difference in SAS responses affect competitive ability without necessarily impacting monoculture performance extends to crops it could provide useful breeding targets. An example in this respect would be competitiveness of crops against weeds.

\section{Promising avenues}

In this study we described plasticity as trait responses to a range of changing environmental conditions during the lifetime of the individual plant. For example, a long petiole after competition in a high population density stand (Figure 3.4 ) was the result of petiole growth rates that increased upon the perception of decreasing R:FR ratios during stand development, compared to a short petiole in low population density stands in which R:FR barely decreased during stand development. Differences in plastic responses were described by different shapes of the response curves (Figure 3.2), and these differences in response curve allowed to quantify how variation in plastic responses would affect plant competitiveness. Describing plasticity as trait responses in time is fundamentally different from describing plasticity as absolute differences in trait values in various densities and test the adaptive significance of those absolute trait values (Schmitt et al., 1995; Dudley \& Schmitt, 1996; Weijschede et al., 2008). In addition, describing the level of plasticity with a single parameter and regarding variation in this single parameter as variation in the level of plasticity is required to analyse how natural selection could have acted the level plasticity in plants. Analysing how natural selection could have acted on trait values has often been approached by using game theoretical models (reviewed by Parker \& Maynard Smith, 1990; Falster \& Westoby, 2003; McNickle \& Dybzinski, 2013). However, analysing selection for plasticity is challenging because a model system is required that considers i) the possibility of a single genotype to express multiple phenotypes, ii) the dynamic interaction between phenotypic changes and changes in environmental conditions and iii) variation in plasticity should be incorporated by a single parameter. The model system presented here complies with these three requires. In that manner it builds on previous game theoretical studies (Dybzinski et al., 2013; Farrior et al., 2013; Vermeulen, 2014) that considered plasticity but focussed on absolute trait values expressed in different environments rather than dynamic 
trait responses as was done here. We thus argue that our approach provides a useful complementary approach to analyse natural selection for plasticity (Bongers et al., 2014).

\section{Conclusion}

In this paper we illustrated that substantial difference in competitiveness may arise between phenotypes with only marginally different SAS response levels, due to the amplification of plant growth differences by small changes in plant phenotype. Therefore we conclude that a strong selection pressure is likely to have played a role in fine-tuning the sensitive SAS responses found in plants and that competitive strong fine-tuned responses do not necessarily have to result in tragedy of the commons.

\section{Acknowledgements}

We thank J. de Vries for help with model development and M.F. Fouce Fernández and members of the UU Plant Ecophysiology team for help with harvesting plant experiments. Funding was provided by the Netherlands Organisation for Scientific Research (ALW grant no. 821.01.014 to N.P.R.A, VIDI grant no. 86412.003 to R.P). 


\section{Supporting Information}

Table S3.1 Overview of all used parameters in the FSP model of Arabidopsis, with parameter description, unit, value and source of parameter value.

\begin{tabular}{|c|c|c|c|c|}
\hline PARAMETER & DESCRIPTION & UNIT & VALUE & LITERATURE \\
\hline \multicolumn{5}{|c|}{ Substrates and growth } \\
\hline$A_{\max }$ & $\begin{array}{l}\text { maximal photosynthesis } \\
\text { rate }\end{array}$ & $\mu \mathrm{mol} \quad \mathrm{CO}_{2} \mathrm{~m}^{-2} \mathrm{~s}^{-1}$ & 18 & Pons 2012 \\
\hline epsi & light efficiency & & 0.08 & Evers et al. 2010 \\
\hline $\mathrm{R}_{\mathrm{d}}$ & dark respiration & - & 1.2 & Evers et al. 2010 \\
\hline MCO2 & molar mass of $\mathrm{CO} 2$ & $\mathrm{~g} \mathrm{~mol}^{-1}$ & 44 & Evers et al. 2010 \\
\hline $\mathrm{fCO} 2$ & carbon mass fraction & - & 0.8 & Evers et al. 2010 \\
\hline $\mathrm{Rm}$ & maintenance respiration & - & 0.015 & Evers et al. 2010 \\
\hline $\mathrm{R}_{\mathrm{g}}$ & growth respiration & - & 0.195 & Chew et al. 2014 \\
\hline endosperm & seed substrates & $\mathrm{mg}$ & 0.02 & Bongers, unpublished \\
\hline RS & root:shoot ratio & - & 0.2 & Bongers, unpublished \\
\hline \multicolumn{5}{|l|}{ Light } \\
\hline Rref & reflectance $\mathrm{R}$ & - & 0.075 & de Wit et al. 2012 \\
\hline FRref & reflectance FR & - & 0.492 & de Wit et al. 2012 \\
\hline PARref & reflectance PAR & - & 0.103 & de Wit et al. 2012 \\
\hline Rtrans & transmittance $\mathrm{R}$ & - & 0.042 & de Wit et al. 2012 \\
\hline FRtrans & transmittance FR & - & 0.377 & de Wit et al. 2012 \\
\hline PARtrans & transmittance PAR & - & 0.073 & de Wit et al. 2012 \\
\hline Rabs & absorbance $\mathrm{R}$ & - & 0.883 & de Wit et al. 2012 \\
\hline FRabs & absorbance FR & - & 0.131 & de Wit et al. 2012 \\
\hline PARabs & absorbance PAR & - & 0.824 & de Wit et al. 2012 \\
\hline \multicolumn{5}{|c|}{ Plant structure } \\
\hline plastochron & $\begin{array}{l}\text { time between successive } \\
\text { leaves }\end{array}$ & day & $\begin{array}{l}\text { rank } 1-12 ; 0,0,6,0 \\
3,1,4,2,1,2,2,2 ; \\
\text { else: } 1\end{array}$ & this paper \\
\hline phyllotactic & $\begin{array}{l}\text { angle between successive } \\
\text { leaves }\end{array}$ & degrees & $\begin{array}{l}\text { rank 1-4: } 0,180,90, \\
180 \\
\text { else: } 137.5\end{array}$ & $\begin{array}{l}\text { Mundermann et al. } \\
2005\end{array}$ \\
\hline LWL & length:width ratio lamina & ratio & $\begin{array}{l}\text { rank 1-4: } 1.0 ; \text { rank 5- } \\
6: 1.4 \text {; else: } 1.8\end{array}$ & this paper \\
\hline LWP & length:width ratio petiole & ratio & 7.9 & this paper \\
\hline PWmax & maximal petiole with & $\mathrm{cm}$ & 0.2 & this paper \\
\hline SLA & specific lamina area & $\mathrm{cm}^{2} \mathrm{mg}^{-1}$ & $\begin{array}{l}\text { rank 1-2: } 0.80 \text {; else: } \\
0.68\end{array}$ & this paper \\
\hline WmaxL & $\begin{array}{l}\text { maximal potential lamina } \\
\text { area }\end{array}$ & $\mathrm{cm}^{2}$ & rank 1-2: 1.5; else: 3 & this paper \\
\hline WmaxP & $\begin{array}{l}\text { maximal potential petiole } \\
\text { length }\end{array}$ & $\mathrm{cm}$ & 2 & this paper \\
\hline TeL & growth duration lamina & age & 22 & this paper \\
\hline TeP & growth duration petiole & age & 17 & this paper \\
\hline $\mathrm{TmL}$ & $\begin{array}{l}\text { lamina age at which } \\
\text { growth rate is maximal }\end{array}$ & age & 4 & this paper \\
\hline
\end{tabular}




\begin{tabular}{|c|c|c|c|c|}
\hline $\mathrm{TmP}$ & $\begin{array}{l}\text { petiole age at which } \\
\text { growth rate is maximal }\end{array}$ & age & rank 1-4: 4; else: 10 & this paper \\
\hline senescence & $\begin{array}{l}\text { Leaf age for leaf } \\
\text { senescence }\end{array}$ & age & 40 & this paper \\
\hline SPLmax & $\begin{array}{l}\text { maximal specific petiole } \\
\text { length }\end{array}$ & $\mathrm{cm} \mathrm{mg}^{-1}$ & rank 1-4: 10; else: 4 & this paper \\
\hline SPLmin & $\begin{array}{l}\text { minimal specific petiole } \\
\text { length }\end{array}$ & $\mathrm{cm} \mathrm{mg}^{-1}$ & 0.1 & this paper \\
\hline SPLk & $\begin{array}{l}\text { slope of the relation } \\
\text { between specific petiole } \\
\text { length and petiole length }\end{array}$ & - & 0.2 & this paper \\
\hline
\end{tabular}
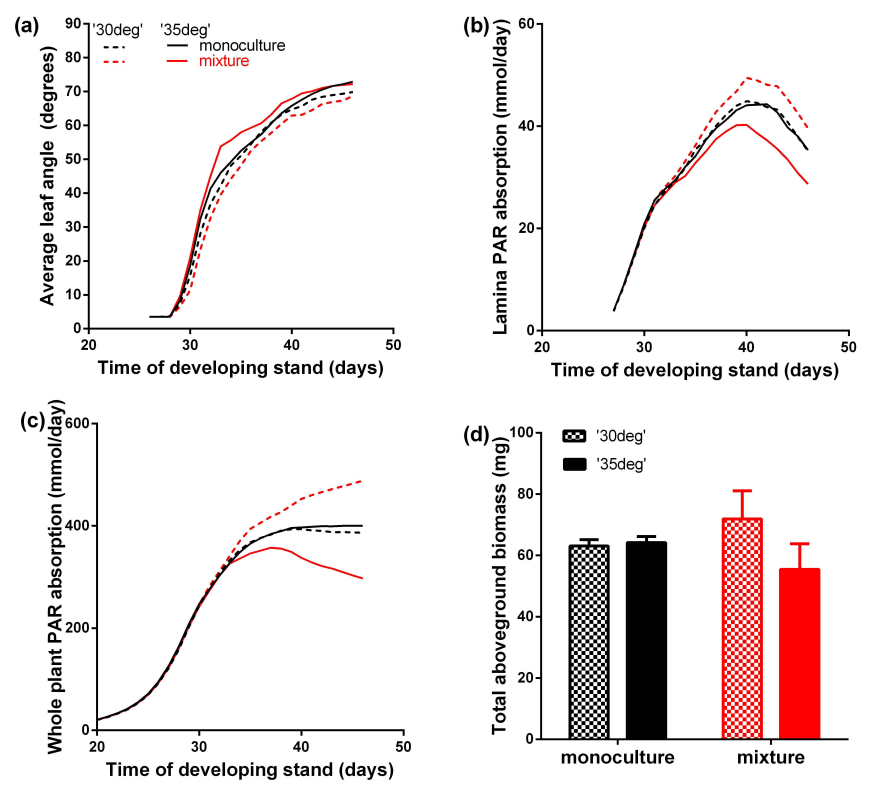

Figure S3.1 Simulated leaf specific and whole plant characteristics of an individual plant during the development of Arabidopsis monocultures (black) or mixtures (red) existing by two genotypes with distinct hyponastic responses. The '35deg' plant type (solid line) had a stronger hyponastic response than the '30deg' type (dotted line), respectively 35 and 30 degrees per hyponastic event. (a) Leaf angle, (b) lamina absorbed PAR and (c) whole plant absorbed PAR during the development of the Arabidopsis stands. For leaf specific characteristics leaf rank number 12 is visualised and is representable for other leaf ranks. (d) Total aboveground biomass after 46 days of simulated growth for the '30deg' and '35deg' plant types in monoculture and mixture. Data represents mean $\pm S D(n=10)$.

Figure S3.2 Simulated total aboveground biomass of an individual Arabidopsis plant growing in monoculture or mixture for $\mathbf{4 6}$ days. Similar model simulations are simulated as in Scenario 3 and 4, but in this two scenarios the petioles were not able to photosynthesize and thus did not contribute to total amount of substrates for growth. (a) Simulated 'Col-0' and ' $h f r 1-5$ ' plant types had similar hyponastic responses but different petiole elongation responses, see Figure 3.2. (b) Simulated '10deg' and '15deg' plant types had similar petiole elongation responses but different hyponastic responses, respectively 10 and 15 degrees per hyponastic event. Data represents mean \pm SD $(n=10)$. 

CHAPTER 4

\title{
Tissue specific R:FR perception induces different adaptive plastic responses
}

\author{
Franca J Bongers $^{1,2, \#}$, Chrysoula K Pantazopoulou ${ }^{2, \#}$, Jesse J Küpers², \\ Jochem B Evers ${ }^{1}$, Niels PR Anten ${ }^{1}$, Ronald Pierik ${ }^{2}$ \\ ${ }^{*}$ these authors contributed equally \\ ${ }^{1}$ Centre for Crop Systems Analysis, Wageningen University, Wageningen, the Netherlands. \\ ${ }^{2}$ Plant Ecophysiology, Utrecht University, Utrecht, the Netherlands.
}




\section{Abstract}

Vegetation stands have a heterogeneous light quality distribution, including the red : far-red light ratio (R:FR) that informs plants about proximity of neighbours. Adequate responses to changes in R:FR are important for competitive success. How the detection and response to $R: F R$ are spatially linked and how this spatial coordination between the two affects plant performance remains unresolved. We show in Arabidopsis thaliana and Brassica nigra that local low R:FR perception at the petiole induces petiole elongation and that low R:FRperception at the lamina tip induces upward leaf movement (hyponasty). With organ-level physiology we show that auxin is involved in hyponasty, which results from a special separation of side of R:FR detection and response. By using computational 3D modelling we show that using R:FR perception at the petiole and lamina tip can both be beneficial during competition for light when used to induce petiole elongation and hyponasty, respectively. Lamina tip perception for hyponasty has an adaptive advantage over petiole perception in the petiole, since it optimizes timing of upward leaf movement in responses to neighbour proximity. Insight regarding organ-specific induced phenotypic responses could help the each for crop ideotypes, specifically in greenhouse horticulture where additional lighting can be applied. 


\section{Introduction}

In plant canopies the light intensity differs by up to two orders of magnitude between the top and bottom of vegetation stands, because leaves shade one another (e.g. Monsi et al., 2005). Due to clustering of leaves, light intensities also vary horizontally. As light is essential for growth this creates selection pressure for plants to position their leaves for optimal light capture, preferably before their neighbours do so. Leaves do not absorb all wavelengths of the incoming light equally, and so quality of light also differs both vertically and horizontally in canopies (Monsi et al., 2005; Boonman et al., 2006, 2009; Crepy \& Casal, 2015) and even across the surface of single leaves (Chelle et al., 2007). Leaves preferentially absorb red (R, $\lambda=600-700 \mathrm{~nm})$ and blue $(B, \lambda=400-500 \mathrm{~nm})$ light for photosynthesis, whereas they reflect and transmit most of the far-red (FR, $\lambda=700-800 \mathrm{~nm}$ ) light. This leads to a relative enrichment of FR light (a low R:FR ratio) in the local vicinity of leaves. Low R:FR therefore provides a signal of neighbour proximity (Holmes \& Smith, 1977; Ballaré et al., 1987).

Low R:FR is sensed by phytochrome photoreceptors, mainly phytochrome B (phyB), complemented at later stages by cryptochrome-mediated detection of blue light depletion (Casal, 2013; Pierik \& de Wit, 2014; Fraser et al., 2016). Upon neighbour detection plants promote upward leaf movement (hyponasty) and elongation of stems and petioles. These traits contribute towards the shade avoidance syndrome (SAS) and bring the leaves higher towards the more illuminated parts of the canopy (reviewed in Franklin, 2008; Casal, 2013; Pierik \& de Wit, 2014). Plants are modular organisms and SAS responses are thought to be restricted to the specific modules that sense shade cues (de Kroon et al., 2005; Ballaré \& Pierik, 2017). However, given the heterogeneity in the light climate, the magnitude and timing of a response are strongly determined by the integration of cue detection and response. Whether cue detection and SAS responses are locally linked (they occur in the same plant part) or separated (detection being in a different part of the plant) and how it is regulated, has not been intensively studied. Recent studies in very young seedlings indicate that cotyledons sense R:FR to control elongation of the hypocotyl (Kozuka et al., 2010; Procko et al., 2014), however little is known about these spatial aspects in relatively mature plants that are large enough to experience light quality heterogeneity over the plant body. In addition, it was shown recently in Brassica rapa that auxin biosynthesis occurs in the 
cotyledons, and transport to the hypocotyl subsequently induces cell elongation in low R:FR (Procko et al., 2014). Also in Arabidopsis, low R:FR-induced hypocotyl elongation relies on auxin transport (Pierik et al., 2009) through among others the PIN3 auxin efflux-associated protein (Keuskamp et al., 2010). In analogy to the cotyledon-hypocotyl division of auxin dynamics (Procko et al., 2014), it was recently proposed by de Wit et al., (2015) that a similar division may exist within a single leaf. Among other these studies propose the involvement of auxin transport in low R:FR induced responses.

We hypothesize that a spatial separation of R:FR detection may allow plants to deal with the spatially heterogeneous light climate in dense stands where R:FR responses are functional. Therefore we investigated leaf movement and elongation responses to local low R:FR conditions. To determine the adaptive significance of tissue specific perception and response, a functional-structural plant (FSP) model was used (principles reviewed in Vos et al., 2010). These FSP models scale from organ to whole-plant level and can explicitly simulate competition for light (Evers \& Bastiaans, 2016). As light absorption, perception and responses occur at the organ-level and drives whole-plant growth, organ-level light responses can be quantified for whole-plant phenotype and performance in various canopy structures (Gautier et al., 2000; Evers et al., 2007; Kahlen \& Stützel, 2011; Bongers et al., 2014).

\section{Material and Methods}

\section{Plant growth and measurements}

Arabidopsis wild-type Col-0 seeds were sown on Primasta ${ }^{\circledR}$ soil and stratified for 3 days (dark, $4^{\circ} \mathrm{C}$ ), before transfered to short day (9 h light / $15 \mathrm{~h}$ dark) growth rooms (130-135 $\left.\mu \mathrm{mol} \mathrm{m} \mathrm{m}^{-2} \mathrm{~s}^{-1} \mathrm{PAR}, \mathrm{R}: \mathrm{FR} 2.3,20^{\circ} \mathrm{C}, 70 \% \mathrm{RH}\right)$. After 11 days, seedlings were transplanted to individual $70 \mathrm{ml}$ pots. Twenty eight days old plants were used for experiments; the fifthyoungest leaf was measured for petiole angle and length treatments and measurements. Pictures were taken before $(t=0 \mathrm{~h})$ and after treatment $(\mathrm{t}=24 \mathrm{~h})$ and angles were measured digitally using image-J software. Petiole lengths were recorded with a digital calliper. All experiments started at 10:00 in the morning (Zeitgeber time $=2 \mathrm{~h}$ ). 


\section{Light treatments}

In addition to control white (W) light from Philips HPI lamps (R:FR $=2.3$ ), far-red supplementation treatments were used: whole-plant FR enrichment $\left(W+F R_{\text {whole }}\right)$ by adding supplemented FR LEDs (Philips Green Power FR $730 \mathrm{~nm}$ ) to the W background (resulting in $R: F R=0.05)$, and localized FR enrichment by adding FR spots ( $\varnothing 3.5 \mathrm{~mm})$ to selective parts of the leaf depending on experiment (Supporting Information Figure S4.1a), using custom FR LEDs (724-732 $\mathrm{nm}$ ) on flexible arms, achieving locally a R:FR of 0.05. PAR was unaffected for all treatments, around $125-135 \mu \mathrm{mol} \mathrm{m} \mathrm{m}^{-2} \mathrm{~s}^{-1}$. SpectroSense2-Skye instrument were used for the R:FR measurements and OceanOptics JAZ instrument for PAR and full spectrum measurements. Light spectra are available in Supporting Information Figure S4.1b-d.

\section{Pharmacological treatments}

A droplet of $5 \mu$ of $30 \mu \mathrm{M}$ Indole 3-acetic acid (IAA, Duchefa Biochemie, Haarlem, The Netherlands) per leaf was used to induce auxin signaling, while $5 \mu \mathrm{l}$ of $50 \mu \mathrm{M}$ naphthylphthalamic acid (NPA, Duchefa Biochemie, Haarlem, The Netherlands) was used to block polar auxin transport at the lamina tip or $4 \mu \mathrm{l}$ at the lamina-petiole junction. The IAA and mock solutions contained 0,1\% tween and 0,03\% DMSO and the NPA solution contained $0,1 \%$ tween and $0,05 \%$ DMSO. When NPA was applied to the lamina-petiole junction, the solution was supplemented with $0.05 \%$ agar to ensure that the NPA treatment was accurately applied to the junction. The solutions were applied to their specific location (adaxial side of the lamina tip or lamina-petiole junction) right before commencing the light treatments.

\section{Functional-structural plant model}

A functional-structural plant (FSP) model of Arabidopsis rosettes recently developed (Chapter 3) was used to simulate artificial Arabidopsis plant types, using the simulation platform GrolMP and its radiation model (https://sourceforge.net/projects/groimp/). The model is available upon request. Arabidopsis rosettes are represented by a collection of leaves (represented by a petiole and a lamina) whose appearance rate and shape were based on empirical data. We defined the lamina tip to represent $7 \%$ of total lamina area at the most distal part of the lamina (see Figure 4.6a and Figure 4.7b). In our simulations, the light source emitted PAR, R and FR light, with a R:FR ratio of 2.3. In each model time step, 
these light rays were reflected, transmitted and absorbed by the petioles and laminas according to their wavelength-specific spectral properties. The leaves individually grew in time in three dimensions based on leaf-level PAR absorption, photosynthesis and betweenorgan carbon allocation principles (explained in more detail in Evers \& Bastiaans, 2016). Simulated plant growth depended on the level of competition for light that individual plants experienced with neighbouring plants. Plant size and biomass were therefore an emergent property of the model. Hyponasty was calculated each time step by a unit-step response curve: when R:FR perception at the specific petiole or lamina tip (see Model scenarios) was below a threshold of 0.5 , the leaf angle increased with a fixed amount of 20 degrees,

(a)
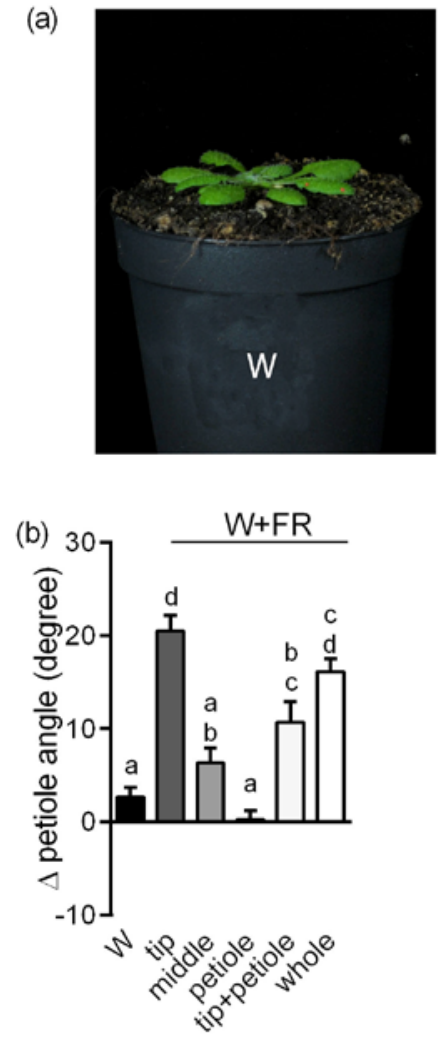
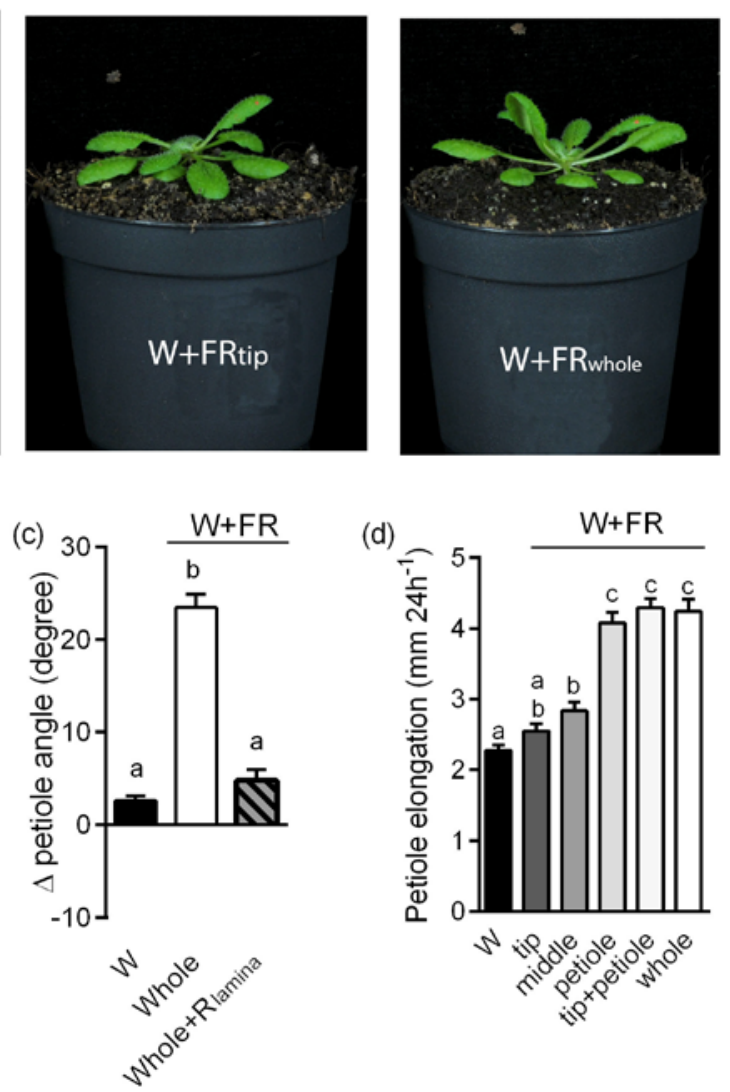

(d)

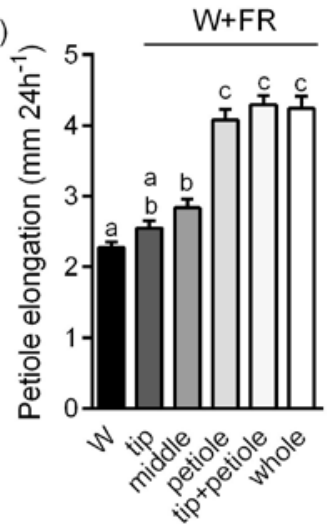

Figure 4.1. Local low R:FR treatment can induce different SAS responses which varies in magnitude depending on the illuminated leaf part. (a) Representative photographs of Col-0 plants treated with white light $(W)$, white-light with supplemented $F R$ light to the lamina tip $\left(W+F R_{\text {tip }}\right)$ and white light with supplemented FR to the whole plant $\left(\mathrm{W}+\mathrm{FR}_{\text {whole }}\right)$. (b) Differential petiole angle for plants exposed to $24 \mathrm{~h}$ white-light control conditions (W) and to supplemented FR light to the lamina tip (tip), lamina middle (middle), the entire petiole (petiole), lamina tip plus petiole (tip+petiole) and the whole plant (whole). (c) Differential petiole angle after 24 $h$ exposure to $W, W+F R_{\text {whole }}$ and $W+F R_{\text {whole }}$ with supplemented $R$ light at the lamina $\left(W+F R_{\text {whole }}+R_{\text {lamina }}\right)$. (d) Petiole elongation response to $24 \mathrm{~h}$ exposure of similar light treatments. Data represents mean $\pm \mathrm{SE}(\mathrm{n}=7-10)$. Different letters represent statistically significant difference (one-way ANOVA with Tukey's Post-hoc test, $\mathrm{p}<0.05$ ). 
otherwise leaf angle was maintained. The angle of the leaf over time was therefore a function of the number of time steps in which this low R:FR perception occurred, with a maximum leaf angle of 80 degrees. Petiole elongation was also calculated each time step by using the response curve described in Chapter 3 (Figure 3.2); relative petiole elongation was induced by R:FR perception at the petiole or lamina tip (see Model scenarios). Organs could only induce hyponasty and petiole elongation during the actual growth phase.

Small adjustments were made in the model used for this study compared to the model described in Chapter 3. (i) Only leaf laminas and not petioles were considered capable of photosynthesis, because most light competition models make clear distinction between investment in height with organs that do not fix carbon and investment in light-harvesting organs that do. Currently there is no conclusive data about petiole photosynthesis for Arabidopsis. (ii) Touch-induced hyponasty was not included to emphasise the role of R:FR induced hyponasty during light competition. (iii) Lamina carbon demand was slightly increased to reduce the creation of reserves that were not involved in virtual plant structures.

\section{Model scenarios}

All Arabidopsis stand types were constructed by placing $4 \times 4$ plants with different interplant distances $(1,2,5$ and $10 \mathrm{~cm})$ resulting in four population densities; 10 000, 2500, 400, 100 plants $\mathrm{m}^{-2}$. In stands with low density (100 plants $\mathrm{m}^{-2}$ ) neighbouring plants did not interact with each other, and no additional rows were simulated. Stands with a density of 400 and 2500 plants $\mathrm{m}^{-2}$ were simulated with two extra plant rows and stands with extreme high density (10 000 plants $\mathrm{m}^{-2}$ ) with four extra plant rows to correct for possible border effects. Plant orientations at their positions in the field were random, just like would be the case in experimental plots. In the first simulation scenario, two stands were simulated at a density of 100 and 2500 plants $\mathrm{m}^{-2}$, representing low and high density, with plants that did not use the incident R:FR for signalling and therefore did not induce hyponasty or petiole elongation. This scenario was used to quantify R:FR on tips and petioles at different leaf ranks in non-responsive plants. The second and third scenarios were done to assess the importance of separation of site of R:FR perception and site of response for plant competitiveness. In these scenarios, mixed stands were simulated at four densities. In these 

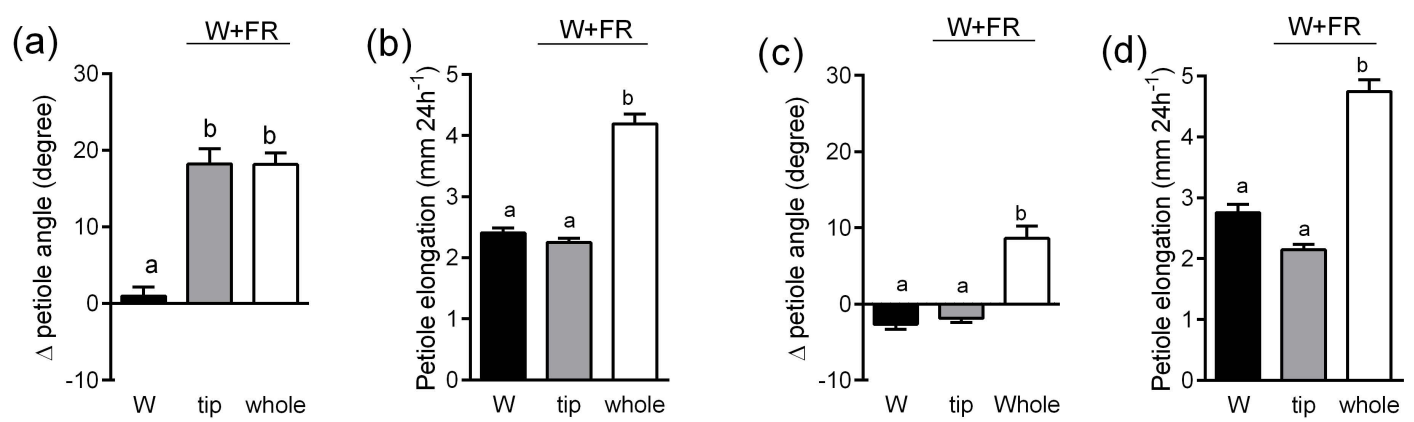

Figure 4.2. Low R:FR treatment does not induce hyponasty or petiole elongation in systemic leaves. (a) The differential petiole angle and (b) petiole elongation of the target leaf in $W, W+F R_{\text {tip }}$, and $W+F R_{\text {whole }}$ condition. (c) The differential petiole angle and (d) petiole elongation of the systemic leaf under the same light conditions. Data represents mean $\pm S E(n=10)$. Different letters indicate statistically significant differences (one-way ANOVA with Tukey's Post-hoc test , $\mathrm{p}<0.05)$.

mixtures, two different plant types were placed in a $50-50 \%$ checkerboard design; the 'Petiole' plant type used the petiole and the 'Tip' plant type used the lamina tip as R:FRdetection organ to induce hyponasty (Scenario 2) or petiole elongation (Scenario 3). In Scenario 2, petiole elongation was equal for both plant types and based on petiole R:FR perception, and in Scenario 3 hyponasty was equal for both types based on lamina tip R:FR perception. Model output was based on the mean of 10 replicated stands, in which first the mean per plant type per stand was calculated.

\section{Results}

\section{Site of R:FR perception determines hyponasty versus petiole elongation}

To study which part of the leaf senses low R:FR for the induction of petiole responses, we illuminated specific regions of Arabidopsis thaliana leaves with FR irradiation spotlight of 3.5 mm (Supporting Information Figure S4.1a) and measured the effect on petiole angle and elongation. Supplemental FR light was given to the lamina tip $\left(W+F R_{\text {tip }}\right)$, lamina middle $\left(\mathrm{W}+F R_{\text {middle }}\right)$, petiole $\left(\mathrm{W}+F R_{\text {petiole }}\right)$, or petiole and lamina tip simultaneously $\left(W+F R_{\text {petiole+tip }}\right)$. As an additional control we included plants that were entirely in supplemental FR $\left(\mathrm{W}+\mathrm{FR}_{\text {whole }}\right)$. We observed that supplemental FR light could induce hyponasty from both the tip and middle of the lamina, whereas treatment of the petiole itself did not elicit any hyponasty at all (Figure 4.1a,b). Importantly, the hyponastic response to FR-enrichment was 
much stronger when given to the lamina tip than when given to the middle of the lamina. Only the supplemental FR-treated leaf would respond; no systemic response was observed (Figure 4.2). When plants were placed under $W+F R_{\text {whole }}$ treatment and one lamina was treated with an R light bundle, hyponasty was reduced specifically in the treated leaf (Figure 4.1c), further corroborating that the response occurs within one leaf and not in systemic leaves. Although $\mathrm{W}+\mathrm{FR}_{\text {petiole }}$ induced no hyponasty at all, it induced maximal petiole elongation, whereas $W+F R_{\text {tip }}$ did not elicit any petiole elongation (Figure $4.1 d$ ). $W+F R_{\text {petiole }}$ induced similar petiole elongation as $W+F R_{\text {whole }}$ and $W+F R_{\text {petiole+tip }}$ (Figure $4.1 d$ ), indicating that local R:FR perception at the petiole of interest is sufficient to account for all elongation observed.

In Brassica nigra we found similar responses related to the specific site of low R:FR perception; petiole hyponasty was induced in W+FRtip, and petiole elongation was mainly

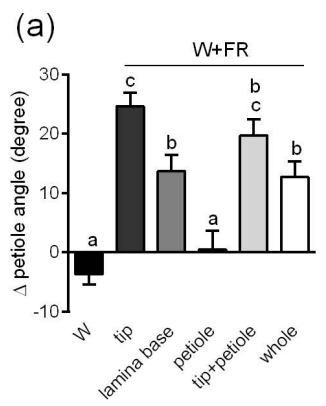

(c)

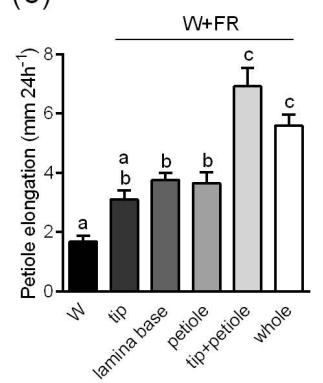

(b)

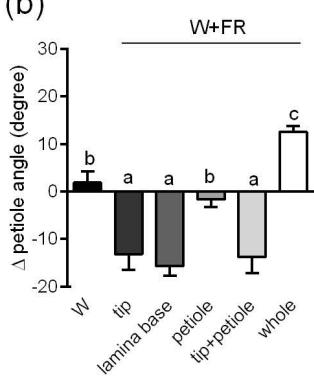

(d)

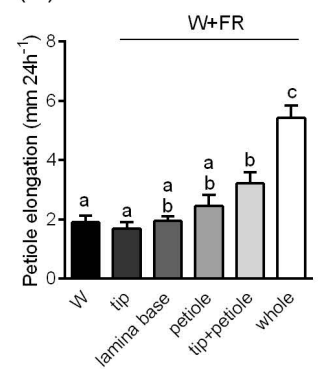

(e)

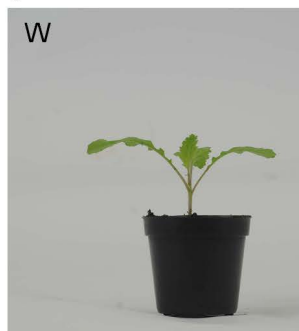

W+FR tip $\checkmark$

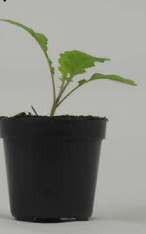

$\mathrm{W}+\mathrm{FR}$ whole

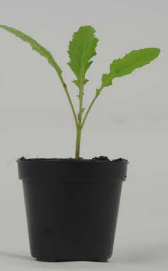

$\mathrm{W}+\mathrm{FR}_{\text {petiole }}$

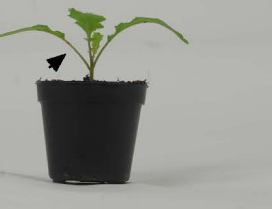

Figure 4.3 Tissue-specific low R:FR perception in Brassica nigra induces hyponastic and elongation responses. Brassica nigra seedlings were grown in $16 \mathrm{~h}$ light $-8 \mathrm{~h}$ dark cycle with 110-150 $\mu \mathrm{mol} \mathrm{m} \mathrm{m}^{-2} \mathrm{~s}^{-1}$ PAR, R:FR 2.3, 20 ${ }^{\circ} \mathrm{C}$ and $70 \% \mathrm{RH}$. The two first leaves of fourteen days old Brassica seedlings were measured for petiole angle and petiole elongation over 24 hours of exposure to different light treatments (cotyledons were removed two days before the treatment). Plants were grown in white light (W) or one leaf (referred to as target leaf) was treated with supplemental FR light applied to the lamina tip (tip), lamina base, petiole, lamina tip plus petiole (tip+petiole) or whole-plant (whole). Petiole angle change of the target leaf (a) and systemic leaf (b), and petiole elongation of the target leaf (c) and systemic leaf (d). Data represents mean $\pm S E(n=10)$. Different letters represent statistically significant difference (one-way ANOVA with Tukey's Post-hoc test, $p<0.05$ ). (e) Representative photographs of the Brassica nigra plants in white light (W), white light with supplemental FR for the whole-plant $\left(\mathrm{W}+\mathrm{FR}_{\text {whole }}\right)$, and in white-light with supplemental $F R$ light at the lamina tip $\left(\mathrm{W}+F R_{\text {tip }}\right)$ or at the petiole $\left(\mathrm{W}+\mathrm{FR}_{\text {petiole }}\right)$ of the left leaf. Black arrows indicate the treated area with supplemental FR for lamina tip and petiole. 
induced by $W+F R$ petiole and only marginally by W+FRtip (Figure 4.3 ), and no systemic induced responses were observed. Summarizing, sensing of low R:FR light via the lamina tip can induce hyponasty but not petiole elongation, while sensing low R:FR light at the petiole induces elongation but not hyponasty.

\section{Auxin regulates responses to low R:FR}

Previous studies illustrated that hyponasty is the result of cell elongation at the abaxial site of the petiole base (Cox et al., 2004; Polko et al., 2012) which indicates that with lamina tip FR-induced hyponasty information has to travel from the lamina tip to the petiole base. Since auxin is the most common hormone known for involvement in signal transport and SAS responses (reviewed in Casal, 2013; Pierik \& de Wit, 2014), we tested the role of auxin for lamina tip induced hyponasty. Local application of the auxin transport inhibitor NPA (N-1Naphtylphthalamic acid) to the lamina tip fully abolished hyponasty when lamina tip and whole plant were treated with supplemental FR (Figure 4.4a). In addition, exogenous application of IAA (Indole 3-acetic acid) at the lamina tip induced hyponasty under W light as well as under low R:FR conditions (Figure 4.4b). However, this IAA-induced hyponasty in W light was abolished when applying the auxin transport inhibiter NPA to the lamina-petiole junction (Figure 4.4c).
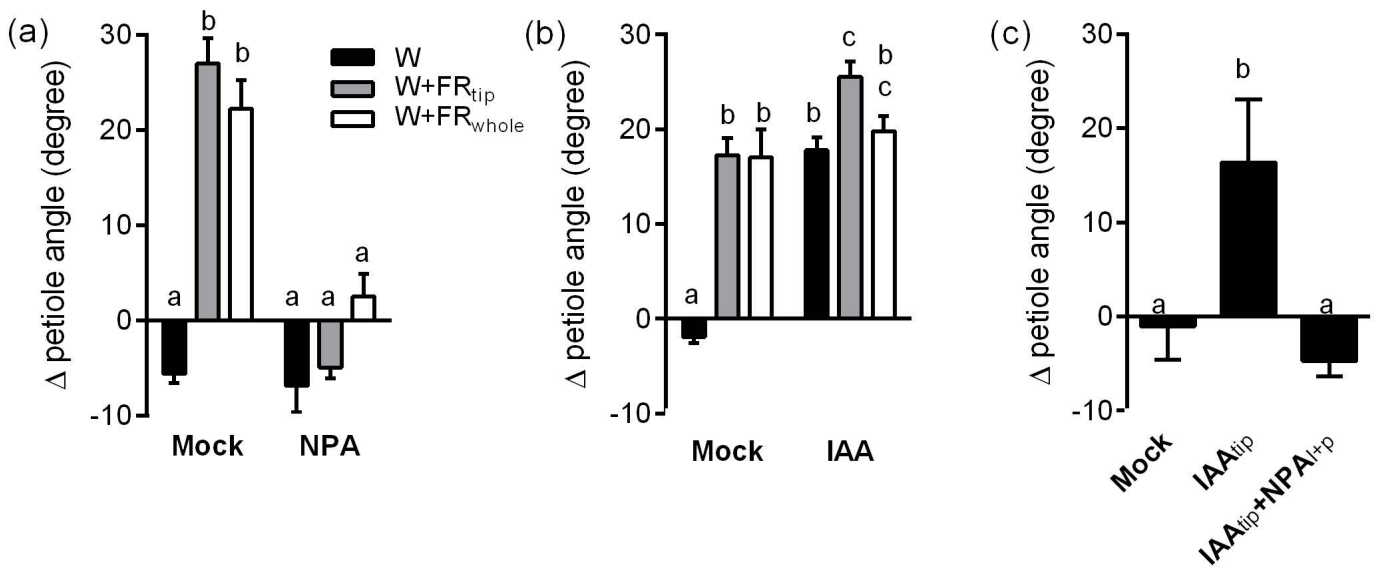

Figure 4.4 Auxin involvement in low R:FR-induced hyponasty. (a) Differential petiole angle in response to white light (W), supplemental FR light to the lamina tip $\left(W+F R_{\text {tip }}\right)$ and supplemental $F R$ to the whole plant $\left(W+F R_{\text {whole }}\right)$ with exogenous application of $50 \mu \mathrm{M}$ NPA or mock to the lamina tip. (b) Differential petiole angle in response to similar light conditions with exogenous application of $30 \mu \mathrm{M}$ IAA to the lamina tip. (c) Differential petiole angle in response to control white light without application of anything (mock), application of $30 \mu \mathrm{M} I A A$ to the lamina tip (IAA tip) or application of $30 \mu \mathrm{M}$ IAA to the lamina tip and $50 \mu \mathrm{M}$ NPA at the lamina-petiole junction $\left(\mathrm{IAA}_{\text {tip }}+\mathrm{NPA}_{1+\mathrm{p}}\right)$. Data represents mean $\pm S E(n=10)$. Different letters indicate statistically significant differences (one-way ANOVA (C) or two-way ANOVE (A, B) with Tukey's Post-hoc test, $p<0.05)$. 


\section{Heterogeneity in R:FR distribution over leaves depends on plant density}

A fully developed and validated FSP model of Arabidopsis rosettes (Chapter 3 ) was used to determine if various tissues (sections) of the leaf experienced different incident R:FR during plant growth (Scenario 1). The model showed that at a low density of 100 plants $\mathrm{m}^{-2}$, where neighbour plants do not interact, petioles get exposed to a decrease of R:FR because of selfshading by newly developed leaves, whereas the lamina tip did not experience a clear change in R:FR (Figure 4.5a). However, when neighbours were present at high density (2500 plants $\mathrm{m}^{-2}$ ), a strong decrease of R:FR at the lamina tip occurred over time, due to leaf overlap by neighbouring plants (Figure 4.5b). Not only the petiole and lamina tip perceived different R:FR ratios during plant development in two different densities; over the whole length of the lamina there was great variation in incident R:FR gradient (Figure 4.6). In high density, the most distal part of the lamina (the tip) experienced the first decrease in R:FR, which was followed by a decrease in R:FR on the rest of the lamina during further canopy development. In low density this pattern was reversed; the lamina only received a low R:FR late in canopy development, but then the section most close to the petiole experienced the first R:FR decrease due to self-shading.

\section{Functional implications of tissue specific R:FR perception}

To evaluate the competitive relevance of R:FR sensing at the petiole or lamina tip, we performed two simulation scenarios of plant competition in $50-50 \%$ checkerboard designs of mixtures of plant s sensing R:FR at the lamina tip (referred to as 'Tip') and plants sensing R:FR at the petiole (referred to as 'Petiole'), at different plant densities. In Scenario 2 the two plant types used the R:FR at either the petiole or lamina tip to induce hyponasty (see illustration for plant types after 46 days of growth at low density in Figure $4.7 a, b)$, and in Scenario 3 the plant types used these organ-level perceptions to induce petiole elongation.

At lower density, neighbour plants are encountered later than at high density and consequently hyponasty was induced later at low density than at high density when R:FR was sensed at the tip (Figure 4.7c and Supporting Information Figure S4.2a). However, when sensing R:FR at the petiole, plants induced hyponasty even at the lowest density where there was no interaction with neighbours (Figure 4.7a,c) and were delayed (compared to the 'Tip' plants) in their response to nearby neighbours at extreme high density (10 000 plants 

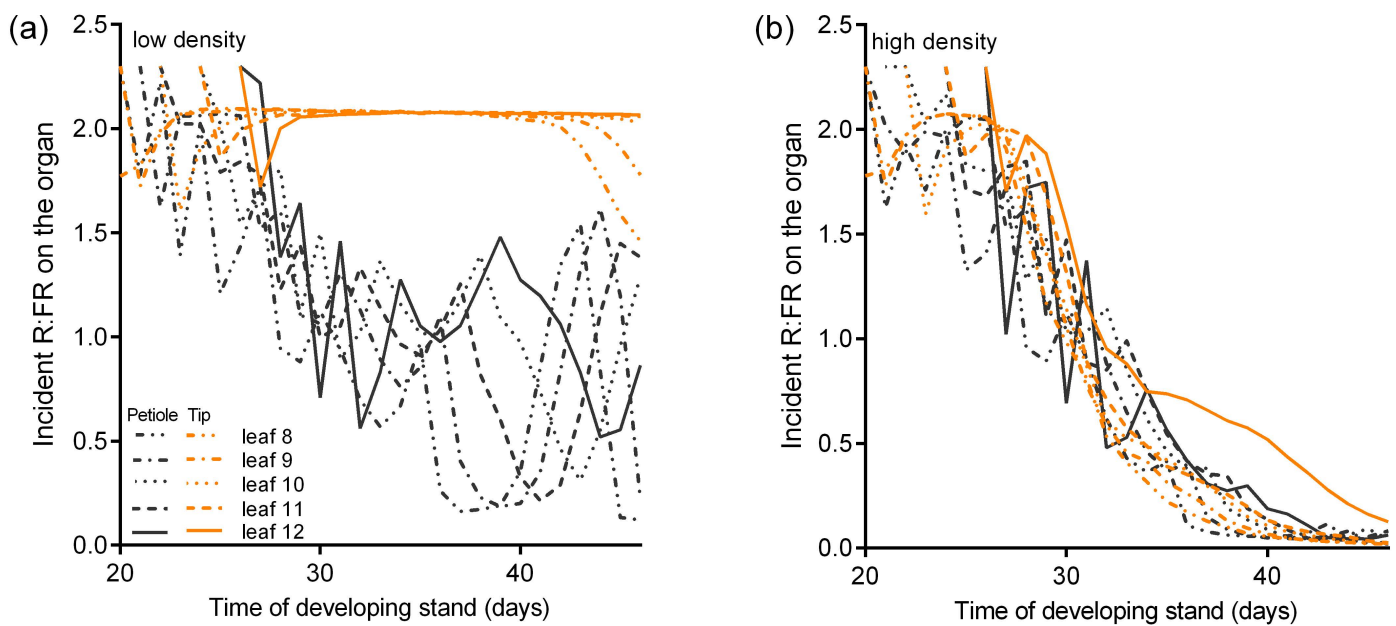

Figure 4.5 Simulated tissue-specific incident R:FR of plants growing in low and high population density (Scenario 1). Incident R:FR at petiole (grey) and tip (orange) of leaf rank 8 up to 12, during plant development growing at low density $\left(100\right.$ plans $\mathrm{m}^{-2}$, a) or at high density $\left(2500\right.$ plants $\left.\mathrm{m}^{-2} ; \mathrm{b}\right)$. In these simulations, plants did not use the R:FR as signal to induce hyponasty or petiole elongation. Data represent mean $(n=10)$.

$\mathrm{m}^{-2}$ ). As a consequence, virtual 'Petiole' plants with petiole R:FR sensing for hyponasty performed less than 'Tip' plants with lamina tip sensing in direct competition (Figure 4.7d). Although decrease of R:FR at the petiole was similar in low and high density (Figure 4.5), virtual 'Petiole' plants created longer petioles than 'Tip' plants that respectively used their petiole and lamina tip to induce petiole elongation (Figure 4.7e and Supporting Information Figure S4.2b). These small differences during canopy development resulted in an overall higher performance for the 'Petiole' compared to the direct 'Tip' competitor (Figure 4.7f). These data indicate that using R:FR perception at the petiole and lamina tip are both beneficial during competition for light when used for the appropriate phenotypic response, i.e. petiole elongation and hyponasty, respectively.

\section{Discussion}

An early plant response to FR light reflected by neighbours is upward leaf movement, called hyponasty, followed by petiole elongation (reviewed in Pierik \& de Wit, 2014). Although light quality distribution in dense stands is heterogeneous even at the level of individual plants, nearly all contemporary shade avoidance studies (but see e.g. Kozuka et al., 2010; Izaguirre 
(a)
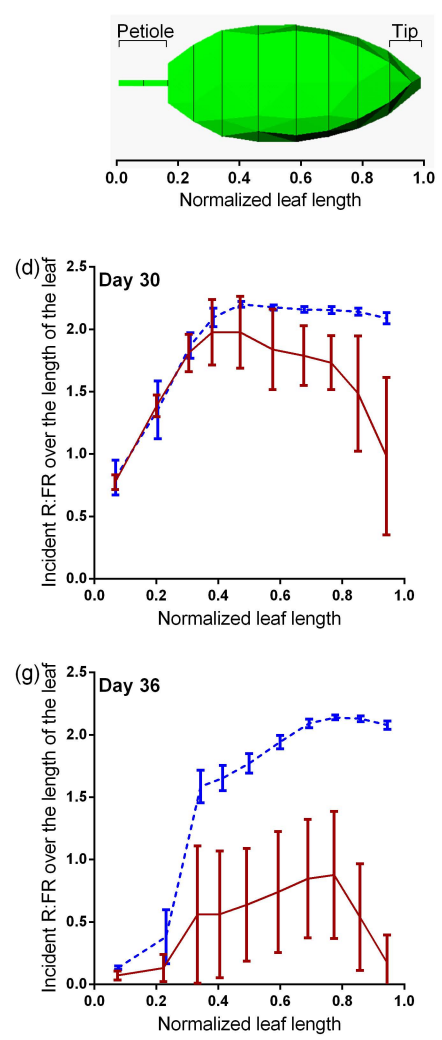
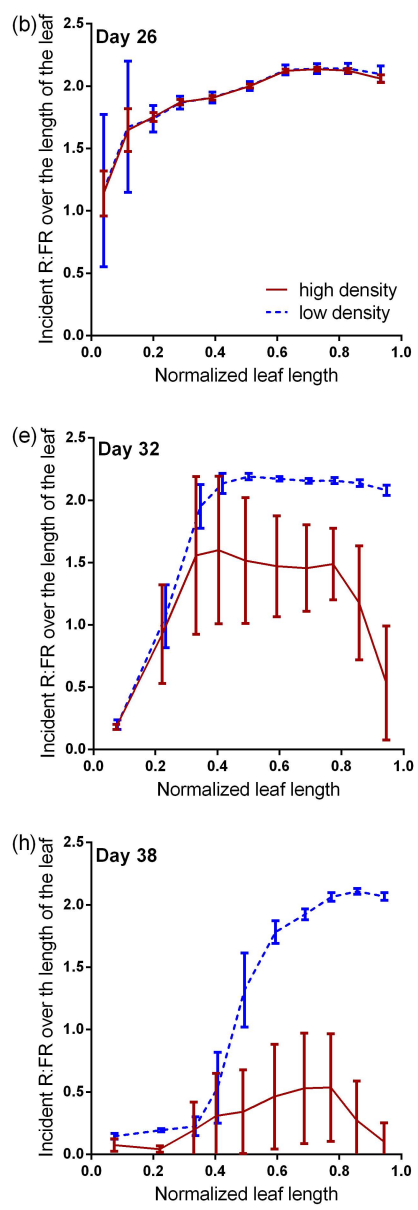
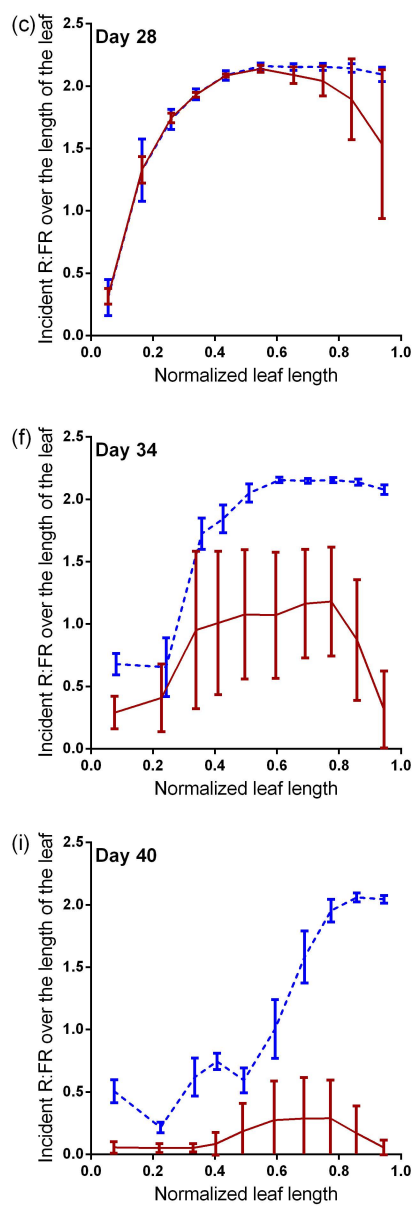

Figure 4.6 Incident R:FR at various sections of the leaf at different moments during the development of a canopy with low and high population density (Scenario 1). (a) Representation of a leaf showing the ten fragments relative to the normalized leaf length. Indicated the sections that represent the petiole and the lamina tip used as R:FR-detection organs in model simulations. (b-i) R:FR at different section over the length of the leaf at day $26,28,30,32,34,36,38$ and 40 in canopies at a low (100 plants $\mathrm{m}^{-2}$, blue) or high (2500 plants $\mathrm{m}^{-2}$, red) population density.

et al., 2013 for exceptions) focus on uniform whole-plant R:FR treatments. Here we studied how localized low R:FR exposures can elicit pronounced different shade avoidance responses that are adaptive during plant-plant competition for light.

\section{Functionality of localized R:FR detection for different responses}

We found that petiole elongation is induced only when low R:FR exposure occurs on the petiole itself, whereas hyponasty is induced if low R:FR is sensed in the lamina tip of the same leaf (Figure 4.1, 4.2 and 4.3). This low R:FR-induced hyponasty is an example of separation of site of perception and site of response, as hyponasty is a result of cell elongation at the abaxial site of the petiole base (Cox et al., 2004; Polko et al., 2012). As IAA 
at the tip induced hyponasty and auxin transport blockers inhibited hyponasty (Figure 4.4), we confirmed the role of auxin in SAS and as signal transporter. However, whether differences between auxin concentrations or auxin sensitivity underlie the difference between abaxial cell elongation and whole petiole elongation is still to be elucidated.

FSP modelling of Arabidopsis rosettes showed that sensing R:FR at the lamina tip indeed is the most effective way to induce hyponasty in response to the proximity of neighbour plants (Figure 4.7). The lamina tip is the first tissue to approach a neighbour plant and is thus the first to be exposed to neighbour-induced reductions in R:FR in stands of rosette-forming plants such as Arabidopsis (Figure 4.5 and 4.6). The R:FR at the level of the petiole was low even in the absence of neighbours, due to self-shading by overlaying younger leaves, and using this to induce hyponasty would be maladaptive. However, using the R:FR perception at the petiole to induce petiole elongation would be adaptive since it increased the petiole length more than lamina tip perception did, which is beneficial to capture more light in a dense canopy (illustrated in Chapter 3). Insight regarding tissuespecific induced phenotypic responses could help the search for crop ideotypes (i.e. plant model that results in the highest possible yield within a given environment), specifically in greenhouse horticulture where additional lighting can be applied to change plant phenotypes with the aim of increasing crop yields (e.g. for tomato, de Visser et al., 2014) .

\section{Model assumptions}

Compared to the previously published model version (Chapter 3), minor adjustments have been made. The most important adjustment is excluding the touch induced-hyponasty, which was indicated as crucial phenotypic response to decrease R:FR ratios in a flat Arabidopsis canopy (de Wit et al., 2012). Including this touch-induced hyponasty, however, did not change the conclusion that using R:FR perception at the lamina tip would be adaptive compared to using the R:FR ratio at the petiole for inducing hyponasty (Supporting Information Figure S4.3). Although both plant types timed their upward leaf movement similarly during canopy development, the greater leaf inclination angle of the 'Petiole' plants compared to the 'Tip' plants had negative consequences for light capture (because of the general decrease in light capture with inclined leaves as also illustrated in Chapter 3). 


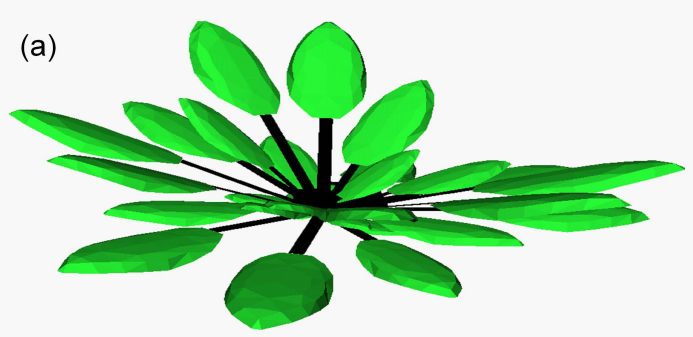

(b)
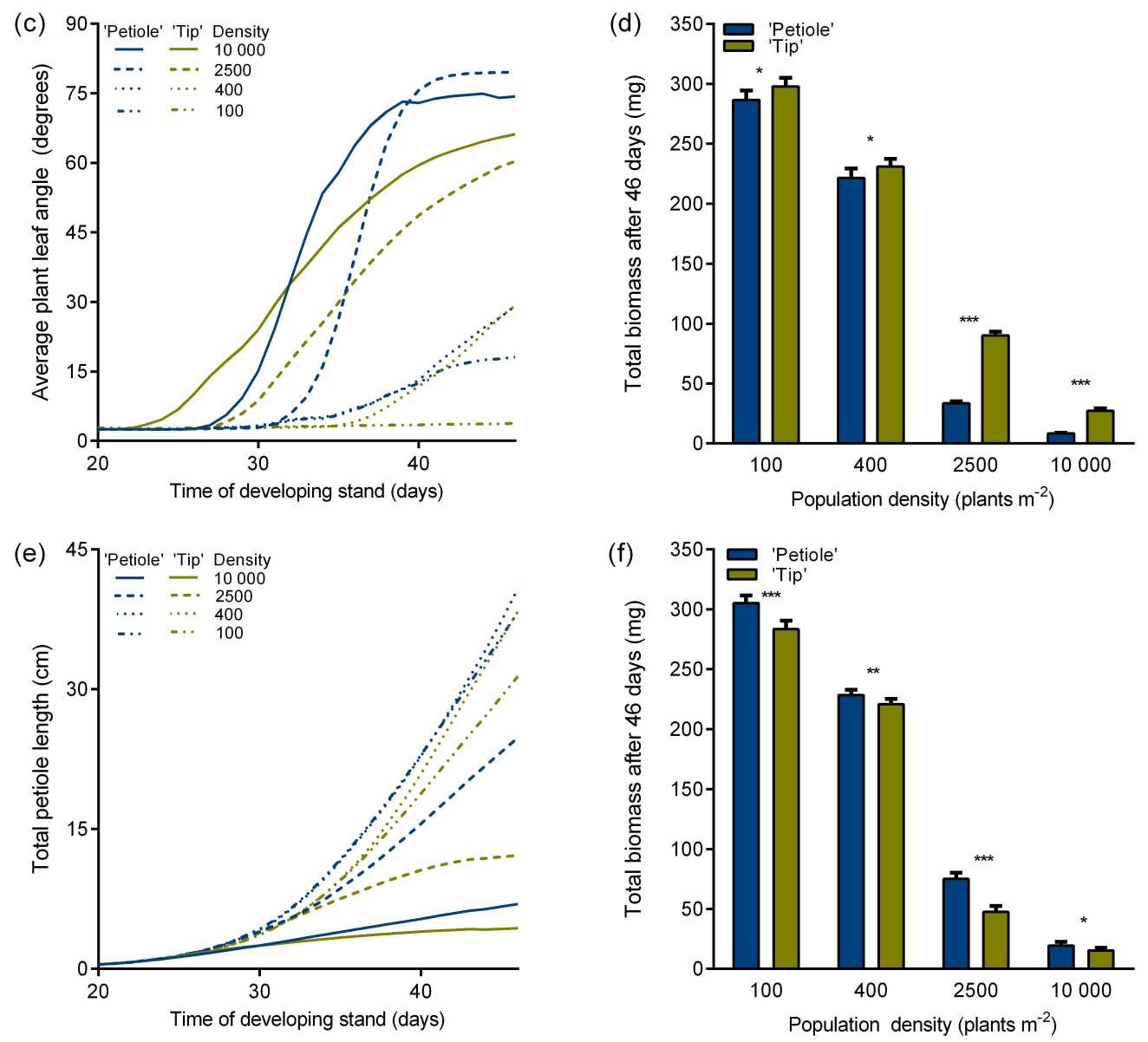

Figure 4.7 Simulated plant leaf angle, petiole length and plant performance of plants growing in different population densities (Scenario 2 and 3). Two plant types were grown in four different $50-50 \%$ mixture canopies at $100,400,2500$ or 10000 plants $\mathrm{m}^{-2}$. 'Petiole' plant types (blue) used the petiole and 'Tip' plant types (yellow) used lamina tip as R:FR-detecting organ to induce hyponasty (Scenario 2) or petiole elongation (Scenario 3). (a, b) Representation of a 'Petiole' (a) and 'Tip' (b) plant after 46 days of growth at a low population density of 100 plants $\mathrm{m}^{-2}$. Petiole and lamina tip RFR-detection tissue coloured in black for 'Petiole' and 'Tip' plants respectively. (c) Mean plant leaf angle during stands development and (d) accumulated biomass after 46 days for plant types using petiole or lamina tip as R:FR-detecting organ for inducing hyponasty, in four mixture stands with different densities. In this scenario both plant types used R:FR at the petiole to induce petiole elongation. (e) Total petiole length during canopy development and (f) accumulated biomass after 46 days for plants using the petiole ('Petiole') or the lamina tip ('Tip') as R:FR-detecting organ for inducing petiole elongation. In this scenario both plant types used the lamina tip to induce hyponasty. Data represent mean \pm SD $(n=10)$. Statistically significant difference indicated by * for $p<0.05,{ }^{*}$ for $p<0.01$ and for $p<0.001$, Student's T-test. 
Brassica plants do not need touch to induce vertical structures within a canopy (i.e. decrease R:FR ratios), and these plants also showed hyponasty upon specific lamina tip low R:FR perception (Figure 4.3). Simulations performed with a Brassica nigra FSP model (J. de Vries, unpublished), showed that using the R:FR light perceived at the lamina tip compared to whole lamina or lamina base would be adaptive in direct competition. This suggests that lamina tip R:FR perception to induce hyponasty is not only beneficial for flat rosette species such as Arabidopsis, but can be adaptive in more vertical canopy structures as it increases the signal reliability for neighbour proximity.

\section{Conclusions}

Low R:FR-induced hyponasty and petiole elongation are clearly a local response: the leaf that senses low R:FR will respond, other leaves do not. However, depending on the type of response and site of cue detection, we have shown that within a single leaf low R:FRdetection and response can be spatially segregated, for which auxin transport is needed. In addition, we showed that the relation between site of cue detection and inducing a specific response determines plant competitiveness. Plants can thus deal with the spatial heterogeneity in their environment at various levels and respond in a highly localized manner.

\section{Acknowledgements}

We thank Jojanneke Voorhoeve for help with the Brassica nigra. This work was funded by the Netherlands Organisation for Scientific Research (NWO), Vidi grant nr 86512.003 to R.P. and ALW grant nr 821.01.014 to N.P.R.A. 


\section{Supporting Information}

(a)

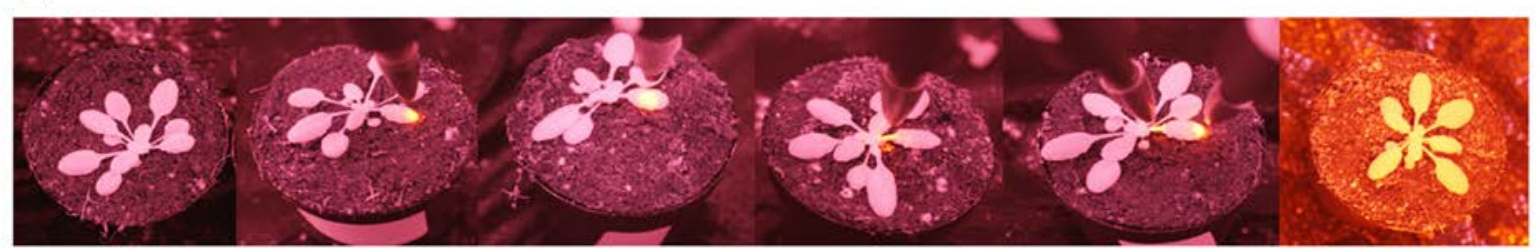

(b)

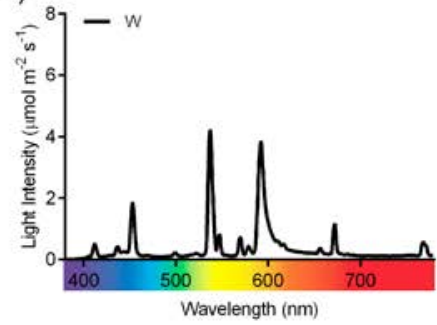

(c)

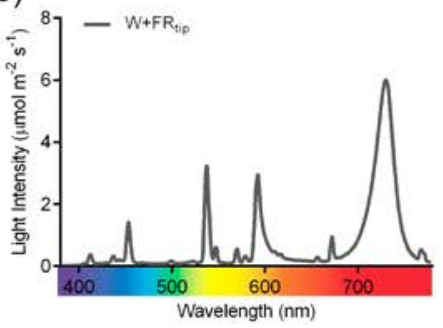

(d)

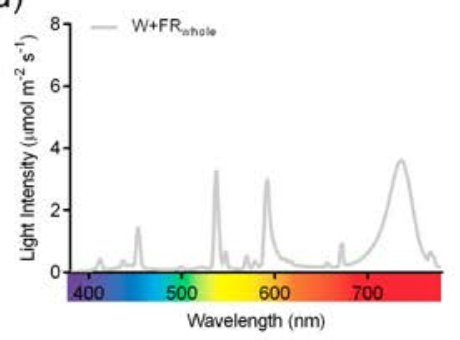

Figure S4.1. Illustrations of localized FR-enrichment and spectrum light composition. (a) Infra-red photographs illustrating plants in the different light treatments; white light (W), white light with supplemental FR through a $3.5 \mathrm{~mm}$ spot (observed as bright yellow spot) on the lamina tip $\left(W+F R_{\text {tip }}\right)$, the middle of the lamina $\left(W+F R_{\text {middle }}\right)$, the petiole

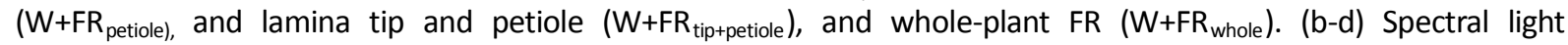
composition of control white light $(W, b)$, white light with supplemental $F R$ spotlight at the lamina tip $\left(W+F R_{\text {tip }}, c\right)$ and white light with supplemental FR at the whole-plant level $\left(W+F R_{\text {whole }}, d\right)$.
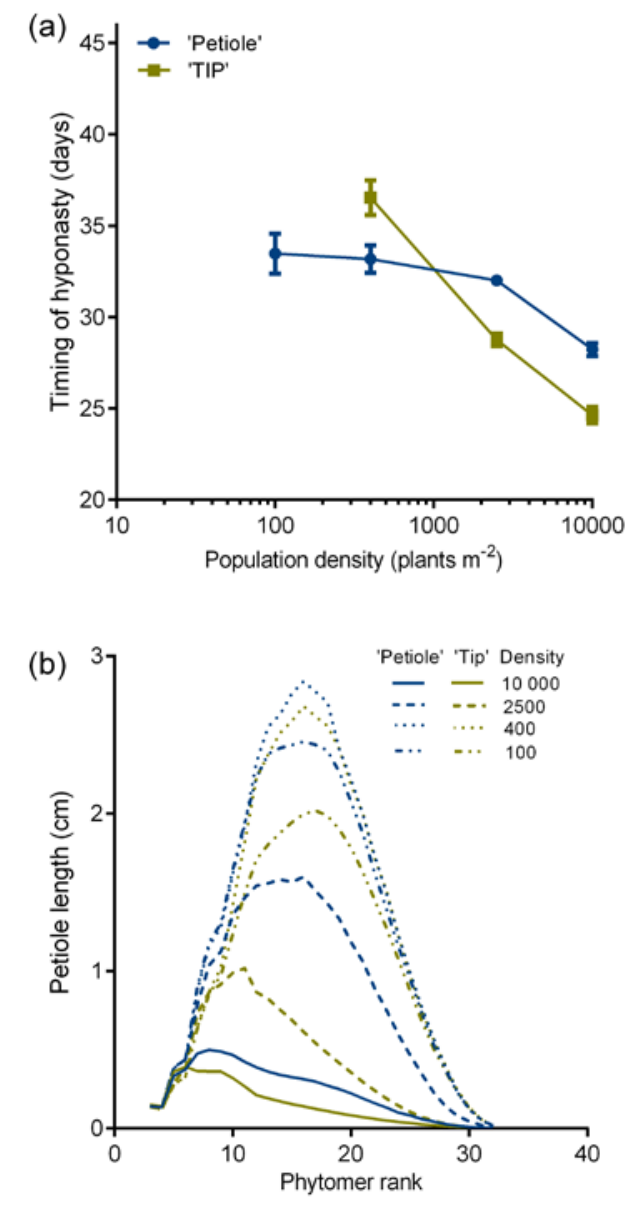

Figure S4.2 Simulated timing of hyponasty and petiole length for two plant types growing in various population densities. (a) Timing of inducing hyponasty of plants using the petiole ('Petiole', blue) or the lamina tip ('Tip', yellow) as R:FR detection tissue when growing in mixtures at various densities. Both plant types used petiole for inducing petiole elongation. (b) Petiole lengths of all organs after 46 days of growth in mixtures of two plant types with different densities (plants $\mathrm{m}^{-2}$ ). The virtual 'Petiole' used the R:FR at the petiole and 'Tip' used the R:FR at the lamina tip to induce petiole elongation. Both plant types used lamina tip for inducing hyponasty. Data represents mean \pm SD (a) or only mean $(n=10)$. 

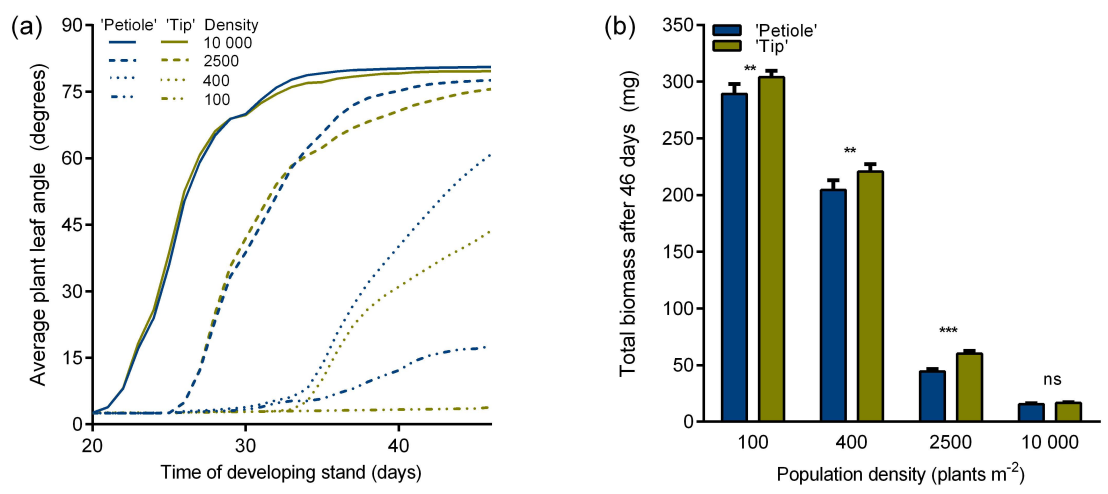

Figure S4.3 Simulated plant leaf angle and plant performance of plants growing in different population densities when also using touch-induced hyponasty as SAS response. Two plant types were grown in four different $50-50 \%$ mixture canopies at $100,400,2500$ or 10000 plants $\mathrm{m}^{-2}$. Both plant types could induce hyponasty based on lamina touch, and in addition the 'Petiole' plant type (blue) used the R:FR at the petiole and the 'Tip' plant type (yellow) used the R:FR at the lamina tip to induce hyponasty. In this simulation both plant types used R:FR at the petiole to induce petiole elongation. (a) Mean plant leaf angle during vegetation development and (b) accumulated biomass after 46 days for plant types using petiole ('Petiole', blue) or lamina tip ('Tip', yellow) as R:FR-detecting organ for inducing hyponasty, in four mixture stands with different densities. Both plant types use R:FR at the petiole to induce petiole elongation. Data represent mean $\pm S D(n=10)$. Statistically significant difference indicated by $*$ for $p<0.05, * *$ for $p<0.01$ and ${ }^{* * *}$ for $p<0.001$, Student's T-test. 
CHAPTER 5

\title{
Variation in plasticity results from selection in different environments
}

\author{
Franca J Bongers ${ }^{1,2}$, Jacob C Douma ${ }^{1}$, Yoh Iwasa ${ }^{3}$, Ronald Pierik ${ }^{2}$, \\ Jochem B Evers ${ }^{1}$, Niels PR Anten ${ }^{1}$ \\ ${ }^{1}$ Centre for Crop Systems Analysis, Wageningen University, Wageningen, the Netherlands \\ ${ }^{2}$ Plant Ecophysiology, Utrecht University, Utrecht, the Netherlands \\ ${ }^{3}$ Theoretical Biology, Kyushu University, Fukuoka, Japan
}




\section{Abstract}

Understanding how natural selection could have acted on the level of phenotypic plasticity in plants is a central question in ecology. Phenotypic plasticity is a vital strategy for a plant to deal with changing environments by inducing favourable phenotypes in different environments. However, phenotypic plasticity is often not represented in current evolutionary and ecologically dynamic population models that are used to understand why and how genotypic composition of plant communities changes in response to environmental change. Here we present a model system that allows for the analysis of selection for variation in phenotypic plasticity, based on evolutionary game theoretical principles. We studied phenotypic plasticity of Arabidopsis thaliana in response to the proximity of neighbour plants as signalled through the decrease in red : far-red (R:FR) ratio using a 3D computational plant model. This model simulates individual plant growth in competition for light, including petiole elongation and lamina growth reduction in response to R:FR signals that are created by the developing canopy itself. Implementing plasticity as a response curve allowed us to describe plasticity based on a single parameter, but still allowing the plants to express different phenotypes in changing environments. We conclude that different plastic response curves are selected for at different population densities. We discuss that the shape of the plastic response curves depends on cue reliability and the negative consequences of plasticity; low cue reliability and high plasticity costs select for low R:FR sensitive response curves. 


\section{Introduction}

In the course of evolution, plants have evolved specific traits (or trait values), which are often environment-specific. When the environmental conditions (i.e. growth conditions) change, this changes the selection on traits and trait values and subsequently the selection of genotypes. This logic forms the basis of a large part of evolutionary and ecological research and has shaped the concept of niche differentiation and its role in driving genotypic diversity and ecosystem functioning (Loreau \& Hector, 2001; Turnbull et al., 2013; Zuppinger-Dingley et al., 2014). However, plants can exhibit multiple phenotypes (i.e. traits) depending on the environment, a phenomenon known as phenotypic plasticity (Bradshaw, 1965; Sultan, 2000; DeWitt \& Scheiner, 2004). Phenotypic plasticity helps a plant to survive in different environments because it allows a plant to change its phenotype matching environmental changes during its life time. Currently evolutionary and ecological dynamic population models are widely used to explain genetic variation and species composition in different environments, and these models are often based on evolutionary game theoretical (EGT) principles (Falster \& Westoby, 2003; Anten \& During, 2011; McNickle \& Dybzinski, 2013; Dybzinski et al., 2015). However, these models do usually not consider phenotypic plasticity and therefore will predict selection for different genotypes in different environments when these environments select for different traits (but see Dybzinski et al., 2013). However, if plasticity would be considered, selection for different traits would not necessarily lead to selection for different genotypes, because a single plastic genotype can express multiple phenotypes.

In general it has been argued that selection will favour those plastic genotypes that induce phenotypic changes such that they have the highest fitness in the whole relevant range of environments (DeWitt et al., 1998; van Kleunen \& Fischer, 2005). The phenotypic change related to an environmental signal can be described by a plastic response curve (see Box 1.1, Chapter 1). The shape of this curve can differ between genotypes, which is referred to as different levels of plasticity. Selection for phenotypic plasticity can result in at least two possible outcomes: i) A single genotype has one response curve that leads to the best phenotypic change to all environmental conditions such that it has the highest fitness across the whole environmental range. ii) The best phenotypic changes to all changes in the 
environment cannot be described by a single response curve, because different environments select for different response curves. The extent to which one or multiple response curves and therefore genotypes are selected for has important implications for plant community diversity, as it determines which genotypes can be selected for (Werner \& Peacor, 2003; Turcotte \& Levine, 2016) and which genotype can become invasive and dominant in a wide range of environments (Richards et al., 2006; Davidson et al., 2011). It can be argued that a single genotype with a specific plastic response curve is more likely to evolve when environmental cues are reliable, that is, when a specific environmental signal always entails the same message regarding the (future) environment. In this case the plants can induce phenotypic changes tailored to all environments, resulting in the highest performance in different environments. If the cue is unreliable, in the sense that its message differs between environments, plants will not always induce phenotypic changes that best fit the environment based on a single response curve. Therefore we hypothesise that if a cue has different messages in different environments, these environments will select for multiple response curves.

To analyse selection on variation in phenotypic plasticity, we focus on plastic responses to neighbour plants. One of the best-known forms of phenotypic plasticity in plants is that plants growing at high density (close proximity to neighbour plants) typically exhibit greater elongation rates of leaf supporting structures (i.e. stem, internodes and/or petioles), less branching or tillering and in species such as Arabidopsis thaliana greater leaf inclination angle (hyponasty) than plants growing at low density (Ballaré et al., 1987, 1994; Dudley \& Schmitt, 1996; Ballaré \& Scopel, 1997; Pierik et al., 2004; de Wit et al., 2012). This set of responses is called the shade avoidance syndrome (SAS; reviewed in Franklin, 2008; Casal, 2013; Ballaré \& Pierik, 2017). SAS is associated with competition for light and results in the placement of leaves in highly lit parts of the canopy before neighbour plants do. One of the primary signals that induces SAS responses is a reduction of the red to far-red ratio of light (R:FR), as plants selectively absorb more red and reflect more far-red light (Ballaré et al., 1987). The reduction in R:FR light perceived by an individual is therefore identified as cue for neighbour proximity (Ballaré et al., 1990); low R:FR ratio when neighbours are close (high density) and high R:FR ratios when neighbours are further away (low density). However, R:FR light conditions are also affected by the vertical component of the canopy structure (Chelle 
et al., 2007; de Wit et al., 2012; Crepy \& Casal, 2015) and by self-shading. This can make the R:FR signal not fully reliable as cue for neighbour proximity.

In addition to R:FR, capture of photosynthetically active radiation for carbon assimilation also depends on presence, size and orientation of leaves and other structures of neighbour plants within the canopy. To analyse selection for plastic responses to neighbour proximity, this interaction between plant phenotype and the light environment needs to be accounted for. However, this is not straightforward because it involves the complex feedback that exists between signal and response: changes in R:FR induce responses at organ-level such as petiole elongation, which in turn causes changes in plant architectural phenotype, affecting light capture for growth. The changed phenotype in turn changes the light environment and associated R:FR conditions, inducing new sets of responses and so on. This complex feedback can be captured in so-called functional-structural plant (FSP) models (reviewed in Vos et al., 2010) that can simulate the interaction between plant growth, its 3D structure and the light distribution within the canopy (Cournède et al., 2008; Evers et al., 2010). Organ-level plasticity in response to local R:FR signals and variation therein can be realistically scaled up to whole-plant performance at vegetation level while taking into account phenotype-environment feedbacks that are created by the vegetation itself (Chapter 3; Bongers et al., 2014; Evers \& Bastiaans, 2016).

In this paper we performed an EGT analysis in which variation in plasticity was taken into consideration explicitly. We did this by using FSP modelling in combination with data on variation in organ-level plasticity to R:FR. We use this approach to analyse to what extent a genotype with a specific plastic response curve could dominate different environments by inducing optimal phenotypes in multiple environments. We focused on petiole elongation and lamina growth reduction of Arabidopsis thaliana as these are important aspects of shade avoidance (Djakovic-Petrovic et al., 2007; de Wit et al., 2012, 2015). To be able to simulate the consequences of the plastic responses for whole-plant performance, we used an FSP model recently developed (Chapter 3). This FSP model simulates Arabidopsis rosette growth based on light absorption for growth and induces phenotypic changes via a plastic response curve that relates relative organ growth to the perception of R:FR. The ability of the model to simulate effects of variation in this plastic response curves for growth, 
phenotype and light competition was tested in Chapter 3. Thus, the FSP model allows us to analyse how selection depends on the consequences of inducing organ-level plasticity in various environments based on the R:FR reliability as cue for neighbour proximity and the cost associated with inducing various phenotypes.

\section{Material and Methods}

\section{Model description}

A functional-structural plant (FSP) model of Arabidopsis rosette growth and development was constructed using the simulation platform GrolMP v1.5 (previously described in Chapter 3). In this model, Arabidopsis rosettes were represented by a collection of leaves composed of petioles and laminas, in a specific arrangement and emerging at a specific time interval. $A$ root compartment was present but only served as a carbon sink. The simulated light source emitted photosynthetic active radiation (PAR), red (R) and far-red (FR) light and in each model time-step these light rays were reflected, transmitted and absorbed by the petioles and laminas according to their wavelength-specific spectral properties. The laminas individually absorbed PAR light, that was converted into growth substrates via photosynthesis, assuming a hyperbolic light response curve (Goudriaan \& van Laar, 1994). The leaves individually grew in time in three dimensions based on the allocated substrates and locally perceived R:FR ratios (more details below in Organ-level growth and plasticity). Organ-level perception of R:FR ratio induced leaf angle increase, petiole elongation and downregulation of lamina growth. Leaf angle increase occurred when the distance between neighbouring leaves was smaller than $2 \mathrm{~mm}$ or when R:FR perception at the lamina was below a threshold of 0.5 (described in detail in Chapter 3). Petiole elongation and lamina growth downregulation were described by a response curve that illustrates the relationship between relative organ growth and R:FR perception. Total accumulated biomass was an emergent property of the model as whole-plant growth and phenotype depended on the level of competition for light that individual plants experienced with neighbouring plants during their growth.

The simulated light source emitted PAR at an intensity of $220 \mu \mathrm{mol} \mathrm{m} \mathrm{m}^{-2}$ and an R:FR ratio of 2.3, representing typical growth chamber conditions. Border effects were 
avoided using the plot replication functionality of GrolMP. Plant growth started based on carbon reserves in the seed, and plant growth was ended 46 days after emergence. Plant orientation within the canopy created variation in light environment and therefore plant growth and performance. For all model details and parameter settings see Chapter 3 . In the simulation model used for this study, small changes were made regarding the interaction with the light environment. (i) Only leaf laminas and not petioles were considered capable of photosynthesis. The latter were regarded to have a support function only, like in other light competition models (see Anten \& Bastiaans, 2016). (ii) The plastic petiole elongation response was based on the R:FR ratio perceived at the petiole itself, instead of the connected lamina (based on new insights obtained in Chapter 4). (iii) Active downregulation of lamina growth was included as response to R:FR perception, and this was based on R:FR perception at the lamina itself. This was to explore how negative consequences of inducing plastic responses would affect selection.

\section{Organ-level growth and plasticity}

Organ growth was primarily simulated by the partitioning of carbon based on the relative sink strength principle (Heuvelink, 1996), assuming a central pool of substrates and consequently non-hierarchical carbon allocation to the growing organs. The relative sink strength of an organ was expressed as a fraction of total plant sink strength, and determined the demand for substrates for each organ in relation to its age. Organ sink strength was defined as its in size in $\mathrm{cm}^{2}$ for laminas and $\mathrm{cm}$ for petioles per unit time (daily time-steps). The potential growth rate was estimated based on the beta growth function (Yin et al., 2003), a bell-shaped function of organ age which is the first derivative of a sigmoid function with three parameters representing the potential size, the growth duration, and the organ age at which the growth rate is maximal. These parameters were estimated independently for lamina and petiole (see Chapter 3). Every time-step the potential growth rate in size was converted into potential growth in carbon substrates using specific leaf area (SLA; $\mathrm{cm}^{2} \mathrm{mg}^{-1}$ ) and specific petiole length (SPL; $\mathrm{cm} \mathrm{mg}^{-1}$ ). SLA was constant with size and age, but SPL decreased with petiole length, based on observation from experiments (Bongers, unpublished).

Petiole elongation and lamina growth downregulation further influences partitioning 
of carbon to the organs. Petiole elongation and downregulation of lamina growth were described by a plastic response curve expressing relative organ growth to R:FR perception; a function based on experimentally obtained petiole elongation data (equation 5.1; data described in Chapter 3):

$$
\mathrm{R}=\left(\frac{\mathrm{R}: \mathrm{FR}}{2.3}\right)^{-a}
$$

Parameter $a$ (dimensionless) determines the curvature of the response curve and is referred to as the 'plasticity coefficient'. The higher the value of $a$ the more sensitive the plant is to R:FR decrease. $\mathrm{R}$ is the relative organ growth factor which is used to induce petiole and lamina plasticity. Petiole elongation was simulated by multiplying the petiole length with R, taking the RFR perceived by the petiole itself as input. This was calculated every model time-step after simulating petiole growth based on carbon allocation through the relative sink strength principle mentioned previously. Consequently, the longer petiole increased its demand (sink strength) for carbon substrates for the next time-step because of the relationship between SPL and petiole length. $\mathrm{R}$ was also used to express lamina growth downregulation upon R:FR perception, modelled by decreasing the carbon demand (sink strength) of the lamina (equation 5.2):

$$
\mathrm{D}=\left(\frac{\mathrm{Dp}}{\mathrm{R}^{\mathrm{n}}}\right)
$$

where $D$ is the actual lamina carbon demand $\left(\mathrm{mg} \mathrm{d}^{-1}\right)$ influences by R:FR perception, $D_{p}$ the potential lamina carbon demand $\left(\mathrm{mg} \mathrm{d}^{-1}\right)$ calculated by the beta growth function, $R$ the response curve based on R:FR perceived by the lamina (calculated with Eq. 5.1), and $n$ a dimensionless coefficient representing the quantification of the consequences of inducing plasticity; i.e., a high value of $\mathrm{n}$ indicates that increased petiole elongation are related to a rather strong reduction in lamina growth (see Model scenarios). The increased carbon demand for a given petiole and the decreased carbon demand for a given lamina has direct consequences for the absolute carbon allocation to all growing organs; decreased carbon allocation to a lamina can be related to increased carbon allocation to a petiole. Plastic responses could only occur during the actual growth phase of the given organ (based on the beta growth function of Yin et al., 2003). In general, we assumed that plastic petiole 
(a)

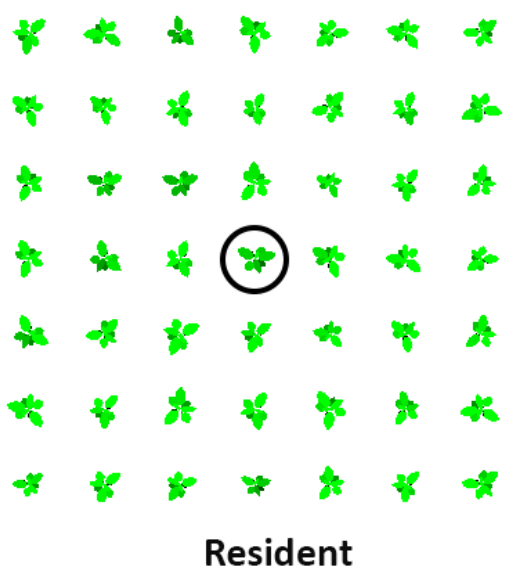

(b)

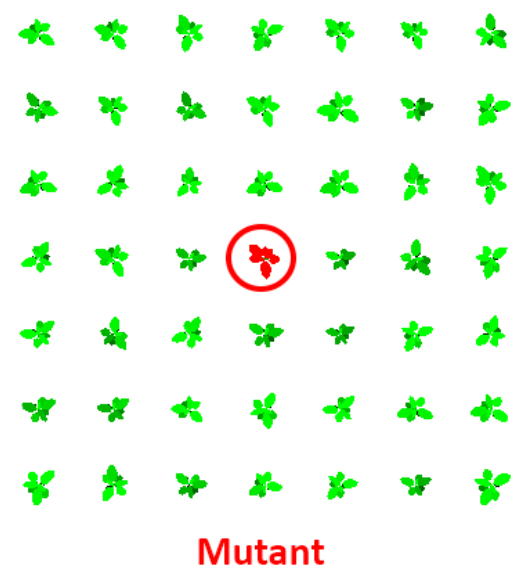

Figure 5.1 Overview of the performed canopy simulations and identified plants used for analysis. The EGT approach assumes that a mutant (genotype with a different strategy) could invade a monoculture population (referred to as resident plants) when the performance of the mutant competing with resident plants is higher than the performance of a resident plant competing with other resident plants. To capture this, we simulated two canopies; one canopy representing a canopy with only resident plants (a) and a canopy representing a single mutant competing with resident plants (b). The different strategy of the mutant compared to the resident was determined by a 0.1 higher plasticity coefficient than the resident plants. For analysis we compared the performance of the middle plants (encircled) of both simulated canopies and these middle plants were referred to as resident or mutant respectively (constructing a resident-mutant pair). Depending on the simulated population density, the number of plants used in the simulations and the distances between these plants that represent the canopy were different, see Table 5.1.

elongation has a positive effect on plant performance as longer petioles could put laminas in a better position of the canopy to increase light capture (illustrated in Chapter 3). Lamina growth reduction was assumed to have negative consequences for plant performance as reduced lamina area captures less light. However, these positive and negative effects depend on population density.

\section{Estimating evolutionary stable strategy}

In general, evolutionary game theoretical (EGT) principles test if a monoculture population (also referred to as 'resident' population) can be invaded by an individual plant that has a different strategy (referred to as 'mutant'). We captured this approach by simulating two canopies (Figure 5.1): one canopy representing only resident plants and a canopy representing a mutant competing with resident plants. To estimate the ability of a mutant to invade a resident population, we compared the performance of the mutant plant growing in the centre of a population of resident plants to a resident plant growing in the centre of a population of resident plants (Figure 5.1). Different strategies are characterized by different 


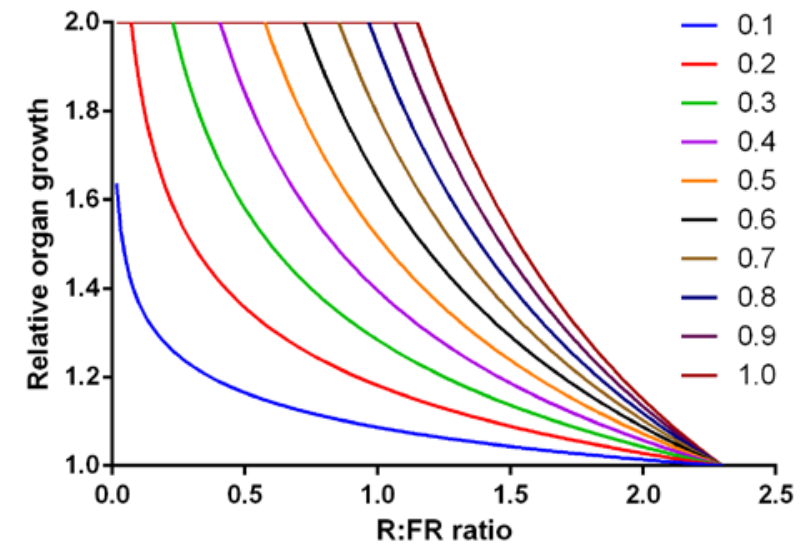

Figure $\mathbf{5 . 2}$ Visualization of different response curves (equation 5.1). Response curves are presented with different values of $a$, also referred to as plasticity coefficient. Different plasticity response curves are created by increasing the value of $a$ from 0 to 1.0 with steps of 0.1 (see legend). Plasticity coefficient of 0 is a horizontal line with value 1 , indicating no plastic response to R:FR light (not visible in the graph). As the plasticity coefficient $a$ increases, response curve are referred to as being more sensitive to R:FR decrease.

values for the plasticity coefficient (parameter $a$ in equation 5.1), representing the variation in the response curves shown in Figure 5.2. Resident populations with different strategies (i.e. plasticity coefficient) were simulated and the plasticity coefficient $a$ of the mutant was always 0.1 higher than those of the resident plants. We chose not to simulate larger (or smaller) differences in plasticity between mutant and resident to minimize computation time, and because we did not search for an absolute ESS value, but for the sensitivity of the ESS for environment and costs related to plasticity (see Model scenarios). Per resident population with a given plasticity coefficient $a$, we conducted 20 replicate simulations of resident against resident and 20 simulations of the mutant against residents, creating 20 resident-mutant pairs. This was done for a range of values of the plasticity coefficient (i.e., 0 - 0.6 in 0.1 steps) and for five population densities. From all resident-mutant pairs, we estimated the relative performance of the mutant by calculating the difference between performance of the mutant and performance of the resident relative to the performance of the resident (equation 5.3):

$$
\mathrm{RPM}=\left(\frac{\mathrm{Pm}-\mathrm{Pr}}{\mathrm{Pr}}\right) * 100
$$

RPM is the relative performance of the mutant (\%), and $\mathrm{Pm}(\mathrm{mg})$ and $\operatorname{Pr}(\mathrm{mg})$ are the performances values of the mutant and resident plants respectively. We used the total accumulated biomass stored in root, laminas and petioles as a measure of plant performance. The rationale is that seasonal seed production (for an annual plant like Arabidopsis this equates to life time reproduction) scales with biomass (Weiner, 1988). 
Generally under light competition this correlation is quite strong (e.g. Ballaré \& Scopel, 1997; Anten et al., 2005; Keuskamp et al., 2010).

To analyse the ability of the mutant to invade a resident population, we used a binary approach based on the 20 resident-mutant pairs: 1 for success (mutant had a higher performance than resident, $\mathrm{Pm}>\mathrm{Pr}$ ) or 0 for failure (mutant had a lower performance than resident, $\mathrm{Pm}<\mathrm{Pr}$ ). We estimated the probability of a mutant to invade a resident population as a function of the plasticity coefficient of the resident population through a logistic regression (generalised linear model with a logit link and binomial error distribution). The plasticity coefficient of the resident population at which the probability of invasion by a mutant would drop below 0.5 was defined the evolutionary stable strategy (ESS; the associated value of the coefficient $a$ is referred to as $\left.a^{*}\right)$. This is based on the assumption that if the probability of a mutant to have a higher performance than the resident is lower than 0.5, the mutant, over multiple generations, will not outcompete the resident population because the resident will more often have a higher performance than the mutant.

\section{Simulations}

First of all, to illustrate the functionality of the 3D growth model, we simulated monocultures of plants that do not show a plastic petiole or lamina response $(a=0)$ at five densities. Next, we explored if the estimated ESS plasticity coefficient a* would be different at different densities (Scenario 1). In addition, we tested if negative consequences of inducing plasticity would alter the estimated ESSs at different densities (Scenarios 2 and 3). Here, we emphasise that we assumed that the benefit of plasticity was created by petiole

Table 5.1 Each scenario was simulated at five population densities, described by the number of plants per square meter. These canopies were constructed by changing the inter-plant distance and the number of plants per canopy to minimize computation time.

\begin{tabular}{|l|c|c|c|}
\hline \multicolumn{1}{|c|}{ Density } & $\begin{array}{c}\text { Population } \\
\text { density } \\
\text { (plants } \mathbf{~}^{-2} \text { ) }\end{array}$ & $\begin{array}{c}\text { Inter-plant } \\
\text { distance } \\
\text { (cm) }\end{array}$ & $\begin{array}{c}\text { Number of } \\
\text { plants in a } \\
\text { canopy }\end{array}$ \\
\hline Extremely High & 6400 & 1.25 & $9 \times 9$ \\
\hline High & 1600 & 2.5 & $7 \times 7$ \\
\hline Medium & 711 & 3.75 & $7 \times 7$ \\
\hline Low & 400 & 5 & $5 \times 5$ \\
\hline Extremely Low & 100 & 10 & $3 \times 3$ \\
\hline
\end{tabular}


elongation while the disadvantage of plasticity was created by the reduced lamina growth (see Organ-level growth and plasticity). Per scenario, five different population densities were simulated by adjusting the distance between plants and altering the number of plants per canopy stand to minimize computation time while ensuring a realistic representation of mutant and resident plant competition (Table 5.1). We altered the negative consequence (disadvantage) of inducing plasticity by adjusting parameter $n$ in equation 5.2 and thereby setting the degree of downregulation of lamina growth upon the perception of R:FR. In Scenario 1, we simulated the downregulation of the potential growth rate of the lamina by setting the value of $\mathrm{n}$ in equation 5.2 to 2 . In Scenario 2 we reduced the negative consequence of plasticity by setting $\mathrm{n}$ to 0 , indicating no active downregulation of lamina growth. In Scenario 3 we assumed a stronger negative consequence of plasticity by setting $n$ to 4 .

\section{Results}

\section{Non-plastic plant growth in monocultures}

We first simulated the growth of plants that did not exhibit plasticity in petiole elongation or lamina growth reduction (plasticity coefficient $a=0$ ). Depending on population density, plants increased their leaf angles at different moments during canopy development. The upward movement of leaves induced by neighbour detection decreased the average R:FR
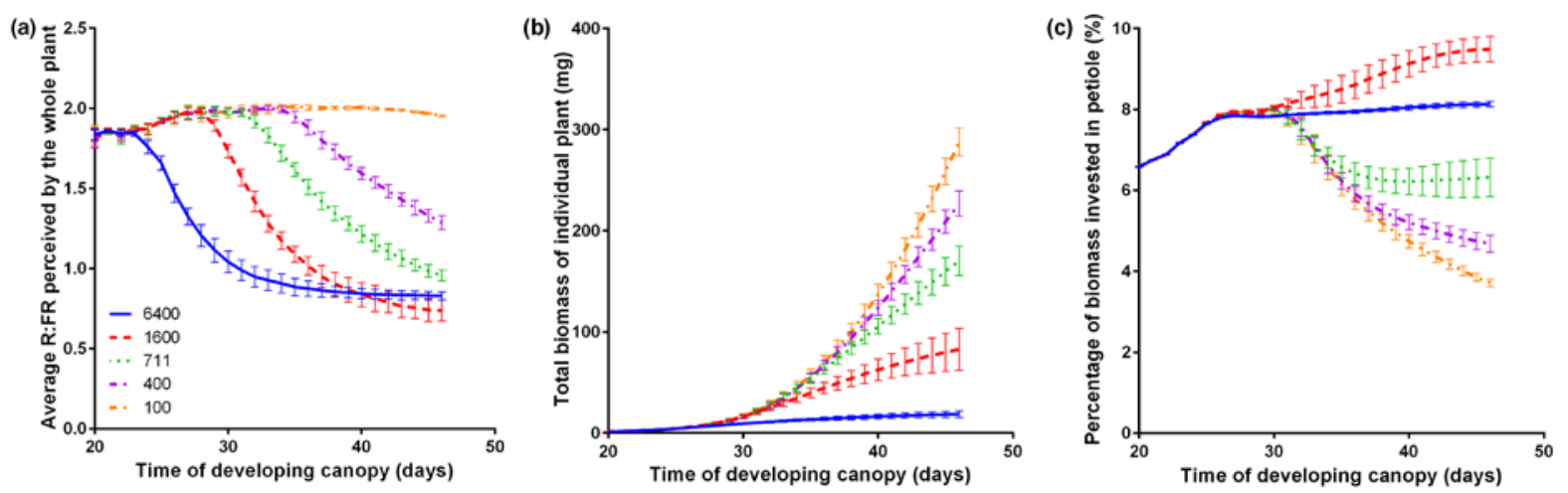

Figure 5.3 Simulated plant characteristics during canopy development of plants types without plasticity growing in monocultures at five population densities. (a) Mean R:FR ratio perceived by the whole plant, (b) total accumulated biomass and (c) percentage of biomass invested in the petiole of an individual plant growing in five densities. Plants grew in monocultures, like resident plants, and did not show plasticity in response to R:FR (plasticity coefficient $a=0$ ). Average perceived R:FR ratio was calculated by dividing the total amount of perceived red light with the total amount of perceived far-red light. Data represent mean $\pm S D(n=20)$. 
ratio perceived by the plant (Figure 5.3a). In addition to the decreasing R:FR ratio, light availability also decreased during canopy development when neighbour plants were present. Therefore, growth based on total accumulated biomass was lower at high than at low densities (Figure 5.3b). Biomass allocation to different organs varied over time and varied with density because the allocation of carbon to growing organs depended on the number of growing organs, their potential growth rate and the light availability in the canopy at any time. Under strong light competition (e.g. at high densities of 1600 and 6400 plants $\mathrm{m}^{-2}$ ) the percentage of biomass allocated to the petiole increased near the end of plant development (Figure 5.3c). However, at weak light competition (low densities), the percentage of biomass allocated to the petiole decreased because petioles required low amounts of carbon compared to the total pool of available carbon and therefore relative more carbon was stored in the root compartment.

The role of plastic responses in carbon allocation patterns and total plant biomass

Plasticity influenced organ biomass allocation and therefore total plant biomass (Scenario 1, Figure 5.4). When plants were growing in a monoculture and thus all plants had the same plasticity coefficient ( $a$ see equation 5.1), plants with higher plasticity coefficients had lower total plant biomass (Figure 5.4a). The plasticity coefficient also affected the biomass allocation to petiole and lamina, independent of density (Figure $5.4 b, c$ ). Because plants with a high
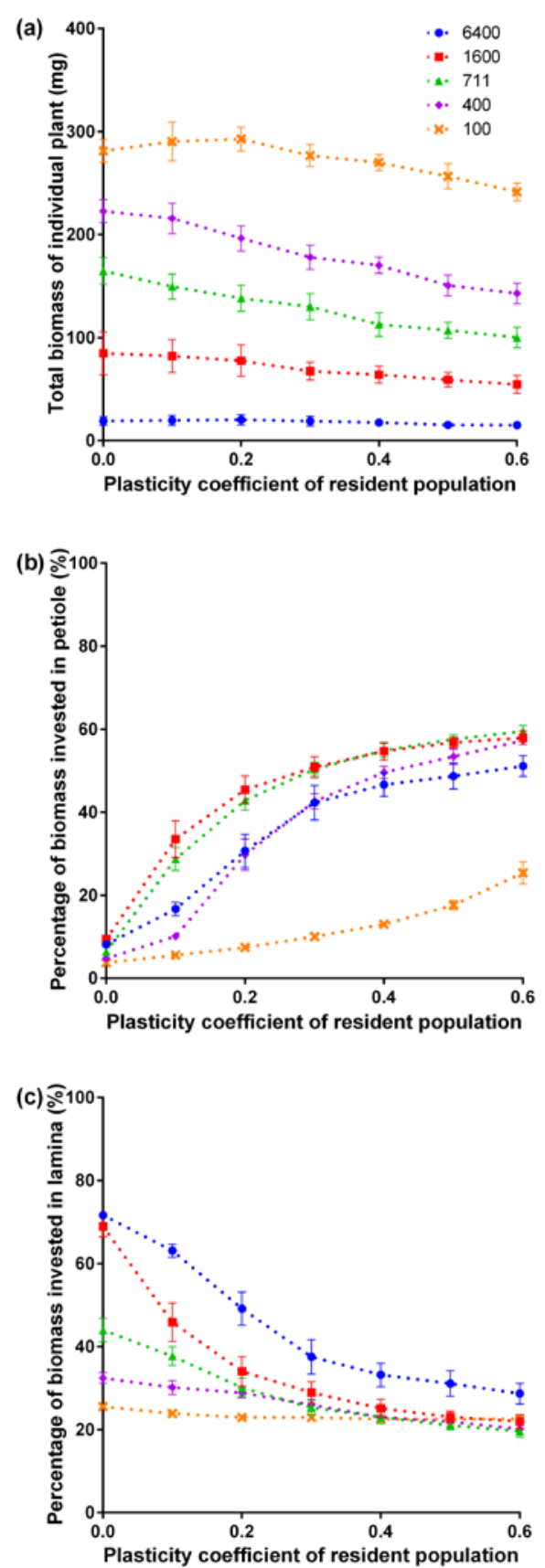

Figure 5.4 Simulated allocated biomass of plant types with different plasticity coefficients growing in monocultures at five population densities (Scenario 1). (a) Total plant biomass, and percentage biomass invested in (b) petiole or (c) lamina of an individual plant at the end of the season, depending on plasticity coefficient and density. Per population density and plasticity coefficient, the canopy consisted of plants with the same plasticity coefficient (also referred to as resident plant). Data represent mean \pm SD $(n=20)$. 
Table 5.2 Mean relative performance values of the mutant (difference between performance of mutant and resident relative to the performance of resident, expressed in percentage; equation 5.3) for each plasticity coefficient $\alpha$ (see equation 5.11 ) of the resident population, at five population densities (plants $\mathrm{m}^{-2}$ ), based on simulations done in Scenario 1. A mutant had an $\alpha$ value that was 0.1 higher than that of the resident population (e.g. in the column 0.1 the $\alpha$ value of residents was 0.1 and that of the mutant 0.2 ). Positive values indicate that the mutant had a higher performance than the resident population, while negative values (red numbers) indicate that the resident population had a higher performance than the mutant.

\begin{tabular}{|l|r|r|r|r|r|r|r|}
\hline & \multicolumn{7}{|c|}{ Plasticity coefficient of resident population } \\
\hline Density & \multicolumn{1}{|c|}{$\mathbf{0}$} & \multicolumn{1}{|c|}{$\mathbf{0 . 1}$} & \multicolumn{1}{c|}{$\mathbf{0 . 2}$} & \multicolumn{1}{c|}{$\mathbf{0 . 3}$} & \multicolumn{1}{c|}{$\mathbf{0 . 4}$} & \multicolumn{1}{c|}{$\mathbf{0 . 5}$} & \multicolumn{1}{c|}{$\mathbf{0 . 6}$} \\
\hline $\mathbf{6 4 0 0}$ & 157.83 & 138.73 & 58.30 & 22.06 & 6.98 & 8.27 & -2.53 \\
\hline $\mathbf{1 6 0 0}$ & 95.02 & 30.81 & 7.10 & 3.02 & -4.09 & -4.11 & -10.94 \\
\hline $\mathbf{7 1 1}$ & 14.87 & 5.90 & 0.68 & -3.86 & -3.36 & -5.12 & -12.05 \\
\hline $\mathbf{4 0 0}$ & 7.01 & 1.01 & -3.40 & -4.54 & -6.87 & -2.06 & -7.20 \\
\hline $\mathbf{1 0 0}$ & 3.90 & 0.62 & -4.67 & -0.68 & -3.81 & -4.76 & -3.12 \\
\hline
\end{tabular}

plasticity coefficient induced plasticity at a relative high R:FR (see Figure 5.2) these plants allocated relatively more biomass to the petioles and less biomass to the laminas compared to plants with a low plasticity coefficient. The absolute amount of biomass allocated to the petiole or lamina was different per density, because of the dynamic allocation pattern of growing plants at various degrees of light competition (as described above).

\section{Stable populations with different plasticity values}

We tested the extent to which the evolutionary stable strategy (ESS; best plastic response curve) depended on population density. When the resident population was not plastic (plasticity coefficient $a=0$ ) or responded at relative low R:FR values ( $a=0.1$ ), we observed that a mutant with a plasticity coefficient 0.1 higher than the resident outcompeted the resident population (relative performance mutant is positive) at every density tested (Table 5.2). The relative performance of the mutant increased with density, from $<5 \%$ in low density to $>130 \%$ in high density. This suggests that there is selection for the shape of a plastic response curve (i.e. plasticity coefficient), regardless of population density, but that the selection pressure is stronger at high than at low density. In addition, with increasing plasticity coefficient of the resident population, the relative performance of the mutant decreased. The plasticity coefficient at which the relative performance is negative is different per density, which suggests that the plasticity coefficient ESS depends on density. 

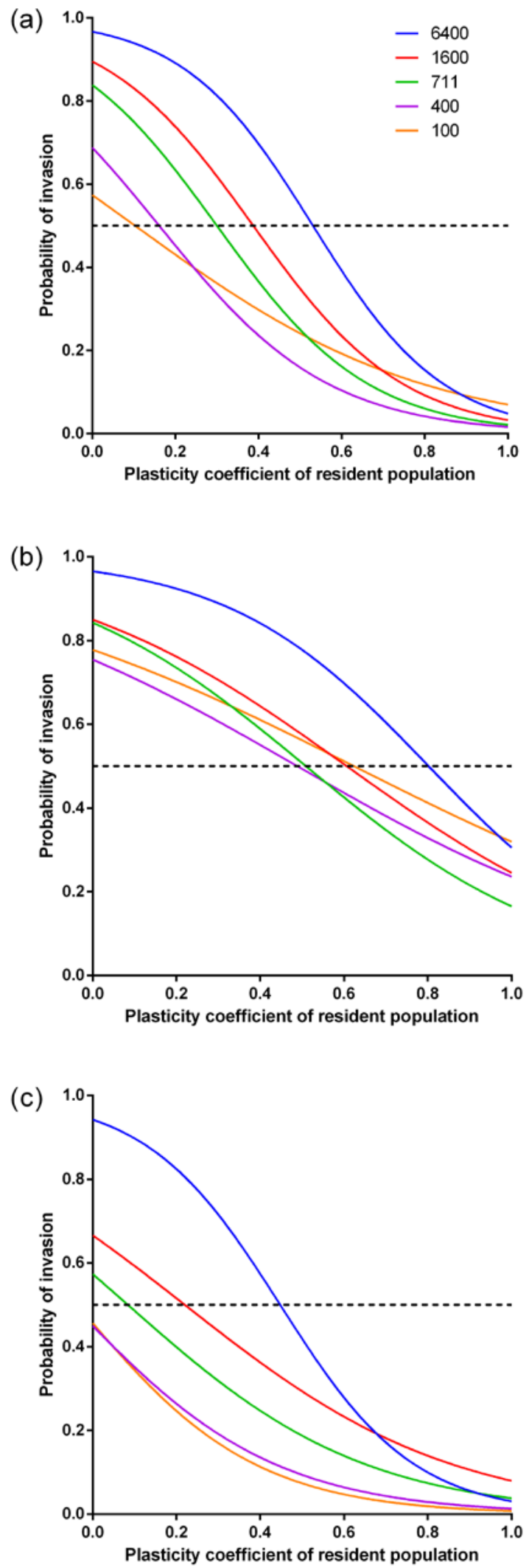

Figure 5.5 Probability of mutant invasion related to plasticity coefficient of the resident population, for five population densities. Horizontal dotted line represent the probability of 0.5 . The point where the probability of invasion is 0.5 was considered the evolutionary stable strategy (ESS; $\left.a^{*}\right)$. (a) Default model setting regarding negative consequences of plasticity (Scenario 1). (b) Minimal negative consequences of plasticity (Scenario 2). (c) High negative consequences of plasticity (Scenario 3). Probabilities were estimated with a logistic regression (GLM, logit link and binomial error distribution) with data from resident-mutant pairs for resident plants with plasticity coefficients of $0,0.1,0.2,0.3,0.4,0.5$ and 0.6 (Scenario 1 \& 3) or additional 0.7, 0.8, 0.9 and 1.0 (Scenario 2). Per resident population with a given plasticity coefficient, $n=20$. See Supplemental Information Table S5.1 for the estimated ESS values per scenario and population density. 
The relationship between the estimated probability for mutant invasion and the plasticity coefficient of the resident population shifted to the right with increasing density, but the slopes of those relationships were not significantly affected by density (Figure 5.5a). Our analysis thus shows that the ESS value $a^{*}$ increased with population density; higher densities selecting for more sensitive responses to decreasing R:FR ratios (Supporting Information Figure S5.1 and Table S1). Our data therefore did not reveal selection for a single genotype with a plastic response curve allowing it to exhibit phenotypes that outcompete any other plant with an alternative plasticity at all population densities, as that would have been reflected in the same $a^{*}$ value at all densities.

Next, we analysed the extent to which the above-mentioned ESS (plastic response curve with coefficient $a^{*}$ ) depended on the negative consequences of plasticity. In two additional scenarios we either decreased (Scenario 2) or increased (Scenario 3) the negative consequences of plasticity by decreasing or increasing the carbon demand for lamina growth upon the perception of R:FR through the plastic response curve. This affected the $a^{*}$ values per density (Figure 5.5 and Supporting Information Figure S5.1 and Table S5.1). With minimal negative consequences (Scenario 2, Figure 5.5b) there was selection towards genotypes with a more sensitive response curve (higher plasticity coefficient), and this effect was stronger at low than at high densities such that the $a^{*}$ values tended to converge. With increased negative consequences of responding to R:FR decrease (Scenario 3, Figure 5.5c) there was selection for genotypes with a lower plasticity coefficient, this effect was again stronger at lower densities. In fact, the $a^{*}$ values at the two lowest densities of 100 and 400 plants $\mathrm{m}^{-2}$ were below 0; indicating that inducing phenotypic change in response to decreasing R:FR at any time was selected against.

\section{Discussion}

In this study, we analysed to which extent a genotype with a specific plastic response curve could dominate different environments by inducing the best phenotype in multiple environments. This was done by expressing phenotypic change at the organ level to an environmental signal (in this case red to far-red light ratios, R:FR) and considering the 
parameter value that defines the shape of the response curve (in this case the plasticity coefficient $a$ ) as a quantitative trait. Using a functional-structural plant (FSP) model allowed us to scale from this organ-level plasticity and variation therein, to whole-plant phenotype and performance at population level, and then to determine the evolutionarily stable response (populations of plants with a plasticity coefficient value that cannot be invaded by plants with a different value) as function of population density. We showed that selection varies with population density, resulting in different evolutionary stable strategies (ESS, i.e. different plastic response curves). This suggests that there is not one single genotype regarding shade avoidance responses; i.e. there is no single genotype with a specific plastic response curve that can grow in different population densities and not be invaded by other genotypes with alternative plastic response curves. In other words, this could mean that as population density typically varies in time and space and if each density selects for a different response, then there is likely to be genetic variation in plasticity. We also showed that when phenotypic changes becomes more costly, selection acted against sensitive responses or even against plasticity at all, depending on the density. Conversely, with plasticity becoming less costly, sensitive responses were selected for and the ESS values for the plastic response curves at different densities slightly converged, suggesting that selection for a single genotype is more likely to occur if negative consequences of plasticity are low.

\section{Unreliable cues select for lower R:FR sensitivity}

It was hypothesised that if an environmental cue is unreliable regarding different environments, selection would probably select for different response curves in different environments. Our results showed that selection for the best response curve indeed changed with population density (Figure 5.5, Supplemental Information Figure S5.1). We suggest that this is partly due to the fact that the reliability of R:FR as cue for neighbour proximity changes with density. In high density stands, R:FR decrease occurs mainly due to neighbour-shading, whereas in low density stands, R:FR reductions are more strongly determined by self-shading. Therefore, the reliability of R:FR as cue for neighbour proximity decreases with lower densities. If the cue is less reliable, the chance of inducing an unfit phenotype increases and then selection will result in the best response curve that minimizes these inductions of unfit phenotypes by adjusting the sensitivity for the R:FR signal. This is 
similar to error management theory that predicts that over evolutionary time, plants will avoid making the most costly wrong phenotype by adjusting cue sensitivity (reviewed in (Johnson et al., 2013; Orrock et al., 2015). Thus, we propose that selection for less sensitive response curves in lower densities is due to the fact that plants in low densities minimized the chance of inducing a wrong phenotype by decreasing their sensitivity to R:FR.

\section{Low plasticity-related costs select for higher R:FR sensitivity}

Changing the negative consequences of plasticity influenced the shapes of the ESS response curves. With higher negative consequences less sensitive response curves were selected for in all densities (Figure 5.5. and Supplemental Information Table S5.1). At the two low densities (100 and 400 plants $\mathrm{m}^{-2}$ ) selection even acted against R:FR sensitivity, resulting in non-plastic genotypes (ESS $a^{*}$ below 0 ). This is again in agreement with predictions of error management theory: if inducing a response will result in high cost, plants that induce less often a response will be selected for, which is illustrated by plants with low sensitive response curve. When the negative consequences were minimal, we predicted larger $a^{*}$ values (compare Figure 5.5b to 5.5a). However, this increase was larger at low than at high density, such that predicted $a^{*}$ values for different densities tended to converge. More similar plastic response curves could in theory mean smaller genetic variation for plasticity and therefore greater likelihood for a single genotype to evolve over a range of population densities.

\section{Modelling choices}

We only simulated plant canopies consisting of plants with similar growth forms in which individuals could always outgrow their competitors. Other studies (e.g. Dudley \& Schmitt, 1995; Weinig, 2000; Donohue et al., 2000) compared low R:FR induced plasticity of ecotypes of the annual dicot Impatiens capensis from open-grasslands versus closed-canopy forests, and found that the ecotypes from the open-grasslands showed greater responsiveness to $R: F R$ decrease than species from closed-canopy forest. In closed-forest canopies, selection should act against inducing plasticity (i.e. increased stem or petiole length) because inducing plasticity could not increase light capture and is therefore maladaptive. Including different growth forms would increase the environment-dependent selection for response curves, due to increased fluctuations of cue reliability. 
Within a vegetation there are more light signals that can indicate shading by neighbour plants. For example the combination of low R:FR and low blue light indicates stronger neighbour-shading, which can be created by increased dense vegetation of plants with similar growth forms or by tall trees as in the closed-canopy forests. In theory this could suggest that species from closed-canopy forests should induce lower SAS responses in the combination of low R:FR and low blue compared to only low R:FR. Where in densegrasslands, the combination of low R:FR and low blue light increasing the cue reliability of the R:FR signal as neighbour proximity and thus should increase the SAS responses compared to only low R:FR (as shown in de Wit et al., 2016b). The reliability of a cue can, thus, be affected by combining different signals together. In addition, the R:FR signal itself can also be more reliable as cue if perception of this signal indicates different environments. For example, perception of low R:FR at the lamina tip is more reliable as cue for neighbourproximity to induce upward leaf movement than R:FR perception at the petiole (see Chapter 4). Taking into consideration that cue reliability can also depend on site of perception or other signals, would make the analysis on selection for one genotype with a specific response curve more complex. However, if this would mean that different environments are better distinguishable, this could eventually lead to one genotype that would dominate various environments.

We assumed that negative consequences of plasticity were equal over different environments and therefore had equal effect on the final cost of inducing a plastic response. However, for example, the vulnerability to mechanical damage or hydraulic limitations for longer petioles can depend on density; in high density canopies, surrounding leaves can give mechanical support or protection against wind, by which plants have lower risk of mechanical failure even if investment in support is low. In low densities, this protection is small (or absent) and therefore long petioles require more investment for the same about off support, which eventually affects final plant performance. If these negative consequences of having longer petioles are indeed larger at low than high density, we would expect an even larger density difference in stable response curves than what we found here. 


\section{Outlook}

Although we simulated a variable light environment, performing the ESS analysis on the densities independently could not take into account the fact that winners in low densities will probably have a great contribution to the genotype performance of the next generations because plants at low density generally receive more light, grow bigger and can thus produce more seeds than winners in high densities. In addition, neighbour-proximity is more heterogeneous in natural vegetation stands than simulated in our equal-spaced canopies, which make the light environment even more variable in time and space. Performing an analysis in which the density between successive generations is variable or the distance between neighbour plants within a whole population is heterogeneous (creating locally different densities), could be a next step towards identifying if one single genotype could dominate in a natural vegetation that is variable for population density.

\section{Conclusion}

Selection for the best plastic response curve depends on the reliability of the cue and negative consequences of inducing plasticity. Using detailed mechanistic 3D plant modelling within an evolutionary game theoretical study is a new step towards analysing how phenotypic plasticity, and the sensitivity for an environmental signal, can affect the composition of plant genotypes within a range of variable environments. Promising next steps could be inclusion of plastic responses to multiple signals instead of one, consideration of potential environmental dependence of negative consequences of inducing phenotypic plasticity and regarding population density to be more variable in time or space.

\section{Acknowledgements}

This work was funded by the Netherlands Organisation for Scientific Research (NWO) ALW grant nr 821.01.014 to N.P.R.A. 


\section{Supporting Information}

Table S5.1 Estimated ESS values $\left(a^{*}\right)$ for the plasticity coefficient of plant populations at various densities and scenarios related to altered negative consequences of inducing plasticity. Negative numbers for the ESS plasticity coefficient indicate that the ESS genotype should not show phenotypic plasticity in response to R:FR.

\begin{tabular}{|l|l|l|l|}
\hline & \multicolumn{3}{|l|}{ Negative consequences of inducing plasticity } \\
\hline $\begin{array}{l}\text { Density } \\
\text { (plants } \mathbf{~}^{-2} \text { ) }\end{array}$ & $\begin{array}{l}\text { Scenario 1 } \\
\text { (default) }\end{array}$ & $\begin{array}{l}\text { Scenario 2 } \\
\text { (low) }\end{array}$ & $\begin{array}{l}\text { Scenario 3 } \\
\text { (high) }\end{array}$ \\
\hline $\mathbf{6 4 0 0}$ & 0.53 & 0.80 & 0.45 \\
\hline $\mathbf{1 6 0 0}$ & 0.39 & 0.61 & 0.22 \\
\hline $\mathbf{7 1 1}$ & 0.30 & 0.51 & 0.08 \\
\hline $\mathbf{4 0 0}$ & 0.16 & 0.49 & -0.05 \\
\hline $\mathbf{1 0 0}$ & 0.10 & 0.62 & -0.04 \\
\hline
\end{tabular}

(a)
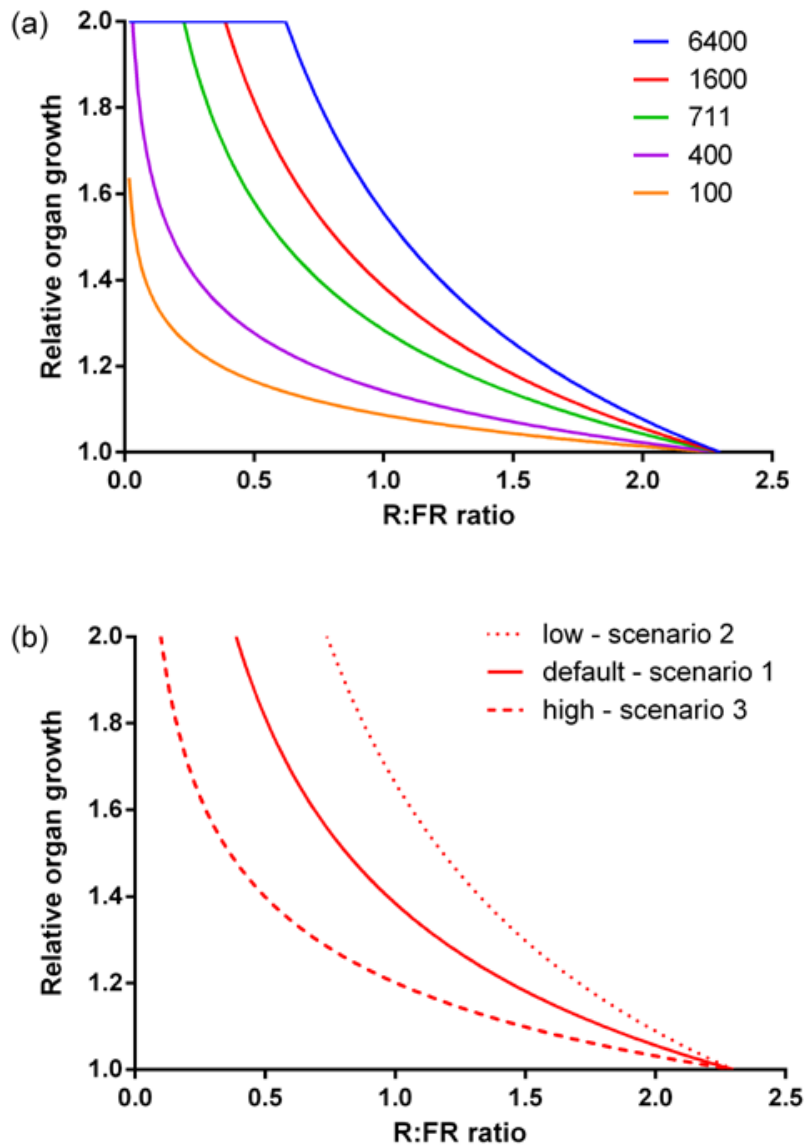

Figure S5.1 Visualization of various ESS response curves. (a) ESS response curves at the five different population densities from Scenario 1, see Table S5.1 for corresponding plasticity coefficient $a^{*}$ values. (b) ESS response curves at a density of 1600 plants $\mathrm{m}^{-}$ ${ }^{2}$ for the three different scenario's in which the negative consequences of inducing plasticity was altered. Response curves are created with equation 5.1. 

CHAPTER 6

General discussion 


\section{Virtual shade sheds light on plant plasticity}

Phenotypic plasticity is the ability of a genotype to express multiple phenotypes across a range of environments (Bradshaw, 1965; Sultan, 2000; DeWitt \& Scheiner, 2004) and it has been established that phenotypic plasticity has ecological benefits in heterogeneous environments. However, there is limited knowledge on how variation in plasticity has consequences for plant competitiveness or how this variation results from natural selection in various environments. In this thesis, I studied how variation in phenotypic plasticity could affect light competition between plants, and in turn, how this variation in phenotypic plasticity could result from selection in different environments. As an exemplary case of phenotypic plasticity, I focused on the petiole elongation and upward leaf movement (hyponasty) responses in the annual rosette plant Arabidopsis thaliana in response to neighbour proximity. These responses are part of the shade avoidance syndrome (SAS), which is an intensively studied form of phenotypic plasticity in plants, and the ratio between red and far-red light (R:FR) is the most important environmental signal that induces these SAS responses. To scale from these plastic responses at the organ-level to whole-plant performance at the vegetation level, I used a functional-structural plant (FSP) model. These FSP models are great tools because they simulate individual plant growth in time and space while explicitly considering the 3D structures of organs that interact with the light environment. This FSP model was used to conduct virtual experiments that simulated various environments in which different plant genotypes competed for light. Thereby I identified that variation in plastic responses affects light competition and also that variation in plastic responses could be the result of selection in different environments.

In this thesis, physiological, ecological and evolutionary processes were linked by combining detailed plant experiments, FSP modelling and evolutionary game theoretical (EGT) principles. In the plant experiments I identified variation in plastic responses, and with the FSP model I quantified how this variation affects plant performance, thereby concluding that even small variation in plasticity can profoundly affect competition for light (Chapter 3 and 4). By simulating light competition between plants in various population densities, considering EGT principles, I concluded that variation in plasticity can be the result of natural selection in various environments (Chapter 5). In this general discussion, I will discuss the 
general conclusions by emphasizing on the dynamic FSP model and the plastic response curve that describes trait responses relative to the R:FR environment. Subsequently, I will discuss additional ideas regarding selection for phenotypic plasticity and the role of the environment and end with a concluding remark.

\section{The role of the functional-structural plant (FSP) model}

Simulating light competition with an FSP model was crucial to study how the level of phenotypic plasticity and variation therein affects light competition between plants. The FSP model explicitly constructed plant phenotypes from individual organs that all interacted with the light environment. This method allowed to scale from organ-level plastic responses to whole-plant performance in different vegetation stands, while regarding the dynamics in the light environment, created by the vegetation itself because only the explicit plant phenotypes (i.e. 3D structures of all organs) interacted with the light. When a plant was growing without direct neighbours (i.e. in a low density of 100 plant $\mathrm{m}^{-2}$ ), all shading (reduction in photosynthetic active radiation (PAR)) and reduction in R:FR that the plant experienced was caused by its own leaves (i.e., self-shading). Conversely, when neighbour plants were present, i.e. in high density of 1600 plant $\mathrm{m}^{-2}$, reductions in PAR and R:FR were mainly determined by the neighbour plants. The light source of the model delivered a constant level of PAR $\left(220 \mu \mathrm{mol} \mathrm{m} \mathrm{s}^{-1}\right)$ and R:FR ratio of 2.3 , which was similar to the light conditions in growth chambers in which the plant experiments were conducted. The organs of the plants changed the light environment (i.e. reduction in R:FR and PAR) due to their specific light optical properties determining organ light absorption, reflection and transmission. That the organ structures absorb light means that the model calculates the partitioning of light over the competing individuals explicitly. This means that this model did not have to make assumptions about how light is partitioned over the individuals that compete for light and have different phenotypes (i.e., trait values). Most other light competition models, do make an assumption about the partitioning of light over individuals related to trait values (Anten, 2005; Pronk et al., 2007; Farrior et al., 2013; van Loon et al., 2014). This method of simulating competition for light does however make other assumptions: e.g. the optical properties of the organs affect the absolute levels of light 

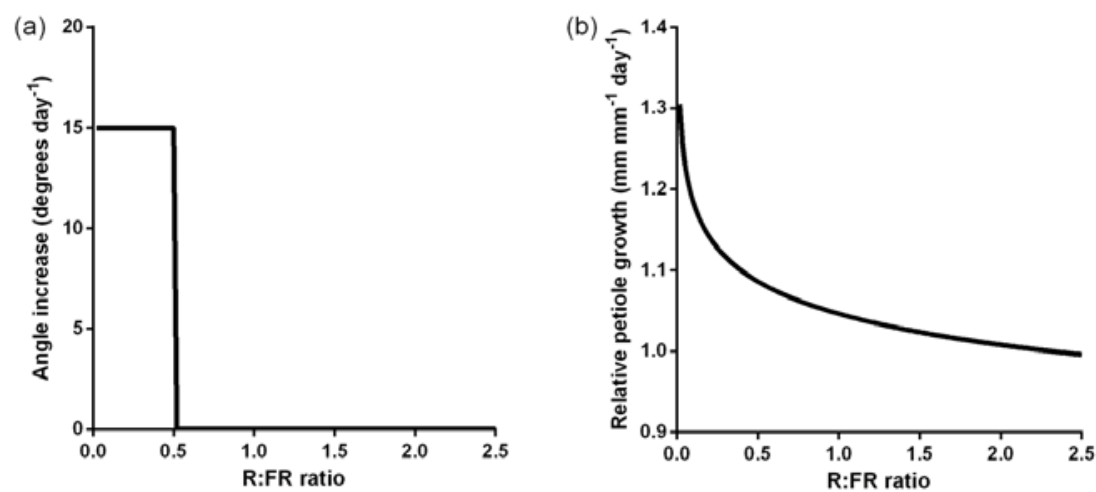

Figure 6.1. Illustration of plastic response curves for hyponasty and petiole elongation. (a) Upward leaf movement was described by a step-wise response curve, exhibiting only two values; 0 degrees angle increase in R:FR higher than 0.5 and 15 degrees angle increase per day in R:FR lower than 0.5. (b) Petiole elongation was described by a gradual response curve, exhibiting various values of petiole elongation related to a range of $\mathrm{R}: \mathrm{FR}$ values described by the function $\mathrm{R}=\left(\frac{\mathrm{R}: \mathrm{FR}}{2.3}\right)^{-a}$, in which $a$ determines the shape of the curve and is referred to as the plasticity coefficient. Here I explain how these response curve induce plastic responses. If a leaf experienced a R:FR value of 0.4 at a given day, the leaf angle would increase with 15 degrees, which entails that if the leaf had already an angle of 15 degrees, the next time-step the leaf angle will be 30 degrees. For the petiole elongation response a R:FR value of 0.4 indicates a relative petiole growth value of 1.1, which entails that as the petiole is $2 \mathrm{~cm}$ long at a given day, the next day this petiole will be $2.2 \mathrm{~cm}$.

absorption and thus shading. However, these assumptions may only affect selection for the absolute levels of plasticity, not the relative effect of selection for different levels in different environments. Simulating competition for light based on growth reduction due to PAR shading in combination with phenotypic changes due to environmental signals is also new compared to previous published FSP models. So far FSP models simulated variation in plant phenotypes based on R:FR responses while organ and plant growth was modelled using empirical rules (Gautier et al., 2000; Evers et al., 2007; Kahlen \& Stützel, 2011). Or conversely, FSP models simulated variation in plant phenotypes based on carbon allocation through light capture but did not include additional R:FR signalling (Cournède et al., 2008; Evers et al., 2010; Evers \& Bastiaans, 2016). Eventually this novel model system analysed selection for different levels of phenotypic plasticity because plasticity was described by a plastic response curve.

\section{The role of the plastic response curve}

In the previous paragraph, I outlined that the FSP model simulated changes in PAR, R:FR and plant phenotype simultaneously in time and space. The model used daily time-steps, which 


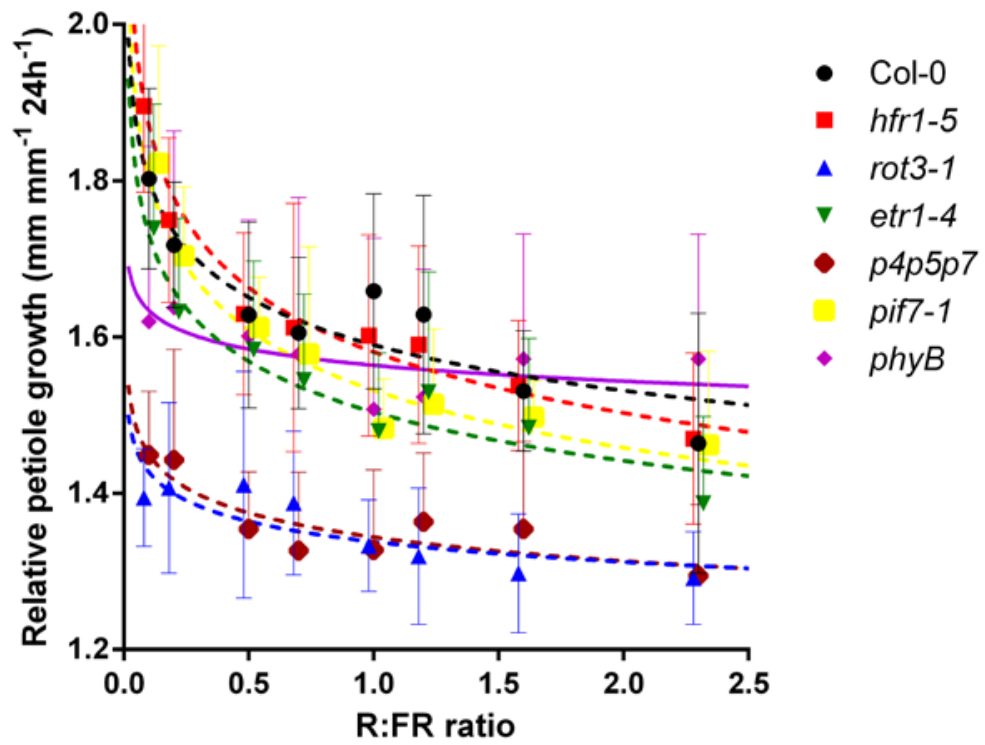

Figure 6.2 Measured petiole elongation response curves for 7 Arabidopsis genotypes. Plants from wild type Arabidopsis (Col-0) and six mutants were subjected to seven values of R:FR and petiole elongation was measured within 24 hours. Data symbols represents mean \pm SD $(n=12)$. Dotted lines represent the fitted curves, based on the function $\mathrm{P}=b^{*} \mathrm{R}: \mathrm{FR}^{-a}$ in which $a$ determines the shape and $b$ illustrates the petiole elongation rate at R:FR of 1.0. See Chapter 3 for more details about the experimental setup and the fitted curves. See literature for more information about the Arabidopsis mutants; hfr1-5 (Sessa et al., 2005), rot3-1 (Kim et al., 1998), etr1-4 (Chang et al., 1993), p4p5p7 represents pif4-101pif5-1pif7-1 (de Wit et al., 2015), pif7-1 (Leivar et al., 2008) and phyB (Reed et al., 1993).

means that every day the light environment was simulated, and every day plant growth was calculated based on light absorption and the plastic responses based on R:FR perception. Because of these dynamic changes in plant phenotype, plasticity was described by a plastic response that expressed trait change in response to R:FR perception within the daily times step of the model. This means that petiole elongation is expressed as relative petiole growth in $\mathrm{mm} \mathrm{mm}^{-1}$ day $^{-1}$ and leaf movement is expressed as angle increase in degrees day ${ }^{-1}$ (see Figure 6.1). Because R:FR can have continues values, the plastic responses were expressed relative to a continues range of R:FR values, which resulted in a step-wise response for hyponasty and a gradual response curve for petiole elongation (Figure 6.1). These response curves have one specific parameter (referred to as the plasticity coefficient) that determined the shape of the response curve, and variation in plasticity was defined by variation in this plasticity coefficient. Variation in the shape of the response curve for petiole elongation was based on plant experiments conducted with Arabidopsis mutants (i.e. genotypes that lost the function of a specific gene) (Figure 6.2). Note that this type of response curve is different 
from reaction norms (Via et al., 1995) often used in ecological studies that describe phenotypic plasticity as static trait differences in relation to a range of environments (Schmitt, 1993; Donohue et al., 2001; Pigliucci \& Schmitt, 2004; Weinig et al., 2006; Dechaine et al., 2007). The combination of quantifying this plastic response curve and explicitly simulating the dynamic light environments based on the presence of other plants (discussed above), enabled me to let a given genotype with one single plasticity value express different phenotypes in different environments, as illustrated in Figure 6.3. The description of phenotypic plasticity as a plastic response curve with a single parameter value was important because this way I could focus on selection for the shape of the plastic response curve that is responsible for the absolute phenotypic values expressed in different environments. Although there are other models that regard the ability of plants to express phenotypic plasticity (Dybzinski et al., 2011, 2013; Farrior et al., 2013), these models focus on the absolute trait values expressed, not on the response that is responsible for that specific trait value, which is the focus in this thesis.

\section{Selection for variation in plasticity}

Combining the measured plastic response curves and the FSP model allowed me to scale from plasticity at the organ-level to whole-plant performance at the vegetation level by which I could study how variation in phenotypic plasticity affected light competition between plants. This in turn allowed me to analyse how variation in phenotypic plasticity could result from selection in different environments. To determine how variation in plasticity affected light competition, 50-50\% checkerboard mixture competition experiments were simulated. In these $50-50 \%$ mixtures two genotypes, with different response curves (Chapter 3) or with different spatial localisation of R:FR perception (Chapter 4), competed for light for 46 days. These simulated competition experiments illustrated that a different shape of the response curve affects plant performance in direct competition because the genotypes had slightly different changes in plant phenotype during vegetation development. A plant competition experiment validated this model simulation by using the same Arabidopsis genotypes that were used for the quantification of the petiole elongation response curves. Additionally, the simulated $50-50 \%$ mixtures illustrated that also variation 
in the R:FR perception-site for inducing petiole elongation or hyponasty has great effect on plant competition for light. This suggests that over generations selection would favour a specific response curve or a specific site of R:FR perception because these plants outcompete plants with another response curve or R:FR perception-site.

To determine if variation in plasticity is the result of selection in different environments, competition experiments based on evolutionary game theoretical (EGT) principles (Chapter 5) were applied, instead of 50-50\% mixtures. This method is accepted as a way to analyse how populations can change due to natural selection (Parker \& Maynard Smith, 1990; Falster \& Westoby, 2003; Anten, 2005; McNickle \& Dybzinski, 2013). These EGT
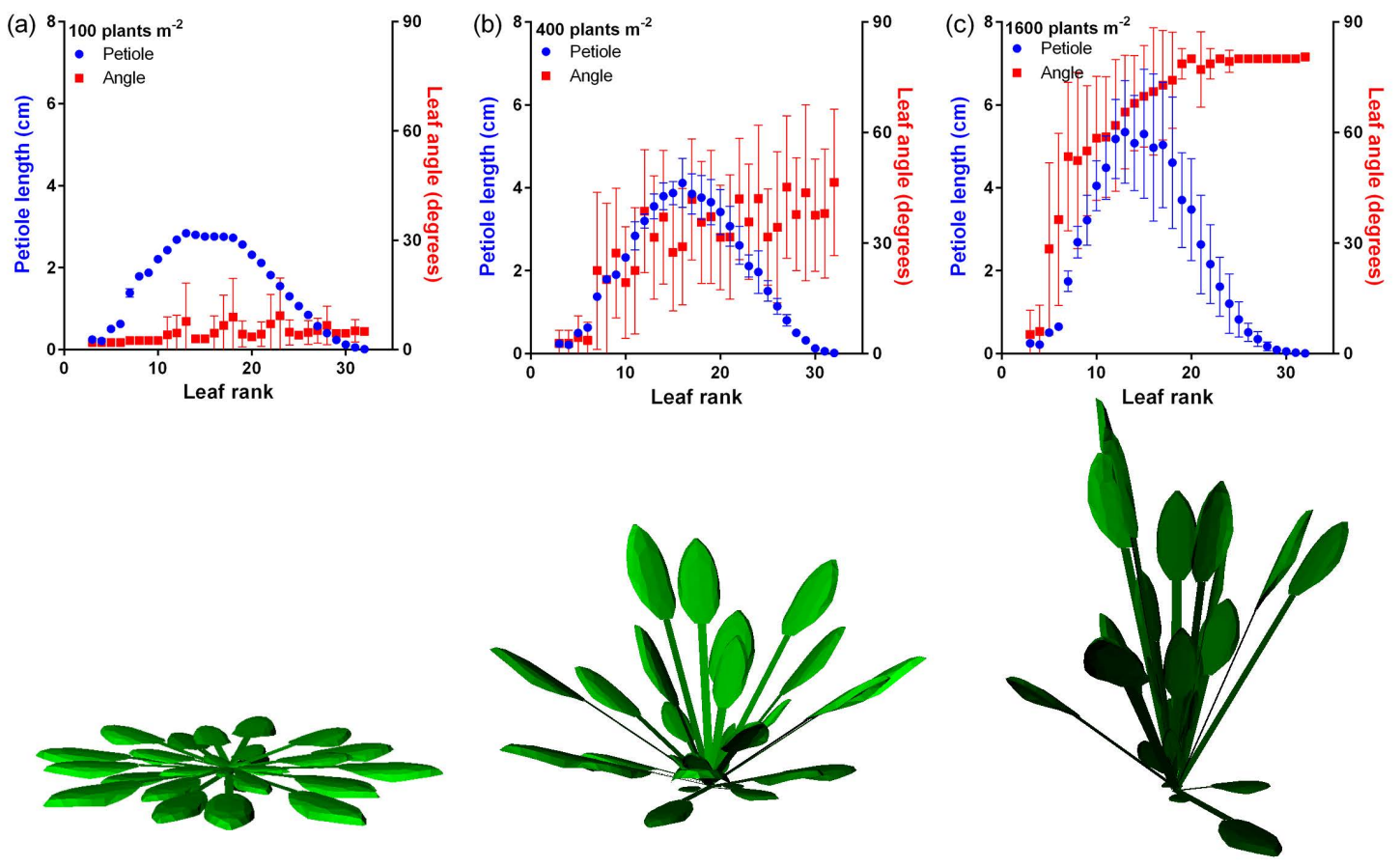

Figure 6.3 Simulated plant phenotypic characteristics after competing for light for 46 days in three different densities. Data is from plants that had a plasticity coefficient $a$ of 0.1 , and competed for 46 days in a monoculture vegetation in which all plants had the same plasticity coefficient. Petiole length (left y-axis) and leaf angle (right y-axis) of all leafs (identified by leaf rank number) are presented. Different panels represent population density of (a) 100, (b) 400, and (c) 1600 plants $\mathrm{m}^{-2}$. Data presents means \pm SD $(n=10)$ and simulated as described in Chapter 5. Per panel also a visualization of a simulated plant after growing 46 days in a monoculture with the corresponding population density. Images of plants are on scale with each other. Intensity of the green colour illustrates the amount of PAR that is absorbed: the darker the colour the lower the amount of PAR absorbed. 
analyses searched for the response curve that would be evolutionary stable: the evolutionary stable strategy (ESS) (Parker \& Maynard Smith, 1990). In theory an ESS is the strategy (i.e. shape of the response curve) that if expressed by all individuals in the whole population could not be invaded by an individual with another strategy (i.e. different response curve). For five population densities I identified five ESSs; per density a different shape of the response curve was selected for. This confirmed the hypothesis that different environments can select for different levels of phenotypic plasticity. The specific shape of the response curve selected for depended on the reliability of the environmental cue, which changed with population density. In low density, a reduction in R:FR signal is almost completely determined by self-shading, whereas in high density the R:FR reduction is mainly caused by neighbour-shading. Additional EGT analyses showed that the shape of the response curve selected for, also greatly depends on the negative consequences of inducing plasticity. Negative consequences of plasticity were simulated by decreasing lamina growth upon the perception of low R:FR. For all the analyses performed, I conclude that variation in plasticity determines selection, and in turn, selection in different environments determines the variation. That selection for the best response in a given environment depends on cue reliability and negative consequences can both be related to the assumption that selection would favour responses that elicit the smallest error (as formulated by error management theory (Johnson et al., 2013; Orrock et al., 2015)). Thus, if cue reliability is low and negative consequences are high, selection would favour responses that are not often induced. In plasticity literature however, evolution of plasticity is mainly analysed by determining the costs and limits of plasticity.

\section{Costs of plasticity}

Costs of plasticity are defined as the lower performance (i.e. lower fitness) of a plastic individual compared to a non-plastic individual while expressing the same phenotype (DeWitt et al., 1998; Auld et al., 2010). On the other hand, limits of plasticity are defined as the development constraint of a plastic individual to express the same phenotype as the non-plastic individual. If costs and limits of plasticity are high, selection would select for nonplastic individuals. However, the definition of costs of plasticity can also be related to 
different levels of plasticity: if costs of plasticity are high, selection would select for less plastic individuals. There are several categories identified within the costs of plasticity; maintenance, production, information acquisition, development instability and genetic costs (DeWitt et al., 1998; Auld et al., 2010). The negative consequences of plasticity mentioned above could be defined as production or developmental-instability costs. Production costs are the costs that plastic individuals have to pay to incur their phenotype compared to nonplastic individuals inducing the same phenotype, and developmental-instability costs are related to the consequences of inducing the imperfect phenotype due to developmental processes. In addition, maintenance cost is defined as cost for maintaining sensory or regulatory systems, and is one of the most intuitive and therefore most studied aspects of plasticity cost. Note that the definitions of costs and limits can be alternative views on the same constraints (reviewed in Auld et al., 2010). Although many studies have been conducted to identify costs, experimental quantification has been difficult (Dorn et al., 2000; Weijschedé et al., 2006; Weinig et al., 2006; Dechaine et al., 2007; Huber et al., 2011). The absence of costs can be explained because natural selection should already have selected for a minimal level of costs, or because plasticity is a by-product of selection for other traits. Although experimental studies have searched for costs, to my knowledge only theoretical models have analysed the effect of costs (especially maintenance and production costs) on the level of plasticity (Ernande \& Dieckmann, 2004; Chevin \& Lande, 2015). However, because these studies described purely theoretical analyses, the shape of the plastic responses and the plasticity costs have no real biological connotation. By using our FSP model that represents plant growth in a more realistic manner, I focussed on the biological meaning of the shape of the plastic responses that can be selected for based on costs related to plasticity. In future modelling studies, maintenance and production cost of SAS plasticity could be simulated by, for example, increasing the maintenance respiration based on the level of plasticity (maintenance cost) and increasing growth respiration related to the level of plasticity expressed (production cost). In addition, determining through plant experiments if there are indeed costs for having sensory machinery or for inducing a phenotypic change in response to signal perception would be interesting, but conceptually and experimentally challenging. 


\section{Different approaches to analyse evolutionary selection}

Analysing what kind of trait will be selected for in a given environment can be approached in different ways. As discussed previously, I used EGT principles to analyse if different population densities would select for different levels of plasticity. However, two strong assumptions had to be made to do so. First of all, I assumed that if a rare genotype would have a higher performance than the dominant genotype, the whole population would adopt the trait of the rare genotype in the next generation. In game theoretical terms this is the replicator rule whereby the winner takes all. I argue that this is quite a strong assumption because a population can consist of multiple genotypes and the composition of the plant population will probably change slowly over evolutionary time based on the relative performance of all these genotypes, not within one generation. Secondly, plant population density was not variable within the vegetation or over generations. At each density, plants were placed in a grid by which the distance between all the plants within the vegetation was equal. Although regular plant placement is common in agricultural systems, it will not occur in natural vegetation stands. Based on this analysis in Chapter 5, I concluded that natural selection will not result in one single genotype with a specific response curve that would be stable against invasion (i.e. dominate the whole population). However, one could argue that if you consider that density is variable in time and/or space, that one single genotype could evolve if, on average and over multiple environments, it has a higher fitness than other genotypes. Based on this idea, I performed two extra simulations (see Box 6.1) and asked the question: is selection for one single response curve possible if density is variable?

I chose the five ESSs that were selected for at the five different densities in Chapter 5 and simulated competition for light while local density was variable within a bigger field (Box 6.1) because the plants were placed randomly in the vegetation (similar to the distribution of weed plants in Evers \& Bastiaans, 2016). In addition, I simulated that genotype abundance would change over generations, letting genotype abundance depend on its performance in the previous generation (see Box 6.1 for more information). The new simulations show that when considering variable local densities within a bigger field, the composition of the whole population could evolve such that one or two genotypes could dominate the population (Figure 6.4). In a relatively low field density (sim400 - Figure 6.4a), one genotype 
outcompeted (dominate) the other genotypes, where as in relatively higher field density (sim1600 - Figure 6.4b) two genotypes co-existed. This new simulation experiment illustrates that a genotype with one single response curve could be selected for if the vegetation density is more variable within the population. Conclusions about selection for a single genotype with a single response curve that could dominate a range of environments thus depends on the vegetation variability and the assumed evolutionary analysis performed. As the two simulations with different field densities resulted in different outcomes, it would be interesting to see what would happen with this evolutionary selection process if density would be variable over generations, because the density of the next generation depends on the absolute seed production (as in Pronk et al., 2007). These additional simulations illustrate nicely that FSP models have great potential to be used in more studies that aim at analysing to what extent competition for resources determines selection for traits. For example, Renten \& Poot, (2014) illustrated that the combination of a FSP model and an evolutionary optimization algorithm could determine how rooting strategies (e.g. root structure) should evolve when resources are distributed heterogeneous in time and space. They showed that the evolution of several root traits predicted by the model simulations was in the same direction as has been observed in plant species adapted to the environments similar to the simulated environments. This confirms the power of combining FSP models and evolutionary theory to provide insight into the relationship between plant traits (also plastic responses) and the environmental conditions.

\section{Physiological processes underlying plasticity}

With physiological plant experiments I identified that the level of petiole elongation increases with a decreasing R:FR ratio (Chapter 3 and Figure 6.2), and that petiole elongation and hyponasty are induced specifically by R:FR perception at the petiole or lamina tip, respectively (Chapter 4). The fact that Arabidopsis mutants express variation in the petiole elongation response curves (Figure 6.2) illustrates the involvement of the genes (and related proteins) in the physiological process underlying the plastic responses. In another experiment with pharmacological treatments, I identified that auxin is involved in inducing hyponasty based on lamina tip R:FR perception (Figure 4.4). Additional experiments with 


\section{Box 6.1 Is selection for one single response curve possible if density is variable?}

The five genotypes selected at the five different densities in Scenario 1 in Chapter 5 (see Supplemental Information Figure S5.1 and Table S5.1) were used for two additional simulations. At the start of each simulation, 16 plants per genotype (i.e. $16 \times$ $5=80$ plants in total) were randomly placed in a field of $2025 \mathrm{~cm}^{2}$ or $484 \mathrm{~cm}^{2}$ representing a field population density of $400(\operatorname{sim} 400)$ and $1600(\operatorname{sim} 1600)$ plants $\mathrm{m}^{-2}$, respectively. Placing plants random in a field created local variable densities, because the distances between neighbour plants was variable. This total of 80 plants per field competed 46 days for light, after which the total accumulated biomass per plant was recorded. After these 46 days, the relative performance per genotype at field level was calculated by dividing the sum of biomass per genotype by the total sum of biomass in the field. In the next generation, a new number of plants per genotype were placed in the field (still total 80 plants) depending on this relative performance per genotype. For example, if the relative performance of genotype 1 was 0.4 then the next generation 32 $(0.4 \times 80=32)$ plants from genotype 1 were placed in the field. This process of placing random new plants per genotype in the field continued over 200 generations. If a genotype had such a low relative performance that it could not place a plant in the field is was outcompeted and could not return to the field. The two simulations ( $\operatorname{sim} 400$ and sim1600) were both simulated 11 times.

(a)

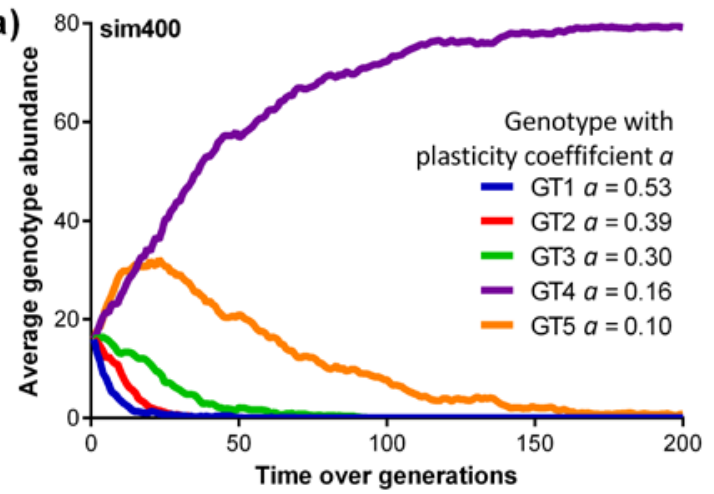

(b)

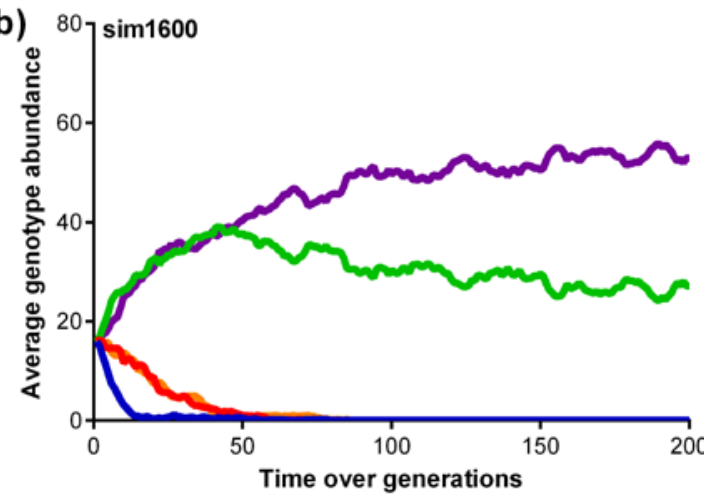

Figure 6.4 Average genotype abundance over $\mathbf{2 0 0}$ generations in a field with population density of $\mathbf{4 0 0}$ (a) and $\mathbf{1 6 0 0}$ (b) plants $\mathrm{m}^{-2}$. The five genotypes represent the genotypes that were selected for in the five densities in Scenario 1, Chapter 5; GT1 was selected for in 6400, GT2 in 1600, GT3 in 711, GT4 in 400 and GT5 in 100 plants $\mathrm{m}^{-2}$. These five genotypes had corresponding different plastic response curves that are represented by their plasticity confident $a$ with value $0.53,0.39,0.30,0.16,0.10$, respectively. The number of plants per genotype in every generation was based on the relative performance of the genotype at field level of the previous generation. Data represents mean $(n=11)$. (a) At 400 plants $\mathrm{m}^{-2}$, GT4 dominated the field after 200 generations. (b) At 1600 plants $\mathrm{m}^{-2}$, GT3 and GT4 co-existed after 200 generations. 
mutants also showed that among others PHYTOCHROME INTERACTING FACTORs (PIFs) are involved in hyponasty induced by R:FR perception at the lamina tip (Pantazopoulou et al., under review). Unfortunately I was limited in measuring more mutants for the petiole elongation response curve. I wanted to use mutants that are altered in hormone production or sensitivity to identify if hormones have different roles in creating the specific shape of the response curve. In other words, are hormones differently related to responses in relative high or low R:FR conditions? Measuring more mutants, or even natural accessions, could than identify if the shape of the response curve could be more variable, meaning that the response curve could be described by different functions with more parameters. However, different functions and more parameters would have made the analysis on how natural selection could have acted on variation in plastic responses more complex.

Identifying that auxin and other factors are involved in SAS related responses is not novel per se (reviewed by Casal, 2012; Ballaré \& Pierik, 2017), neither is identifying the role of these factors in competition for light in a representative vegetation (Schmitt et al., 1995; Pierik et al., 2003; Keuskamp et al., 2010). However, here I analysed selection for the shape of the response curve which would induce the best phenotypes across various dynamic environments. As physiological processes underlie these shapes of the response curves, I indirectly simulated selection for an optimized physiological regulation in response to the environment that is responsible for exhibiting the best phenotypes across a range of environmental conditions. If the aim is to select for different levels of plasticity in cropbreeding programs, knowledge of the genes that are involved is essential, because natural selection acts via the heritability of the genome. In addition, specified knowledge on the site of signal perception for induction of various phenotypic responses can also be crucial for crop-breeding programs. Specifically in greenhouse horticulture that use additional lighting techniques (e.g. de Visser et al., 2014), significantly changing plant phenotypes such that light capture and organ shapes can be optimized to eventually increase crop yields.

\section{Co-regulation of petiole elongation and hyponasty}

Petiole elongation and hyponasty have overlapping physiological mechanisms (e.g. involvement of auxin, reviewed in Casal, 2012; Ballaré \& Pierik, 2017), which would suggest 
co-regulation. In addition, petiole elongation and hyponasty are both the result of cell elongation processes at the petiole base (Cox et al., 2004; Polko et al., 2012, Pantazopoulou et al., under review), further suggesting co-regulation. However, the factors that are involved in both responses can still have different roles in the specific SAS responses. For example, the pif7-1 mutant expresses reduced petiole elongation but a total abolished hyponastic response upon low R:FR perception (compare data from de Wit et al., (2015) and Pantazopoulou et al., under review). This entails that the PIF7 protein is involved in both responses, but has a relative bigger role in hyponasty than in petiole elongation.

From an ecological-evolutionary perspective I would argue that co-regulation of petiole elongation and hyponasty is the result of selection for the best plastic responses in competition for light. For Arabidopsis plants, petiole elongation should only be beneficial in competition for light if it can put the leaves in a higher lit zone of the canopy. In other words, petiole elongation should only occur if the elevated angle is larger than a given threshold. For a Rumex species that escapes flooded conditions, the relatedness of petiole elongation and hyponasty has been illustrated; petiole elongation only occurs when the leaf angle exceeds a certain minimal angle (Cox et al., 2003). For Arabidopsis plants it has, however, been shown that petiole elongation upon low R:FR perception still occurs although the leaf is (deliberately kept) horizontal (de Wit et al., 2012). Nonetheless, because low R:FR conditions in an Arabidopsis stand could only occur through elevated leaf angles (de Wit et al., 2012), I think that selection for petiole elongation in response to R:FR has automatically been selected for while leaf angles are elevated. Simulating plant competition with an FSP model and apply some form of evolutionary algorithm (as is done in Renton \& Poot, 2014) can analyse the best combination of signal sensitivity and responses strength for both hyponasty and petiole elongation responses in competitive settings.

\section{Considering more environmental signals and conditions}

In this thesis, I focussed on the combination of R:FR signalling for plasticity and light absorption for plant growth (i.e. biomass accumulation). However, in nature there are more environmental factors, signals or climatic conditions that affect plant growth. Considering all these aspects is of course not feasible within one study. Nonetheless, while studying 
phenotypic plasticity in response to neighbour proximity there are several other environmental signals to be considered. For example, aboveground, volatiles such as ethylene, or reduced irradiance of blue light also indicate neighbour plants (reviewed in Pierik \& de Wit, 2014) and even induce phenotypic changes that are reminiscent of low R:FRinduced responses (Pierik et al., 2003; Millenaar et al., 2009; Keller et al., 2011; de Wit et al., 2016b). Belowground, neighbour proximity can be indicated by e.g. root exudates or nutrient depletion zones (reviewed in Chen et al., 2012; Pierik et al., 2013). The combination of different signals could increase the cue reliability of neighbour proximity, and thus influence selection for phenotypic responses regarding only R:FR as signal. The combination of low blue light with low R:FR, for example, increases the phenotypic responses (de Wit et al., 2016b). If I would have considered that low blue light occurs during vegetation development and that low blue with low R:FR induce a stronger petiole elongation than solely low R:FR, selection would probably result in the best R:FR response curve that is weaker, and in fact closer to the observed responses (see Figure 6.2 and Supplemental Information Figure S5.1), than was found in the analysis. Considering more cues for neighbour proximity and related phenotypic responses into an FSP model would improve the reality of the model representation of a natural vegetation. However, at the same time it would increase the complexity of the model, the parameter requirement and impede proper interpretation of simulated data.

In addition to the above-mentioned environmental signals that may influence selection processes, there are also other environmental factors that drive plant growth and can thus influence selection. Water and nutrients are important for plant growth next to light, and plants should allocate carbon towards the organ that acquires the most limiting resource (Brouwer, 1963). This implies that plants that compete mostly for light will invest more in aboveground structures like stems or leaves and plants that compete mostly for water or nutrients will invest more in root structures (Dybzinski et al., 2011; Poorter et al., 2012; Kiaer et al., 2013). However, note that above and belowground competition will often, if not always, occur simultaneously, suggesting that plants always have to balance resource investment to shoot and root. In my study, I neglected belowground competition by i) growing plants in individual pots and thus separating the roots in the competition plant experiments and keeping the water and nutrient availability constant and ii) simulating root 
growth solely based on biomass accumulation by light capture and thus not considering water or nutrients for growth. Simulating roots without function and thus assuming that water and nutrients do not affect growth was acceptable in the analysis performed here because R:FR signalling did not affect the relationship between shoot and root growth. However, if carbon allocation to the root would be affected by R:FR signalling, and thus more carbon could be invested in shoot instead of root in low R:FR conditions, the best plastic response curve selected for would have been different. The same holds if water and nutrients would be limited because of low availability or root competition. Including both above and belowground interactions would be a valuable next step in FSP modelling, because both aspects are developed in great detail (see Dunbabin et al., 2013 for a review on root FSP models, and see among others Sarlikioti et al., 2011; Kahlen \& Chen, 2015; Evers \& Bastiaans, 2016 for other aboveground FSP models). However, to understand how competition for both resources is affected by signals and phenotypic responses, it is required to know how signals from aboveground affect belowground structures and the other way around.

\section{Summarizing remark}

As a plant scientist, I want to understand how plants work; from identifying important genes and hormones for trait expression, to the relationship between traits and their role for performance in various environmental conditions. Phenotypic plasticity is a phenomenon that perfectly fits within this learning objective as it requires physiological, ecological and evolutionary knowledge to understand it. I connected these three research domains by combining (physiological) plant experiments, functional-structural plant modelling and applying evolutionary game theoretical principles. Through this combination of tools I could analyse if plants express different levels of phenotypic plasticity (i.e. different plastic response curves) through competition in various environments. As the shape of the plastic response curve depends on the underlying physiological processes, I could indirectly evaluate how the physiological processes that are influenced by environmental conditions and exhibit various trait values across a range of environments, are influenced by natural selection. 
Looking back on the past four years of my PhD study I feel that the power of the approach that underlies this thesis entails the combination of these various tools by which different plant scientific domains are connected. Scaling these different domains will eventually allow for a better understanding of plant-plant interactions and plantenvironment interactions. This knowledge is required to better understand plant function and performance in variable climatic conditions, such as plant growth in agricultural systems. 



\section{References}

\section{A}

Anten NPR. 2002. Evolutionarily Stable Leaf Area Production in Plant Populations. Journal of Theoretical Biology 217: 15-32.

Anten NPR. 2005. Optimal photosynthetic characteristics of individual plants in vegetation stands and implications for species coexistence. Annals of Botany 95: 495-506.

Anten NPR, Bastiaans L. 2016. The use of canopy models to analyse light competition among plants. Canopy Photosynthesis: From Basics to Applications. Springer Netherlands, 379-398.

Anten NPR, During HJ. 2011. Is analysing the nitrogen use at the plant canopy level a matter of choosing the right optimization criterion? Oecologia 167: 293-303.

Anten NPR, Garcia RC, Nagashima H. 2005. Effects of Mechanical Stress and Plant Density on Mechanical Characteristics, Growth , and Lifetime Reproduction of Tabacco Plants. American Naturalist 166: 650-660.

Anten NPR, Schieving F, Werger MJA. 1995. Patterns of light and nitrogen distribution in relation to whole canopy carbon gain in C3 and C4 mono- and dicotyledonous species. Oecologia 101: 504-513.

Anten NPR, Vermeulen PJ. 2016. Tragedies and Crops: Understanding Natural Selection To Improve Cropping Systems. Trends in Ecology \& Evolution 31: 429-439.

Anten NPR, von Wettberg EJ, Pawlowski M, Huber H. 2009. Interactive effects of spectral shading and mechanical stress on the expression and costs of shade avoidance. American Naturalist 173: 241-55.

Auld JR, Agrawal AA, Relyea RA. 2010. Re-evaluating the costs and limits of adaptive phenotypic plasticity. Proceedings of the Royal Society 277: 503-511.

B

Ballaré CL, Pierik R. 2017. The Shade Avoidance Syndrome: Multiple signals and ecological outputs. Plant, Cell and Environment.

Ballaré CL, Sanchez RA, Scopel AL, Casal JJ, Ghersa CM. 1987. Early detection of neighbour plants by phytochrome perception of spectral changes in reflected sunlight. Plant, Cell and Environment 10: 551-557.

Ballaré CL, Scopel AL. 1997. Phytochrome signalling in plant canopies: testing its implications with photoreceptor mutants of Arabidopsis. Functional Ecology 11: 441-450.

Ballaré CL, Scopel AL, Jordan ET, Vierstra RD. 1994. Signaling among neighboring plants and the development of size inequalities in plant populations. Proceedings of the National Academy of Sciences 91: 10094-10098.

Ballaré CL, Scopel AL, Sánchez RA. 1990. Far-red radiation reflected from adjacent leaves: an early signal of competition in plant canopies. Science 247: 329-332.

Bardgett RD, Mommer L, De Vries FT. 2014. Going underground: root traits as drivers of ecosystem processes. Trends in Ecology \& Evolution 29: 692-699.

Barillot R, Escobar-Gutiérrez AJ, Fournier C, Huynh P, Combes D. 2014. Assessing the effects of architectural variations on light partitioning within virtual wheat-pea mixtures. Annals of Botany 114: 725-737.

Berrigan D, Scheiner S. 2004. Modeling the evolution of phenotypic plasticity. Phenotypic plasticity: function and coneptual approaches. New York, NY: Offord University Press., 82-97.

Biedrzycki ML, Jilany TA, Dudley SA, Bais HP. 2010. Root exudates mediate kin recognition in plants. Communicative \& Integrative Biology 3: 28-35.

Bongers FJ, Evers JB, Anten NPR, Pierik R. 2014. From shade avoidance responses to plant performance at vegetation level : using virtual plant modelling as a tool. New Phytologist 204: 268-272.

Boonman A, Anten NPR, Dueck TA, Jordi WJRM, van der Werf A, Voesenek LACJ, Pons TL. 2006. Functional significance of shade-induced leaf senescence in dense canopies: an experimental test using transgenic 
tobacco. The American Naturalist 168: 597-607.

Boonman A, Prinsen E, Voesenek LACJ, Pons TL. 2009. Redundant roles of photoreceptors and cytokinins in regulating photosynthetic acclimation to canopy density. Journal of Experimental Botany 60: 1179-1190.

Bradshaw AD. 1965. Evolutionary Significance of Phenotypic Plasticity in Plants. Advances in Genetics.115-155.

Brouwer R. 1963. Some aspects of the eqiulibrium between overground and underground plant parts. Jaarboek van het Instituur van biologisch and scheikundig onderzoek van landbouwgewassen. Wageningen, 31-39.

C

Casal JJ. 2012. Shade Avoidance. The Arabidopsis Book. American Society of Plant Biologists, e0157.

Casal JJ. 2013. Photoreceptor signaling networks in plant responses to shade. Annual Review of Plant Biology 64: $403-427$.

Casal JJ, Sanchez RA, Deregibus VA. 1986. The effect of plant density on tillering: The involvement of R/FR ratio and the proportion of radiation intercepted per plant. Environmental and Experimental Botany 26: 365-371.

Casal JJ, Smith H. 1988a. Persistent effects of changes in phytochrome status on internode growth in lightgrown mustard: Occurrence, kinetics and locus of perception. Planta 175: 214-220.

Casal JJ, Smith H. 1988b. The loci of perception for phytochrome control of internode growth in light-grown mustard: Promotion by low phytochrome photoequilibria in the internode is enhanced by blue light perceived by the leaves. Planta 176: 277-282.

Chang C, Kwok SF, Bleecker AB, Meyerowitz EM. 1993. Arabidopsis Ethylene-Response Gene ETR1 : Similarity of product to Two-Component Regulators. Science 262: 539-544.

Chelle M. 2005. Phylloclimate or the climate perceived by individual plant organs: What is it? How to model it? What for? New Phytologist 166: 781-790.

Chelle M, Andrieu B. 1999. Radiative models for architectural modeling. Argonomie 19: 225-240.

Chelle M, Evers JB, Combes D, Varlet-Grancher C, Vos J, Andrieu B. 2007. Simulation of the three-dimensional distribution of the red:far-red ratio within crop canopies. New Phytologist 176: 223-234.

Chen BJW, During HJ, Anten NPR. 2012. Detect thy neighbor: Identity recognition at the root level in plants. Plant Science 195: 157-167.

Chevin LM, Lande R. 2015. Evolution of environmental cues for phenotypic plasticity. Evolution 69: $2767-2775$.

Chew YH, Wenden B, Flis A, Mengin V, Taylor J, Davey CL, Tindal C, Thomas H, Ougham HJ, de Reffye P, et al. 2014. Multiscale digital Arabidopsis predicts individual organ and whole-organism growth. Proceedings of the National Academy of Sciences 111: 4127-4136.

Cournède P-H, Mathieu A, Houllier F, Barthélémy D, De Reffye P. 2008. Computing competition for light in the GREENLAB model of plant growth: A contribution to the study of the effects of density on resource acquisition and architectural development. Annals of Botany 101: 1207-1219.

Cox MCH, Benschop JJ, Vreeburg RAM, Wagemaker CAM, Moritz T, Peeters AJM, Voesenek LACJ. 2004. The Roles of Ethylene, Auxin, Abscisic Acid, and Gibberellin in the Hyponastic Growth of Submerged Rumex palustris Petioles. Plant Physiology 136: 2948-2960.

Cox MCH, Millenaar FF, de Jong van Berkel YEM, Peeters AJM, Voesenek LACJ. 2003. Plant Movement . Submergence-Induced Petiole Elongation in Rumex palustris Depends on Hyponastic Growth. Plant Physiology 132: 282-291.

Crepy MA, Casal JJ. 2015. Photoreceptor-mediated kin recognition in plants. New Phytologist 205: 329-338.

D

Davidson AM, Jennions M, Nicotra AB. 2011. Do invasive species show higher phenotypic plasticity than native species and, if so, is it adaptive? A meta-analysis. Ecology Letters 14: 419-431.

Dechaine JM, Johnston JA, Brock MT, Weinig C. 2007. Constraints on the evolution of adaptive plasticity: Costs of plasticity to density are expressed in segregating progenies. New Phytologist 176: 874-882. 
DeJong TM, Da Silva D, Vos J, Escobar-Gutierrez AJ. 2011. Using functional-structural plant models to study, understand and integrate plant development and ecophysiology. Annals of Botany 108: 987-989.

DeWitt TJ, Scheiner SM. 2004. Phenotypic plasticity: Functional and Conceptual Approaches.1-10.

DeWitt TJ, Sih A, Wilson DS. 1998. Costs and limits of phenotypic plasticity. Trends in Ecology \& Evolution 13: 77-81.

Djakovic-Petrovic T, de Wit M, Voesenek LACJ, Pierik R. 2007. DELLA protein function in growth responses to canopy signals. Plant Journal 51: 117-126.

Donohue K, Messiqua D, Pyle EH, Heschel MS, Schmitt J. 2000. Evidence of adaptive divergence in plasticity: density- and site-dependent selection on shade-avoidance responses in Impatiens capensis. Evolution 54: 1956-1968.

Donohue K, Pyle EH, Messiqua D, Heschel MS, Schmitt J. 2001. Adaptive divergence in plasticity in natural populations oflmpatiens capensis and its consequences for performance innovel habitats. Evolution 55: 692702.

Dorn LA, Pyle EH, Schmitt J. 2000. Plasticity to light cues and resources in Arabidopsis thaliana: testing for adaptive value and costs. Evolution 54: 1982-1994.

Dudley SA, Schmitt J. 1995. Genetic differentiation in morphological responses to simulated foliage shade between populations of Impatiens capensis from open and woodland sites. Functional Ecology 9: 655-666.

Dudley SA, Schmitt J. 1996. Testing the Adaptive Plasticity Hypothesis: Density-Dependent Selection on Manipulated Stem Length in Impatiens capensis. American Naturalist 147: 445-465.

Duek PD, Fankhauser C. 2005. bHLH class transcription factors take centre stage in phytochrome signalling. Trends in Plant Science 10: 51-54.

Dunbabin VM, Postma JA, Schnepf A, Pagès L, Javaux M, Wu L, Leitner D, Chen YL, Rengel Z, Diggle AJ. 2013. Modelling root-soil interactions using three-dimensional models of root growth, architecture and function. Plant and Soil 372: 93-124.

Dybzinski R, Farrior CE, Ollinger S, Pacala SW. 2013. Interspecific vs intraspecific patterns in leaf nitrogen of forest trees across nitrogen availability gradients. New Phytologist 200: 112-121.

Dybzinski R, Farrior CE, Pacala SW. 2015. Increased forest carbon storage with increased atmospheric CO2 despite nitrogen limitation: A game-theoretic allocation model for trees in competition for nitrogen and light. Global Change Biology 21: 1182-1196.

Dybzinski R, Farrior C, Wolf A, Reich PB, Pacala SW. 2011. Evolutionarily Stable Strategy Carbon Allocation to Foliage, Wood, and Fine Roots in Trees Competing for Light and Nitrogen: An Analytically Tractable, IndividualBased Model and Quantitative Comparisons to Data. The American Naturalist 177: 153-166.

Ernande B, Dieckmann U. 2004. The evolution of phenotypic plasticity in spatially structured environments: implications of intraspecific competition, plasticity costs and environmental characteristics. Journal of Evolutionary Biology 17: 613-628.

Everhart SE, Askew A, Seymour L, Holb IJ, Scherm H. 2011. Characterization of three-dimensional spatial aggregation and association patterns of brown rot symptoms within intensively mapped sour cherry trees. Annals of Botany 108: 1195-202.

Evers JB. 2016. Simulating Crop Growth and Development Using Functional-Structural Plant Modeling. Canopy Photosynthesis:From Basics to Applications.219-236.

Evers JB, Bastiaans L. 2016. Quantifying the effect of crop spatial arrangement on weed suppression using functional-structural plant modelling. Journal of Plant Research 129: 339-351.

Evers JB, Vos J. 2013. Modeling branching in cereals. Frontiers in Plant Science 4: 399.

Evers JB, Vos J, Chelle M, Andrieu B, Fournier C, Struik PC. 2007. Simulating the effects of localized red:far-red ratio on tillering in spring wheat (Triticum aestivum) using a three-dimensional virtual plant model. New Phytologist 176: 325-336. 
Evers JB, Vos J, Yin X, Romero P, van der Putten PEL, Struik PC. 2010. Simulation of wheat growth and development based on organ-level photosynthesis and assimilate allocation. Journal of Experimental Botany 61: 2203-16.

\section{$\mathbf{F}$}

Falster DS, Westoby M. 2003. Plant height and evolutionary games. Trends in Ecology \& Evolution 18: 337-343.

Farrior CE, Dybzinski R, Levin S a, Pacala SW. 2013. Competition for water and light in closed-canopy forests: a tractable model of carbon allocation with implications for carbon sinks. The American naturalist 181: 314-30.

Fournier C, Andrieu B. 1998. A 3D Architectural and Process-based Model of Maize Development. Annals of Botany 81: 233-250.

Franklin KA. 2008. Shade avoidance. New Phytologist 179: 930-44.

Fraser DP, Hayes S, Franklin KA. 2016. Photoreceptor crosstalk in shade avoidance. Current Opinion in Plant Biology 33: 1-7.

G

Gautier H, Mech R, Prusinkiewicz P, Varlet-Grancher C. 2000. 3D Architectural Modelling of Aerial Photomorphogenesis in White Clover (Trifolium repens L.) using L-systems. Annals of Botany 85: 359-370.

Gommers CMM, Visser EJW, St Onge KR, Voesenek LACJ, Pierik R. 2013. Shade tolerance: when growing tall is not an option. Trends in Plant Science 18: 65-71.

Goudriaan J, van Laar HH. 1994. Modelling potential crop growth processes. Dordrecht: Kluwer Academic Publishers.

Guo Y, Fourcaud T, Jaeger M, Zhang X, Li B. 2011. Plant growth and architectural modelling and its applications. Annals of Botany 107: 723-727.

H

Hemmerling R, Kniemeyer O, Lanwert D, Kurth W, Buck-Sorlin G. 2008. The rule-based language XL and the modelling environment GrolMP illustrated with simulated tree competition. Functional Plant Biology 35: 739750.

Heuvelink E. 1996. Re-interpretation of an Experiment on the Role of Assimilate Transport Resistance in Partitioning in Tomato. Annals of Botany 78: 467-470.

Holmes MG, Smith H. 1977. The function of phytochrome in the natural environment 1-4. Photochemestry and Photobiology 25: 533-557.

Hornitschek P, Kohnen M V, Lorrain S, Rougemont J, Ljung K, López-Vidriero I, Franco-Zorrilla JM, Solano R, Trevisan M, Pradervand S, et al. 2012. Phytochrome interacting factors 4 and 5 control seedling growth in changing light conditions by directly controlling auxin signaling. Plant Journal 71: 699-711.

Hornitschek P, Lorrain S, Zoete V, Michielin O, Fankhauser C. 2009. Inhibition of the shade avoidance response by formation of non-DNA binding bHLH heterodimers. The EMBO journal 28: 3893-902.

Huber H, de Brouwer J, von Wettberg EJ, During HJ, Anten NPR. 2014. More cells, bigger cells or simply reorganization? Alternative mechanisms leading to changed internode architecture under contrasting stress regimes. New Phytologist 201: 193-204.

Huber H, von Wettberg EJ, Aguilera A, Schmitt J. 2011. Testing mechanisms and context dependence of costs of plastic shade avoidance responses in Impatiens capensis (Balsaminaceae). American Journal of Botany 98 : 1602-1612.

Iwasa Y, Cohen D, Leon JA. 1984. Tree Height and Crown Shape, as Results of Competitive Games. Journal of Theoretical Biology 112: 279-297.

Izaguirre MM, Mazza CA, Astigueta MS, Ciarla AM, Ballaré CL. 2013. No time for candy: Passionfruit (Passiflora edulis) plants down-regulate damage-induced extra floral nectar production in response to light signals of competition. Oecologia 173: 213-221. 
Izaguirre MM, Mazza CA, Biondini M, Baldwin IT, Ballaré CL. 2006. Remote sensing of future competitors: impacts on plant defenses. Proceedings of the National Academy of Sciences 103: 7170-4.

Johnson DDP, Blumstein DT, Fowler JH, Haselton MG. 2013. The evolution of error: Error management, cognitive constraints, and adaptive decision-making biases. Trends in Ecology and Evolution 28: 474-481.

K

Kahlen K, Chen T-W. 2015. Predicting Plant Performance Under Simultaneously Changing Environmental Conditions-The Interplay Between Temperature, Light, and Internode Growth. Frontiers in Plant Science 6: 1130 .

Kahlen K, Stützel H. 2011. Modelling photo-modulated internode elongation in growing glasshouse cucumber canopies. New Phytologist 190: 697-708.

Kegge W, Weldegergis BT, Soler R, Vergeer-Van Eijk M, Dicke M, Voesenek LACJ, Pierik R. 2013. Canopy light cues affect emission of constitutive and methyl jasmonate-induced volatile organic compounds in Arabidopsis thaliana. New Phytologist 200: 861-74.

Keller MM, Jaillais Y, Pedmale U V, Moreno JE, Chory J, Ballaré CL. 2011. Cryptochrome 1 and phytochrome B control shade-avoidance responses in Arabidopsis via partially independent hormonal cascades. Plant Journal 67: 195-207.

Keuskamp DH, Pollmann S, Voesenek LACJ, Peeters AJM, Pierik R. 2010. Auxin transport through PIN-FORMED 3 (PIN3) controls shade avoidance and fitness during competition. Proceedings of the National Academy of Sciences 107: 22740-22744.

Kiaer LP, Weisbach AN, Weiner J. 2013. Root and shoot competition: a meta-analysis. Journal of Ecology 101: 1298-1312.

Kim G-T, Tsukaya H, Uchimiya H. 1998. The ROTUNDIFOLIA3 gene of Arabidopsis thaliana encodes a new member of the cytochrome P-450 family that is required for the regulated polar elongation of leaf cells. Genes \& Development 12: 2381-2391.

van Kleunen M, Fischer M. 2005. Constraints on the evolution of adaptive phenotypic plasticity in plants. New Phytologist 166: 49-60.

Kozuka T, Kobayashi J, Horiguchi G, Demura T, Sakakibara H, Tsukaya H, Nagatani A. 2010. Involvement of auxin and brassinosteroid in the regulation of petiole elongation under the shade. Plant Physiology 153: 160818.

de Kroon H, Huber H, Stuefer JF, van Groenendael JM. 2005. A modular concept of phenotypic plasticity in plants. New Phytologist 166: 73-82.

Kunstler G, Falster D, Coomes DA, Hui F, Kooyman, Robert M, Laughlin DC, Poorter L, Vanderwel M, Vieilledent G, Wright SJ, et al. 2016. Plant functional traits have globally consistent effects on competition. Nature 529: 1-15.

L

Leivar P, Monte E. 2014. PIFs: systems integrators in plant development. The Plant Cell 26: 56-78.

Leivar P, Monte E, Al-Sady B, Carle C, Storer A, Alonso JM, Ecker JR, Quail PH. 2008. The Arabidopsis phytochrome-interacting factor PIF7, together with PIF3 and PIF4, regulates responses to prolonged red light by modulating phyB levels. Plant Cell 20: 337-352.

Leivar P, Quail PH. 2011. PIFs: pivotal components in a cellular signaling hub. Trends in Plant Science 16: 19-28.

Li L, Ljung K, Breton G, Schmitz RJ, Pruneda-Paz J, Cowing-Zitron C, Cole BJ, Ivans L, Pedmale U V, Jung H-S, et al. 2012. Linking photoreceptor excitation to changes in plant architecture. Genes \& Development 26: 78590.

van Loon MP, Schieving F, Rietkerk M, Dekker SC, Sterck F, Anten NPR. 2014. How light competition between plants affects their response to climate change. New Phytologist 203: 1253-1365. 
Loreau M, Hector a. 2001. Partitioning selection and complementarity in biodiversity experiments. Nature 412: $72-6$.

M

Maddonni GA, Otegui ME, Andrieu B, Chelle M, Casal JJ. 2002. Maize leaves turn away from neighbors. Plant Physiology 130: 1181-9.

McNickle GG, Dybzinski R. 2013. Game theory and plant ecology. Ecology Letters 16: 545-55.

Millenaar FF, van Zanten M, Cox MCH, Pierik R, Voesenek LACJ, Peeters AJM. 2009. Differential petiole growth in Arabidopsis thaliana: photocontrol and hormonal regulation. New Phytologist 184: 141-52.

M, Saeki T, Schortemeyer M. 2005. On the factor light in plant communities and its importance for matter production. Annals of Botany 95: 549-567.

Morgan DC, Smith H. 1978. The relationship between Phytochrome Photoequilibrium and Development in light grown Chenopodium album L. Planta 193: 187-193.

Mundermann L, Erasmus Y, Lane B, Coen E, Prusinkiewicz P. 2005. Quantitative Modeling of Arabidopsis Development. Plant Physiology 139: 960-968.

$\mathbf{N}$

Nicotra AB, Atkin OK, Bonser SP, Davidson AM, Finnegan EJ, Mathesius U, Poot P, Purugganan MD, Richards CL, Valladares F, et al. 2010. Plant phenotypic plasticity in a changing climate. Trends in Plant Science 15: 68492.

0

Olsen J, Kristensen L, Weiner J, Griepentrog HW. 2005. Increased density and spatial uniformity increase weed suppression by spring wheat. Weed Research 45: 316-321.

Orrock JL, Sih A, Ferrari MCO, Karban R, Preisser EL, Sheriff MJ, Thaler JS. 2015. Error management in plant allocation to herbivore defense. Trends in Ecology and Evolution 30: 441-445.

$\mathbf{P}$

Parker G, Maynard Smith J. 1990. Optimality theory in evolutionary biology. nature 348: 27-33.

Pierik R, Djakovic-Petrovic T, Keuskamp DH, de Wit M, Voesenek LACJ. 2009. Auxin and ethylene regulate elongation responses to neighbor proximity signals independent of gibberellin and della proteins in Arabidopsis. Plant Physiology 149: 1701-12.

Pierik R, Mommer L, Voesenek LACJ. 2013. Molecular mechanisms of plant competition: neighbour detection and response strategies (D Robinson, Ed.). Functional Ecology 27: 841-853.

Pierik R, Visser EJW, De Kroon H, Voesenek LACJ. 2003. Ethylene is required in tobacco to successfully compete with proximate neighbours. Plant, Cell \& Environment 26: 1229-1234.

Pierik R, Voesenek LACJ, de Kroon H, Visser EJW. 2004. Density-Induced Plant Size Reduction and Size Inequalities in Ethylene-Sensing and Ethylene-Insensitive Tobacco. Plant Biology 6: 201-205.

Pierik R, de Wit M. 2014. Shade avoidance: phytochrome signalling and other aboveground neighbour detection cues. Journal of Experimental Botany 65: 2815-2824.

Pigliucci M. 2005. Evolution of phenotypic plasticity: where are we going now? Trends in Ecology \& Evolution 20: $481-6$

Pigliucci M, Pollard H, Cruzan MB. 2003. Comparative studies of evolutionary responses to light environments in Arabidopsis. American Naturalist 161: 68-82.

Pigliucci M, Schmitt J. 2004. Phenotypic plasticity in response to foliar and neutral shade in gibberellin mutants of Arabidopsis thaliana. Evolutionary Ecology Research 6: 243-259.

Polko JK, van Zanten M, van Rooij JA, Marée AFM, Voesenek LACJ, Peeters AJM, Pierik R. 2012. Ethyleneinduced differential petiole growth in Arabidopsis thaliana involves local microtubule reorientation and cell expansion. New Phytologist 193: 339-348. 
Pons TL. 2012. Interaction of temperature and irradiance effects on photosynthetic acclimation in two accessions of Arabidopsis thaliana. Photosynthesis Research 113: 207-19.

Poorter H, Niklas KJ, Reich PB, Oleksyn J, Poot P, Mommer L. 2012. Biomass allocation to leaves, stems and roots: Meta-analyses of interspecific variation and environmental control. New Phytologist 193: 30-50.

Procko C, Crenshaw CM, Ljung K, Noel JP, Chory J. 2014. Cotyledon-Generated Auxin Is Required for ShadeInduced Hypocotyl Growth in Brassica rapa. Plant Physiology 165: 1285-1301.

Pronk TE, Schieving F, Anten NPR, Werger MJA. 2007. Plants that differ in height investment can coexist if they are distributing non-uniformly within an area. Ecological Complexity 4: 182-191.

Prusinkiewicz P, Lindenmayer A. 1990. The Algorithmic Beauty of Plants.

Prusinkiewicz P, Runions A. 2012. Computational models of plant development and form. New Phytologist 193: 549-69.

$\mathbf{R}$

Rankin DJ, Bargum K, Kokko H. 2007. The tragedy of the commons in evolutionary biology. Trends in Ecology \& Evolution 22: 643-651.

Reed JW, Nagpal P, Pooe DS, Furuya M, Chory J. 1993. Mutations in the Gene for the Red / Far-Red Light Receptor Phytochrome B Alter Cell Elongation and Physiological Responses throughout Arabidopsis Development. Plant Cell 5: 147-157.

Renton M, Poot P. 2014. Simulation of the evolution of root water foraging strategies in dry and shallow soils. Annals of botany 114: 763-778.

Richards CL, Bossdorf O, Muth NZ, Gurevitch J, Pigliucci M. 2006. Jack of all trades, master of some? On the role of phenotypic plasticity in plant invasions. Ecology Letters 9: 981-993.

S

Sarlikioti V, de Visser PHB, Buck-Sorlin GH, Marcelis LFM. 2011. How plant architecture affects light absorption and photosynthesis in tomato: towards an ideotype for plant architecture using a functional-structural plant model. Annals of Botany 108: 1065-73.

Sasidharan R, Chinnappa CC, Staal M, Elzenga JTM, Yokoyama R, Nishitani K, Voesenek LACJ, Pierik R. 2010. Light quality-mediated petiole elongation in Arabidopsis during shade avoidance involves cell wall modification by xyloglucan endotransglucosylase/hydrolases. Plant Physiology 154: 978-90.

Scheiner SM. 1993. Genetics and evolution of phenotypic plasticity. Annual Review of Ecology, Evolution, and Systematics 24: 35-68.

Schmitt J. 1993. reaction norms of morphological and life-historu traits to light availability in Imaptiens capensis. Evolution 47: 1654-1668.

Schmitt J, Mccormac AC, Smith H. 1995. A Test of the Adaptive Plasticity Hypothesis Using Transgenic and Mutant Plants Disabled in Phytochrome-Mediated Elongation Responses to Neighbors. American Naturalist 146: 937-953.

Schmitt J, Stinchcombe JR, Heschel MS, Huber H. 2003. The adaptive evolution of plasticity: phytochromemediated shade avoidance responses. Integrative and comparative biology 43: 459-69.

Sessa G, Carabelli M, Sassi M, Ciolfi A, Mittempergher F, Becker J, Morelli G, Ruberti I. 2005. A dynamic balance between gene activation and repression regulates the shade avoidance response in Arabidopsis. Genes \& Development 19: 2811-2815.

Da Silva D, Han L, Faivre R, Costes E. 2014. Influence of the variation of geometrical and topological traits on light interception efficiency of apple trees: Sensitivity analysis and metamodelling for ideotype definition. Annals of Botany 114: 739-752.

Smith H. 2000. Phytochromes and light signal perception by plants-an emerging synthesis. Nature 407: 585-91.

Smith H, Whitelam GC. 1997. The shade avoidance syndrome: multiple responses mediated by multiple phytochromes. Plant, Cell \& Environment 20: 840-844. 
Sultan SE. 2000. Phenotypic plasticity for plant development, function and life history. Trends in Plant Science 5: 537-542.

Sultan SE. 2001. Phenotypic Plasticity for Fitness Components in Polygonum Species of Contrasting Ecological Breadth. Ecology 82: 328-343.

Sultan SE. 2003. Phenotypic plasticity in plants: a case study in ecological development. Evolution \& Development 5: 25-33.

T

Tao Y, Ferrer J-L, Ljung K, Pojer F, Hong F, Long J a, Li L, Moreno JE, Bowman ME, Ivans LJ, et al. 2008. Rapid synthesis of auxin via a new tryptophan-dependent pathway is required for shade avoidance in plants. Cell 133: 164-76.

Turcotte MM, Levine JM. 2016. Phenotypic Plasticity and Species Coexistence. Trends in Ecology \& Evolution 31: 803-813.

Turnbull LA, Levine JM, Loreau M, Hector A. 2013. Coexistence, niches and biodiversity effects on ecosystem functioning. Ecology Letters 16: 116-127.

V

Valladares F, Gianoli E, Gómez JM. 2007. Ecological limits to plant phenotypic plasticity. New Phytologist 176: 749-763.

Valladares F, Sanchez-Gomez D, Zavala MA. 2006. Quantitative estimation of phenotypic plasticity: bridging the gap between the evolutionary concept and its ecological applications. Journal of Ecology 94: 1103-1116.

Vermeulen PJ. 2015. On selection for flowering time plasticity in response to density. New Phytologist 205: 429-4399.

Via S, Gomulkiewicz R, De Jong G, Scheiner SM, Schlichting CD, Van Tienderen PH. 1995. Adaptive phenotypic plasticity: consensus and controversy. Trends in Ecology \& Evolution 10: 212-217.

de Visser PHB, Buck-Sorlin GH, van der Heijden GWAM. 2014. Optimizing illumination in the greenhouse using a 3D model of tomato and a ray tracer. Frontiers in Plant Science 5: 48.

Vos J, Evers JB, Buck-Sorlin GH, Andrieu B, Chelle M, de Visser PHB. 2010. Functional-structural plant modelling: a new versatile tool in crop science. Journal of Experimental Botany 61: 2101-2115.

$\mathbf{W}$

Weijschede J, Berentsen R, Kroon H De, Huber H. 2008. Variation in petiole and internode length affects plant performance in Trifolium repens under opposing selection regimes. Evolutionary Ecology 22: 383-397.

Weijschedé J, Martínková J, de Kroon H, Huber H. 2006. Shade avoidance in Trifolium repens: costs and benefits of plasticity in petiole length and leaf size. New Phytologist 172: 655-666.

Weiner J. 1988. The influence of competition on plant reproduction. In: Doust LJ, ed. Plant Reproductive Ecology: Patterns and Strategies. New York, New York, USA: Oxford Univerisity Press, 228-245.

Weiner J. 1990. Asymmetric competition in plant populations. Trends in Ecology \& Evolution 5: 360-364.

Weinig C. 2000a. Differing selection in alternative competitive environments: shade-avoidance responses and germination timing. Evolution 54: 124-136.

Weinig C. 2000b. Plasticity versus canalization: population differences in the timing of shade-avoidance responses. Evolution 54: 441-451.

Weinig C, Johnston J, German ZM, Demink LM. 2006. Local and global costs of adaptive plasticity to density in Arabidopsis thaliana. American Naturalist 167: 826-836.

Werner EE, Peacor SD. 2003. a Review of Trait-Mediated Indirect Interactions in Ecological Communities. Ecology 84: 1083-1100.

de Wit M, Costa Galvão V, Fankhauser C. 2016a. Light-Mediated Hormonal Regulation of Plant Growth and Development. Annual Review of Plant Biology 67: 513-537. 
de Wit M, Kegge W, Evers JB, Vergeer-van Eijk MH, Gankema P, Voesenek LACJ, Pierik R. 2012. Plant neighbor detection through touching leaf tips precedes phytochrome signals. Proceedings of the National Academy of Sciences 109: 14705-14710.

de Wit M, Keuskamp DH, Bongers FJ, Hornitschek P, Gommers CMM, Reinen E, Martınez-Cerón C, Fankhauser C, Pierik R. 2016b. Integration of Phytochrome and Cryptochrome Signals Determines Plant Growth during Competition for Light. Current Biology 26: 1-7.

de Wit M, Ljung K, Fankhauser C. 2015. Contrasting growth responses in lamina and petiole during neighbor detection depend on differential auxin resde Wit M, Ljung K, Fankhauser C. 2015. Contrasting growth responses in lamina and petiole during neighbor detection depend on differential auxin r. New Phytologist 3: 198-209.

de Wit M, Spoel SH, Sanchez-Perez GF, Gommers CMM, Pieterse CMJ, Voesenek LACJ, Pierik R. 2013. Perception of low red:far-red ratio compromises both salicylic acid- and jasmonic acid-dependent pathogen defences in Arabidopsis. Plant Journal 75: 90-103.

\section{Y}

Yin X, Goudriaan J, Lantinga EA, Vos J, Spiertz HJ. 2003. A Flexible Sigmoid Function of Determinate Growth. Annals of Botany 91: 361-371.

Yu Y, Stomph TJ, Makowski D, van der Werf W. 2015. Temporal niche differentiation increases the land equivalent ratio of annual intercrops: A meta-analysis. Field Crops Research 184: 133-144.

Z

Zhu J, van der Werf W, Anten NPR, Vos J, Evers JB. 2015. The contribution of phenotypic plasticity to complementary light capture in plant mixtures. New Phytologist 207: 1213-1222.

Zuppinger-Dingley D, Schmid B, Petermann JS, Yadav V, De Deyn GB, Flynn DF. 2014. Selection for niche differentiation in plant communities increases biodiversity effects. Nature 515: 108-111. 



\section{English summary}

The functioning and performance of plants is determined by the environment as characterized by abiotic conditions (i.e. climate) and by interactions with other organisms. Plants have evolved specific traits to deal with different environments, and these traits can be fixed per organism or can change in response to changes in the environmental conditions, a phenomenon known as phenotypic plasticity. Phenotypic plasticity is defined as the potential for a genotype to produce multiple phenotypes (i.e. multiple trait values) across a range of environments. Phenotypic plasticity can have great ecological benefits in heterogeneous environments because it can enable plants to induce favourable phenotypes in multiple environments. Although variation in phenotypic plasticity has been observed between plants from different environments, relatively few studies have indicated how this differences in plasticity affect competition or how these differences are the result of competition. In this thesis various analyses are conducted to determine to what extent variation in the level of plasticity affects competition for light and how this variation in level of plasticity could result from selection in different environmental conditions. To perform this analysis, different facets of physiological, ecological and evolutionary principles are combined. Physiological processes are approximated by conducting plant experiments, and with a functional-structural plant (FSP) model the obtained results are scaled up to wholeplant growth at the vegetation-level to analyse the ecological relevance of these physiological processes. Eventually, to regard evolutionary processes, FPS modelling and evolutionary game theoretical (EGT) principles are combined to analyse how natural selection could act on variation in plasticity. In the general introduction, phenotypic plasticity is introduced from different perspectives to outline the role of the abovementioned aspects. Altogether, this thesis focussing on petiole elongation and upward leaf movement (hyponasty) responses in the annual rosette plant Arabidopsis thaliana (Brassicaceae) in response to the proximity of neighbour plants, as signalled through the red : far-red (R:FR) ratio. These responses are part of the shade avoidance syndrome (SAS) that is associated with competition for light by placing leaves in highly lit parts of the canopy before neighbour plants do so. 
In Chapter 2, I present my view on how FSP models are tools that can link shade avoidance responses at the organ level to plant performance at the vegetation level. In which I accentuate that iterative cycles of experimentation and modelling allow for a more holistic understanding of plant-plant and plant-environment interactions.

Chapter 3 consists of three elements. First, the Arabidopsis FSP model is presented that I developed in this thesis and that can quantify plastic responses for light competition. Second, variation in petiole elongation in response to decreasing R:FR is measured in plant experiments and described by a plastic response curve. Third, with experimental and simulated (using the FSP model) competition experiments, the role of this difference in response curve for light competition is analysed. This chapter finally concludes that subtle variation in plasticity affects plant competitiveness, which suggest that selection pressure is likely to have played a role in fine-tuning the sensitive SAS responses found in plants.

In Chapter 4, detailed plant experiments and FSP model simulations are presented related to the R:FR perception site and the induction of petiole elongation and hyponasty. The experiments identified that petiole elongation requires R:FR perception at the petiole and hyponasty requires R:FR perception at the lamina tip. Subsequently, simulations with the FSP model show that inducing petiole elongation and hyponasty based on R:FR perception at the petiole and the lamina tip, respectively, increases plants competitiveness. This is based on the fact that changes in incident R:FR at the petiole and lamina tip depends on population density.

While Chapter 3 and Chapter 4 illustrate that variation in plastic responses affect plant competition for light, Chapter 5 illustrates how selection for variation in the level of plasticity could result from competition in different environments. Here, the Arabidopsis FSP model simulates light competition between genotypes with different plasticity levels, represented by different shapes of the plastic response curve (as measured in Chapter 3). The plastic response curves determine the level of petiole elongation and lamina growth reduction in response to decreasing R:FR. Applying evolutionary game theoretical (EGT) principles onto the FSP simulations reveals that selection at different population densities results in different plastic response curves. Although this analysis concludes that natural selection does not select for a single genotype with a specific plastic response curve that can 
dominate a range of population densities, additional FSP modelling analysis in Chapter 6 challenges this conclusion.

In the general discussion (Chapter 6), the results of Chapter 3-5 are discussed based on the main objective specified in the general introduction (Chapter 1). By discussing explicitly the use of the FSP model in combination with the plastic response curve that represented the physiological plastic response, the essence of this novel approach is emphasised. Subsequently, additional ideas regarding selection for phenotypic plasticity and the role of the environment are discussed. 



\section{Nederlandse samenvatting}

Planten hebben verschillende eigenschappen om om te gaan met de omgeving. De omgeving van de plant bestaat uit klimaat gerelateerde aspecten zoals licht, temperatuur en regen, maar ook de aanwezigheid van andere planten, dieren of mensen. Afhankelijk van de omgeving hebben planten verschillende eigenschappen ontwikkeld, en deze eigenschappen bepalen het fenotype. Ook al zijn veel eigenschappen specifiek voor verschillende plantensoorten, planten kunnen ook hun eigenschappen veranderen als hun omgeving verandert. Het fenomeen dat planten hun fenotype veranderen als de omgeving verandert wordt fenotypische plasticiteit genoemd. Fenotypische plasticiteit kan voordelig zijn omdat planten hierdoor de beste eigenschappen kunnen hebben in verschillende omgevingen. Dat er variatie is tussen individuen in fenotypische plasticiteit is bekend, maar hoe deze variatie het resultaat kan zijn van selectie in verschillende omgevingen of invloed heeft op competitie tussen verschillende planten, is nog onduidelijk. Deze thesis concentreert zich op fenotypische plasticiteit dat te maken heeft met competitie om licht. Licht is cruciaal voor groei, door middel van fotosynthese, en de aanwezigheid van buurplanten die concurreren om het licht heeft daarom veel invloed op hoe planten groeien. In deze thesis zijn verschillende analyses gedaan waarmee is uitgezocht hoe variatie in plastische reacties invloed heeft op competitie voor licht, en hoe deze variatie in plastische reacties het resultaat kan zijn van selectie in verschillende omgevingen, gecreëerd door verschillende dichtheden van buurplanten.

Om deze analyses uit te voeren zijn facetten van planten fysiologie, ecologie en evolutie gecombineerd, waarvoor gebruikt gemaakt is van plant experimenten, een 3dimensionaal computermodel van planten en evolutionaire spel-theoretische principes. In de algemene introductie is fenotypische plasticiteit benaderd aan de hand van deze facetten, en is het voorbeeld van fenotypische plasticiteit in reactie op licht competitie geïntroduceerd. In deze thesis wordt gebruikt gemaakt van fenotypische plasticiteit van de rozet plant Arabidopsis thaliana (zandraket), te weten petioolstrekking en toename van de bladhoek (ook wel hyponastie genoemd) in reactie op de aanwezigheid van buurplanten. De aanwezigheid van buurplanten wordt (deels) bepaald door de ratio tussen rood en ver-rood 
(R:FR) licht, en deze ratio induceert de twee plastische reacties. R:FR zal dalen in aanwezigheid van buurplanten omdat groene delen van de plant voornamelijk rood licht absorberen en ver-rood reflecteren. Dat de grootte en de vorm (fenotype) van de planten de R:FR ratio bepalen maar dat tegelijkertijd de R:FR ratio de fenotype van de planten beïnvloed geeft aan dat onderzoek naar plastische reacties in een dynamische omgeving complex kan zijn. In hoofdstuk 2 is daarom uiteengezet hoe 3D plant modellen gebruikt kunnen worden om schaduw ontwijkende reacties van het orgaan op te schalen naar de prestatie van de gehele plant in een vegetatie. Hierbij wordt geaccentueerd dat de dynamische cycli tussen plant experimenten en computer modeleren kan bijdragen aan het algeheel begrip van interacties tussen plant en omgeving.

Hoofdstuk 3 en 4, presenteren experimentele data en computersimulaties die laten zien dat variatie in plastische reacties invloed heeft op het concurrentievermogen van planten gedurende competitie voor licht. Hoofdstuk 3 presenteert het 3D Arabidopsis model waarin fenotypische plasticiteit is beschreven door een reactie functie die orgaan verandering relateert aan R:FR perceptie. Daarnaast bevat het experimentele data van variatie in petioolstrekking en competitie experimenten van verschillende Arabidopsis genotypen. Hoofdstuk 4 beschrijft plantexperimenten en computersimulaties die laten zien dat het induceren van de twee fenotypische plasticiteitreacties (petioolstrekking en hyponastie) afhankelijk zijn van de plaats op de bladeren waar R:FR wordt waargenomen. Er is geconcludeerd dat het concurrentievermogen versterkt als petioolstrekking wordt bepaald door R:FR perceptie op de petiool en hyponastie wordt bepaald door R:FR perceptie aan het uiteinde van het blad.

In hoofdstuk 5 is het 3D Arabidopsis model gebruikt om te bepalen of verschillende niveaus van plastische reacties geselecteerd kunnen worden in verschillende omgevingen gevormd door verschillende populatiedichtheden. Door principes van evolutionaire speltheorie toe te passen is geconcludeerd dat verschillende populatiedichtheden selecteren voor verschillende niveaus van plastische reacties. Echter, in de algemene discussie wordt een extra modelsimulatie gepresenteerd die deze conclusie aanvecht. Verder wordt in het laatste hoofdstuk benadrukt dat het combineren van experimenten, modellen en theorie essentieel is om interacties tussen plant en omgeving te begrijpen. 


\section{Acknowledgements}

In these last pages of my thesis I would like to thank everyone that was involved in and around my four-year PhD journey. Let's start thanking my three supervisors: Niels, Ronald and Jochem. I could not have hoped for a better team for this project; you are all great scientists, very open-minded to new ideas, you gave me the freedom to do my own thing, and you were always available for questions or just a chat! You all have your own expertise but contributed to the perspective of the other, which made the papers better. Jochem, I don't think there are many PhD students that would have a "daily supervisor" that they literally would see every day. Thank you so much for the carpool sessions; It was great being able to talk about work, but mainly talking about any other topic! It has been great working with you, your enthusiasm for FSP modelling is catchy and you are always open for discussion and new ideas. Ronald, your ability to understand and connect molecular, physiological and ecological plant research is really great. Thank you for inspiring me to combine all these aspects! Niels, your support in me and this project was really great, you have always been enthusiastic about all possibilities. Special thanks for the support in the last months when I decided to finish the thesis although time was not in my favour. It means a lot that you (also Jochem and Ronald) make time to respond fast to any question or text that I handed in. To all three of you, I truly hope that our paths will cross again someday!

Secondly, I want to thank my two great paranymphs, my two buddies in two groups: Emilie and Jorad. Emilie, although we like different work (pipetting vs modelling), we have worked together in different projects. More importantly, we became buddies at work and friends outside of work! I am very thankful for your kindness, patience and wisdom at work, but especially your friendship, talking and sharing about many things! Jorad, as some of our colleagues may think that our discussions are too intense, we know that this is our way of challenging each other about our work. I am impressed about your modelling skills, and your enthusiasm for difficult questions! But also thanks for joining me in coffee/lunch breaks outside to get some fresh air. Here I also want to mentioned my third paranymph-workbuddy Lot! Your enthusiasm, social skill and energy is really amazing! You are a true team- 
player as you always have time to help others. Thanks for your scientific questions, for just being a fun and great person and your great idea for the small detail in this thesis!

As I worked in two groups, there are a lot of people that I worked with or were involved in social interactions. From EVP I want to mention Chrysa; your commitment to your work is amazing and you managed our paper great, thank you so much for being my colleague! Jesse, it was great having you as MSc-student and I know that you will also do great with your own project. While working, keep up the fun and the social interactions, and the group is lucky with you! All the others from the "shade" group; Paulien, Debatosh, Chiakai, Sara, Sarah, Kasper, Scott, Diederik and all the students, thanks for always asking the right and challenging questions. I also want to thank Rens for hosting great BBQs, Rashmi for being critical but also fun in meetings or at coffee, Thijs for his knowledge, and Rob, Ankie, Judith, Marleen and Emilie for great experimental support, without you all the group would not produce anything! Maarten, thanks for your Italian influence and being my computer-scriptwrithing-nerd-friend at EVP. I also want to thank Fatima, Richard and Jojanneke, for the great deal of data collection. I have learned much from the supervision, and I had also great fun in these projects! Together with Hans, Elaine, Sjon, Shanice, Zeguang and Nikita I thank everyone from EVP for being great scientists and fun people at coffee, 'borrel' and dinners!

From CSA I especially want to thank Bob; I have come to you for many statistical questions and I have learned so much. However, your biological perspective is even a better contribution, thanks for that! Peter V. for all the great EGT explanations and discussions! All people from the "PFF"' group; Junqi, Martin, Marcello, Gu, Ningyi, Arian, Maarten, Rachel, Alejandro, David, loannis, and students. I always really loved our meetings, talking about modelling and experiments with so many different backgrounds always resulted in new insights and ideas! Also thanks for Paul, Xinyou, Tjeerd-Jan, Wopke, Lammert, Aad, Peter P., Willemien, Alex-Jan, Steven, Marjolein, Pepijn, Cor, Ans and Jet for any question at meetings or just good conversation at coffee. Big thanks for Sjanie and Nicole for their timeless help. Let's end this with also mentioning the other PhD students; Luuk, Ali, Kailei, Goufang, Yang, Wenjing, Uta, Niteen, and Dennis, for all the other fun interactions!

Next to the two groups in the Netherlands I had the privilege to go to Japan for two months. Therefore, I would like to thank Prof. Yoh Iwasa for his hospitality of welcoming me in his 
group in Fukuoka. Being in Japan was an experience on its own, but being in a Theoretical Biology group thinking about game theory, was also challenging. During my stay I have learned much and developed new ideas. I want to include the group members; dr. Satake, dr. Iwami, dr. Haeno, dr. Seki, dr. Tachiki, Yusuke, Ryo, Kubo, Douge-san, Satoi and of course Joung-Hun, Cynthia, Laura and Louise, for scientific discussions but also for taking me for lunch, dinner, karaoke, beers and tourist trips. Especially I want to thank Ryosuke for, among others, inviting me for his soccer-team! You really made me feel welcome in a new culture, city and country!

As many people have said in their acknowledgements; completing a PhD project does not only depend on colleagues, but also on friends and family. I want to thanks my friends and family for being interested (or just open) to listen to my nerdy talks about work. Having people to enjoy a drink and a chat with, will always be needed and keeps me being myself!

I especially want to thank my parents for being here, there, everywhere and always supporting me. Dad, your enthusiasm for science has sparked mine, and being able to share this with you is incredible. Maybe one day we will put Bongers and Bongers on a paper?! Mom, you have thought me that social interactions in and outside work are very important. The way you talk with strangers and let them feel welcome, but also the way you engage in the lives of your closed-ones, is amazing, and I hope to develop this social skill someday! At last I want to thank Florine, just for being yourself! Florine, you are great support; kind, patient, interested (although you understand half) and just as crazy as I am. Your passion to live abroad and experience other cultures is attractive, and I am looking forward to experience new adventures together, anywhere in the world. 



\section{Curriculum vitae}

Franca Bongers was born on the first of April, 1988 in Nijmegen, the Netherlands. After finishing high school in Nijmegen, she moved to Utrecht to study Biology. After finishing her bachelor Biology with special focus on plants, she joined the board of the Biology Student Association as president for a year. In 2011 she started the Master Environmental Biology, at Utrecht University, and she did three research internships. Firstly, she went to Cordoba, Spain, to do an ecological field study about the drought performance of Mediterranean species. Secondly, she stayed in Utrecht to perform a molecular - physiological study related to shade avoidance. Lastly, she went to Los Angeles, USA, to learn about leaf hydraulics and venation architecture. In 2013 she started her PhD research at the Centre for Crop Systems Analysis (Wageningen University) and Plant Ecophysiology (Utrecht University) groups. During her PhD project she also visited the Theoretical Biology group at Kyushu University in Japan. In her PhD research she studied how phenotypic plasticity, in the shape of shade avoidance responses, could affect plant competition for light and how variation in the degree of phenotypic plasticity could result from selection in different environments. For this study she combined physiological plant experiments, functional-structural plant models and evolutionary game theoretical principles. In August, 2017, Franca will move to Beijing, China, to do a postdoctoral research.

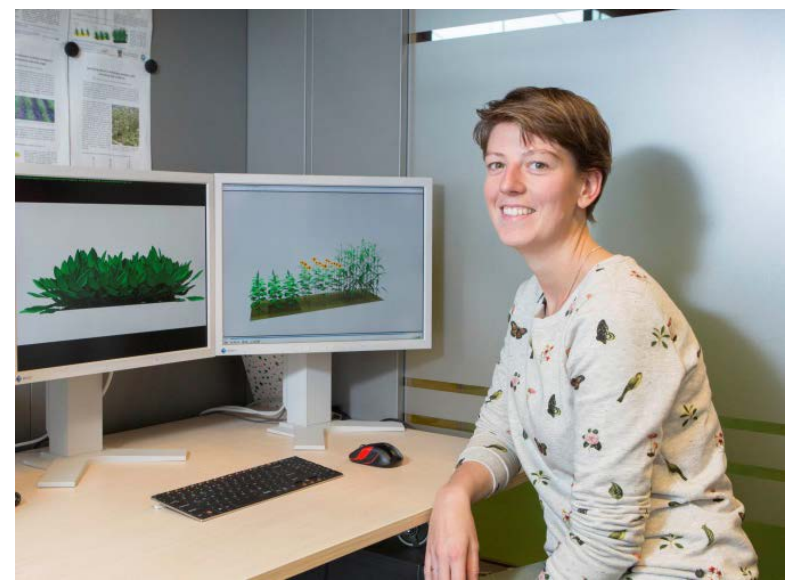





\section{Publications}

Bongers FJ, Evers JB, Anten NPR and Pierik R. (2014) From shade avoidance responses to plant performance at vegetation level: using virtual plant modelling as a tool. New Phytologist, 204, 268-272

Bongers FJ, Olmo M, Lopez-Iglesias B, Anten NPR and Villar R. (2017) Drought responses, phenotypic plasticity and survival of Mediterranean species in two different microclimatic sites. Plant Biology, 19, 386-395

Caringella MA, Bongers FJ and Sack L. (2015) Leaf hydraulic conductance varies with vein anatomy across Arabidopsis thaliana wilt-type and leaf vein mutants. Plant, Cell \& Environment, 38, 2735-2746

de Wit M, Keuskamp DH, Bongers FJ, Hornitschek P, Gommers CMM, Reinen E, MartínezCerón C, Fankhauser C and Pierik R. (2016) Integration of phytochrome and cryptochrome signals determines plant growth during competition for light. Current Biology, 26, 3320-3326 



\section{PE\&RC Training and Education Statement}

With the training and education activities listed below the PhD candidate has complied with the requirements set by the C.T. de Wit Graduate School for Production Ecology and Resource Conservation (PE\&RC) which comprises of a minimum total of 32 ECTS ( = 22 weeks of activities)

\section{Review of literature (4.5 ECTS)}

- From shade avoidance responses to plant performance at vegetation level: using virtual plant modelling as a tool

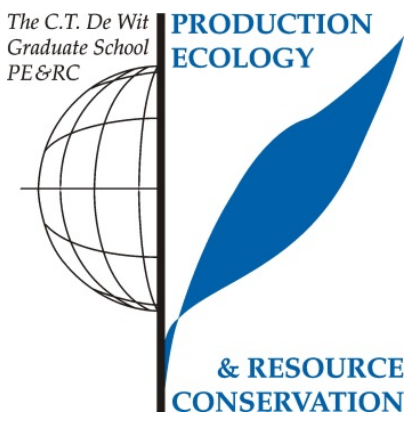

\section{Post-graduate courses (5.8 ECTS)}

- Photosynthesis, climate and change; PE\&RC (2013)

- Environmental Signalling; Summerschool UU(2013)

- Introduction to R statistics; PE\&RC (2014)

- $\quad$ PEPG Physiology field techniques; SEB \& BES (2016)

\section{Laboratory training and working visits (4.5 ECTS)}

- Evolutionary Game Theory; Kyushu University, Japan (2016)

I nvited review of (unpublished) journal manuscript (1 ECTS)

- New Phytologist : photosynthetic acclimation in wheat by 3D canopy reconstruction (2016)

\section{Competence strengthening / skills courses (3.1 ECTS)}

- PhD Competence assessment; WGS (2013)

- Communication with the media and the general public; PE\&RC (2014)

- Scientific writing; Wageningen in'to languages (2015)

PE\&RC Annual meetings, seminars and the PE\&RC weekend ( 3.4 ECTS)

- CoS-SAS Seminar day (2014)

- $\quad$ PE\&RC One-day symposium (2014, 2015)

- $\quad$ NERN Annual meeting (2014, 2015)

- WGS PHD Workshop carousel (2015)

\section{Discussion groups / local seminars / other scientific meetings (9.2 ECTS)}

- IEB PhD meetings; UU and GSLS PhD meetings; UU (2013-2015)

- Experimental Plant Sciences meeting; poster presentation; Lunteren $(2014,2015)$

- Plant Form and Function meetings (2014-2016)

- WEES Seminars (2015)

I nternational symposia, workshops and conferences (4.1 ECTS)

- GrolMP Workshop; Anger, France (2015)

- FSPMA; Qingdao, Chin (2016)

\section{Lecturing / supervision of practicals / tutorials (7.5 ECTS)}

- Plant adaptation and defence ; UU (2014)

- Ecology; WUR (2015)

- Functional diversity; WUR (2016)

\section{Supervision of MSc students}

- Shade avoidance responses curves affecting fitness in competition for light in difference Arabidopsis thaliana genotypes stands; UU

- Plastic responses to changing light quality in Arabidopsis thaliana; UU

- Leaf tip of Brassica nigra is most sensitive to low ratios of Red : Far-Red light; WUR

- $\quad$ Low R:FR induced change on biomass allocation; UU 
Funding: Netherlands Organisation for Scientific Research grant no. 821.01.014 Cover Design: UPPNA, Cassandra Rietbroek

Printing: GVO drukkers \& vormgevers B.V. 

